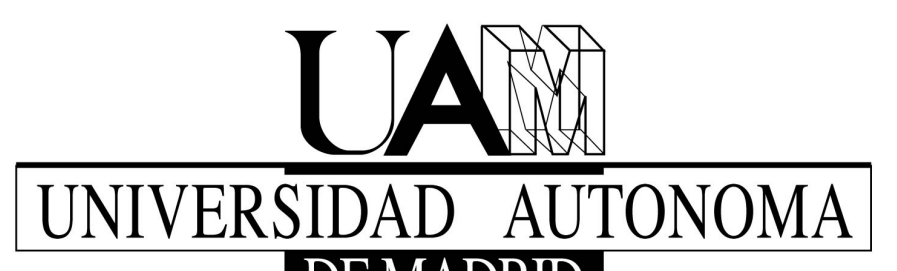

DEMADRID

Máster en Matemáticas y AplicaCiones

Trabajo Fin de Máster

\title{
NORM-ATTAINING OPERATORS
}

Ángela Capel Cuevas

AdVisors:

Dr. Miguel Martín SuÁrez

(Universidad de Granada)

Dr. José Pedro Moreno díaz

(Universidad Autónoma de Madrid)

SEPTEMBER 2015 

A los que ya no están, por todo lo que me han enseñado, y a los que aún siguen aquí, por lo que me ayudan día a día. 



\section{Contents}

1 INTRODUCTION.

1.1 PreVIOUS CONCEPTS

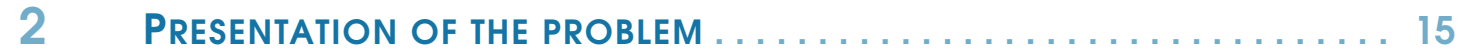

$\begin{array}{lll}2.1 & \text { SUPPORT MAPPINGS. BISHOP-PHELPS THEOREMS } & 16\end{array}$

2.2 Operators Which ATtAIN their NORM. THE LINDENStrauss-ZiZler THEOREM 20

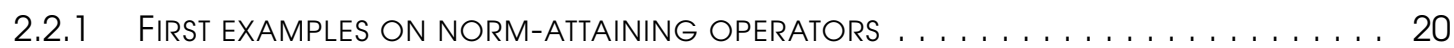

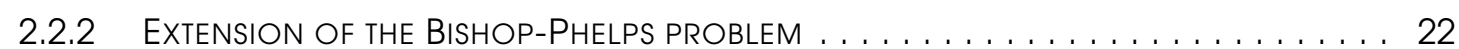

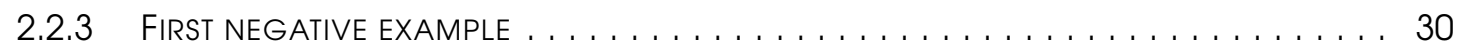

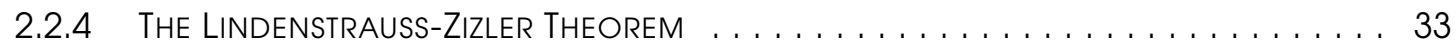

$\begin{array}{lll}2.3 & \text { SUMMARY } & 39\end{array}$

3 RESULTS ON NORM-ATTAINING OPERATORS ................ 43

3.1 Lindenstrauss PROPERTIES "A" AND "B" $4 \mathbf{4}$

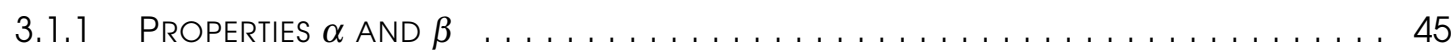

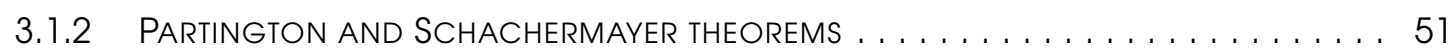

3.1.3 A SUFFICIENT CONDITION FOR RENORMING A BANACH SPACE WITH PROPERTY $\alpha \ldots 54$

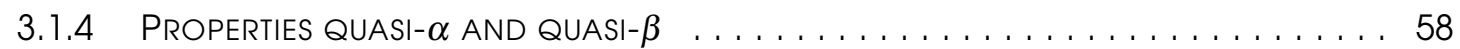

3.2 BISHOP-PHELPS AND RADON-NIKODYM PROPERTIES 63

3.2.1 THE NON-LINEAR OPtIMIZATION PRINCIPLE OF BOURGAIN AND STEGALL . . . . . . . . 69

3.2.2 Relation Between $\overline{N A(X)}=L(X)$ AND the RNP $\ldots \ldots \ldots \ldots \ldots \ldots \ldots \ldots$

3.3 DENSITY OF NORM-ATTAINING OPERATORS BETWEEN $C(K)$ AND $C(L) \quad 76$

3.3 .1 C $(K)$ SPACES WITH PROPERTY A $\ldots \ldots \ldots \ldots \ldots \ldots \ldots \ldots \ldots \ldots \ldots$

3.4 SUMMARY 83 
4 Counterexamples for $\overline{N A(X, Y)}=L(X, Y) \ldots \ldots \ldots \ldots . \ldots . \ldots$

4.1 COUNTEREXAMPLES BASED ON LINDENSTRAUSS' ARGUMENT 87

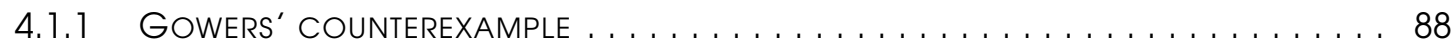

4.1.2 NO StRICTLY CONVEX INFINITE-DIMENSIONAL BANACH SPACE HAS PROPERTY B . . . . 90

$\mathbf{4 . 2}$ Other COUNTEREXAMPLES 93

$\begin{array}{lll}4.3 & \text { SUMMARY } & 97\end{array}$

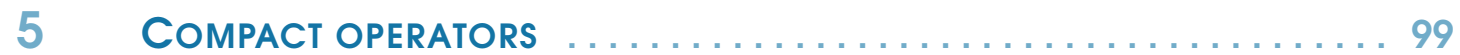

$\begin{array}{lll}5.1 & \text { The Approximation Property } & 100\end{array}$

$\begin{array}{lll}5.2 & \text { PROPERTIES } A^{k} \text { AND } B^{k} & 107\end{array}$

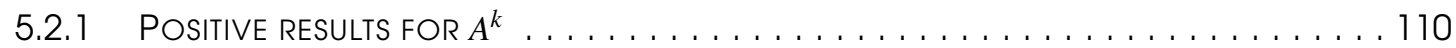

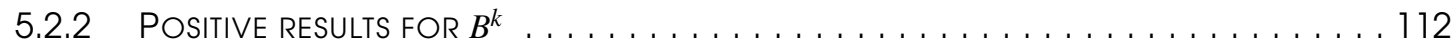

5.3 COMPACT NORM-ATTAINING OPERATORS AND THE DUNFORD-PETTIS PROPERTY 113

$\begin{array}{lll}5.4 & \text { SUMMARY } & 116\end{array}$

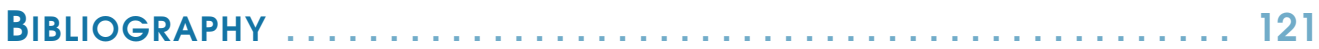

$\begin{array}{ll}\text { BOOKS } & 121\end{array}$

$\begin{array}{ll}\text { ARTICLES } & 122\end{array}$

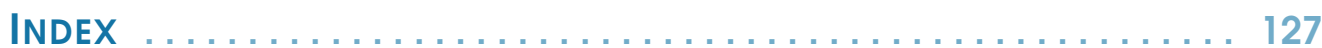




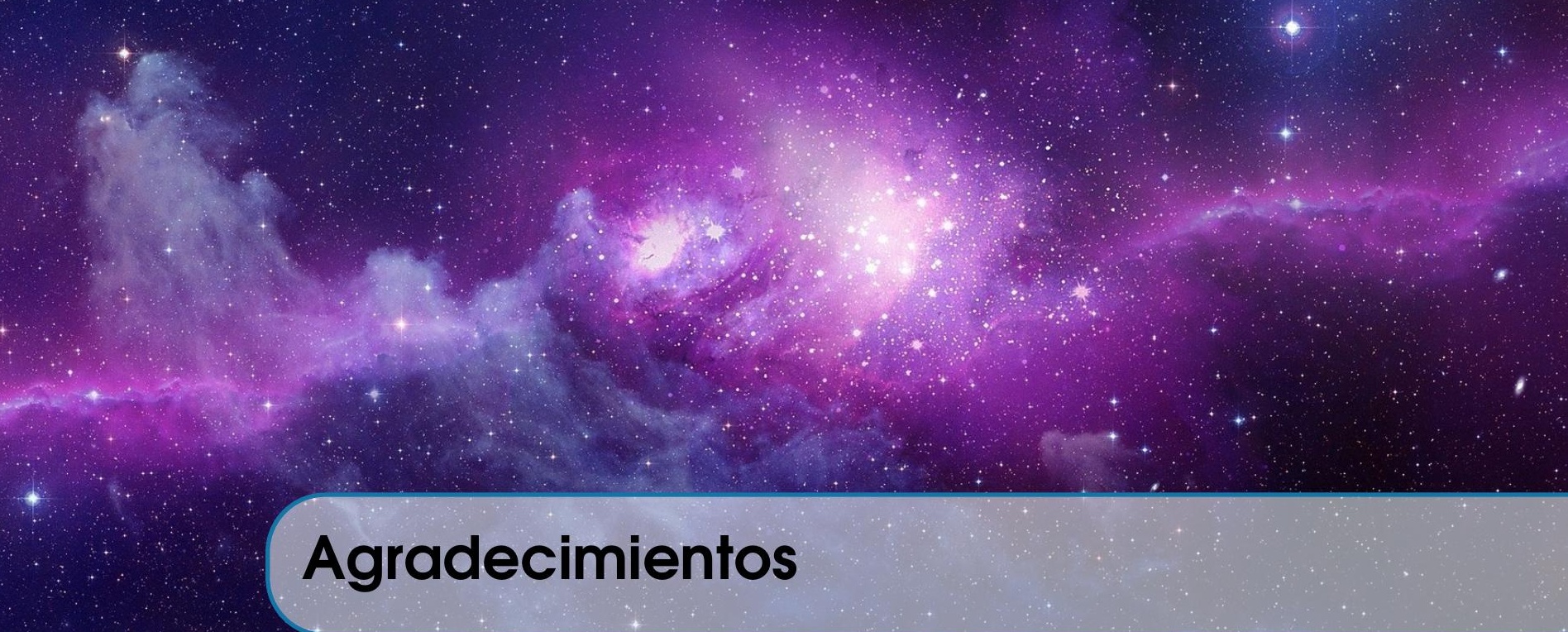

Quiero dedicar unas líneas para expresar mi agradecimiento a todas las personas que de un modo u otro han contribuido con su apoyo científico y/o personal a que este trabajo se haya podido realizar.

En primer lugar, quiero expresar mi más sincero agradecimiento a Miguel Martín, por haberme permitido iniciarme en el mundo de la investigación en un tema tan interesante, por todos los conocimientos y herramientas que me ha proporcionado y por la dedicación, energía y tiempo invertidos durante estos dos últimos años. Y también a José Pedro Moreno, por haber continuado la labor ya previamente comenzada, por sus constantes consejos y aportaciones a este trabajo, y por las interesantes charlas mantenidas en los últimos tiempos.

Quiero agradecer también al Departamento de Matemáticas de la Universidad Autónoma de Madrid, por su buena acogida durante este curso, y más concretamente a los profesores del Máster en Matemáticas y Aplicaciones, por el excelente trato que he recibido de cada uno de ellos y por todos los conocimientos aportados, además de numerosos sabios consejos. Y a todos mis compañeros de máster, por todo lo que me han aportado las numerosas conversaciones mantenidas durante las comidas, así como por su disponibilidad siempre para discutir algún problema.

Y, por supuesto, todo el trabajo realizado durante el máster no habría sido posible sin el conocimiento previamente adquirido durante la carrera, por lo que quiero agradecer a la Universidad de Granada y, más concretamente, a los profesores que han impartido las asignaturas que he cursado de las titulaciones de Licenciatura en Matemáticas y Grado en Física, así como a mis compañeros de ambas titulaciones, por todos los buenos momentos compartidos durante los años de la carrera, las fructíferas conversaciones mantenidas, el apoyo en la época de exámenes y las múltiples experiencias vividas.

No me puedo olvidar de la familia. Quiero agradecer a mis padres por darme la vida y por haber luchado en la suya día tras día para poder darme un buen futuro. La mayor parte de lo que soy hoy es gracias a vosotros y a vuestro constante apoyo. A mi hermano Ismael, por mostrarme desde pequeña que las matemáticas son divertidas y una gran opción de futuro, por todo lo que me ha enseñado en la vida y, cómo no, por cederme su casa. A mi hermana Sonia, por mostrarme cuál es el camino a seguir. A mis abuelos, por el cariño con el que siempre me trataron y lo 
mucho que aprendí de ellos, porque a veces el conocimiento más importante no está en los libros. $\mathrm{Y}$ al resto de mi familia y mis amigos, por el apoyo que siempre me han dado y la confianza que depositan en mí.

Y por último, pero no menos importante, quiero dar las gracias a Jesús, mi compañero de piso, de estudios, de fatigas, de vida. Gracias por cada uno de los minutos que has dedicado a ayudarme, no sólo en este trabajo, sino en todo lo demás. Gracias, porque no podría haber llegado hasta aquí sin tu ayuda, y por lo divertido que ha sido, después de tantos años compartiendo carrera y pasión, por fin ser compañeros de clase. En definitiva, gracias porque contigo a mi lado la vida es mucho más divertida e interesante. 


\section{INTRODUCTION}

This survey is presented as a 'Trabajo Fin de Máster' for the 'Máster en Matemáticas y Aplicaciones' of the 'Universidad Autónoma de Madrid'. The subject chosen for the survey is norm-attaining operators. The theory of norm-attaining operators is a very recent field of research, as it appeared in the second half of the 20th Century, and it is also very active nowadays, since many authors contribute to this field with their research now.

In spite of its short life, the work in the theory of norm-attaining operators has been very fruitful, and there exist many important results related to this field. However, these results are not completely collected in a monograph or anything similar, not even the most basic ones, what really complicates the initiation in the research in this field. For this reason, we have elaborated a survey about norm-attaining operators and the most important results about them which have been developped in the past years. We think that this survey may be used, in the future, for students who want to join the world of research in this field.

We have also worked in the specific case of endomorphisms. The problem of density of norm-attaining operators, as we will show later, involves two spaces, the spaces of the domain and codomain of the operators under study. Most of the results about this problem are for different spaces in domain and codomain. In this survey, we pay special attention to the case when domain and codomain are the same space. To distinguish the results about endomorphisms from the rest of the results, we will present them in green boxes.

The problem that we deal with in this survey has its origin in an important result of the theory of Banach spaces, the Bishop-Phelps Theorem. Although it appeared just half a century ago (more specifically, in 1961), it is considered a classical result of Functional Analysis. This theorem, as we will see at the beginning of the second chapter, states that the set of norm-attaining functionals for a Banach space is dense in the dual space.

From this theorem, a natural question comes out: If we consider the set of bounded linear operators between two Banach spaces $X$ and $Y(L(X, Y))$ and denote by $N A(X, Y)$ the set of norm-attaining operators between the same spaces, when is $N A(X, Y)$ norm-dense in $L(X, Y)$ ? Moreover, is the problem of the density true for all Banach spaces $X$ and $Y$ ? The answer to the second question is negative, as Lindenstrauss found a counterexample for the density of $N A(X, Y)$ in $L(X, Y)(\overline{N A(X, Y)} \neq L(X, Y))$ in 1963, and many other counterexamples have 
been developped later. However, although many authors have contributed to this problem and studied conditions for $X$ and $Y$ to satisfy $\overline{N A(X, Y)}=L(X, Y)$, after more than fifty years of research in this field, the first question asked above is very far from having a satisfactory answer.

One of the reasons of the importance of the problem of density of norm-attaining operators is its intimate conection with another important subject in Banach spaces, the Radon-Nikodym property. In fact, the study of norm-attaining operators provides certain familiarity with the geometric aspects of the RNP, like the dentability. It is also important because the Bishop-Phelps theorem constitutes the first 'perturbed optimization problem' in history and the result which can be considered the most important one in this theory is a non-linear optimization problem. These facts show the intimate relation of this field with many other fields of Mathematical Analysis, and, more specifically, Functional Analysis.

We have divided our survey into five chapters. The first chapter is introductory to the problem we are going to study later and contains the necessary basic concepts to understand the content of the following chapters.

In the second chapter, we will introduce the problem we are studying in this survey, the density of $N A(X, Y)$ in $L(X, Y)$ for all spaces $X$ and $Y$. We will begin with the simplest case, taking the range space as the field, i.e., the case of functionals, which are nothing but the elements of the dual space of the domain space. After introducing some results, we will see that the problem of density of $N A(X, \mathbb{K})$ in $L(X, \mathbb{K})$ (where $\mathbb{K}$ is the field) is completely solved, as we will show, in the important result mentioned above, the Bishop-Phelps Theorem, that this density holds for every Banach space $X$. This result appeared in 1961, although it was improved in 1963 by the same authors, with a generalization involving support functionals. In 1970, B. Bollobás made some quantitative aportations to this result, obtaining what we know nowadays as the Bishop-Phelps-Bollobás Theorem.

We will also mention that there exists a certain class of spaces, the reflexive spaces, such that every bounded linear functional in these spaces attains its norm. Moreover, this fact is a characterization of reflexivity. This result is the James Theorem and constitutes another classical result of Functional Analysis.

Next, once we know the result of the problem for functionals, we will extend the problem to the set of operators between arbitrary Banach spaces $X$ and $Y$ and study the density of $N A(X, Y)$ in $L(X, Y)$. First, we will consider the equality of these two sets of operators and study the conditions for $X$ and $Y$ to verify it, proving that a necessary condition for $X$ to verify it is to be reflexive, and proving also some conditions for $Y$. These examples will be, in particular, examples for the problem of the density.

Later, we will show the first counterexample for the problem of the density of norm-attaining operators, constructed by J. Lindenstrauss in 1963. We will also give a sketch of the procedure used to construct more counterexamples using the same method. The seminal paper of Lindenstrauss constitutes one of the biggest contributions to this field of research. He did not only show that the expression $\overline{N A(X, Y)}=L(X, Y)$ is not true in general, but also gave a slightly weaker affirmation which holds for every Banach spaces $X$ and $Y$. This result was improved by V. Zizler in 1973 and it provides, in particular, that the expression $\overline{N A(X, Y)}=L(X, Y)$ is true when $X$ is reflexive. In fact, from this result we obtain that reflexive spaces are positive examples for the problem of the density in the case of domain and codomain being the same space.

In the third chapter, we will continue with the study of properties and results about normattaining operators and the density of the set $N A(X, Y)$ in $L(X, Y)$ for certain Banach spaces $X$ and $Y$. We will begin with the definition of properties $A$ and $B$, defined by Lindenstrauss in the 
same paper mentioned above, and which have marked the development of the theory. A Banach space $X$ has property $A$ (resp. $B$ ) if the set of norm-attaining operators from $X$ to $Y$ (resp. from $Y$ to $X$ ) is dense in $L(X, Y)$ for every Banach space $Y$. Although these two properties are essential in the study of the problem of density of norm-attaining operators, we will notice that the problem of checking if a Banach space has one of them is really hard; this will lead to the appearance of properties $\alpha$ and $\beta$, geometric properties implying properties $A$ and $B$, respectively.

After presenting some spaces with properties $\alpha$ and $\beta$, we will prove that, indeed, $\beta$ implies $B$, and, with a slight modification, $\alpha$ implies $A$, but before that, we will have introduced the concept of uniformly strongly exposed points and proved that if the unit ball of a Banach space is the closed convex hull of a set of uniformly strongly exposed points, then the space has property A.

Properties $A$ and $B$ are not very restrictive from the isomorphic point of view. This is what J. R. Partington showed in 1982, when he proved that every Banach space can be renormed to satisfy property $\beta$ (therefore, with property $B$ ), and W. Schachermayer in 1983 , with the semi-analogous result for property $\alpha$, when he proved that every weakly compactly generated Banach space can be renormed with property $\alpha$. There is no complete duality between these two results, as there exist examples of Banach spaces that cannot be equivalently renormed to satisfy property $\alpha$. However, an improvement of Schachermayer Theorem appeared in 1993, by B. V. Godun and S. Troyanski, where they showed that even though not every Banach space can be equivalently renormed with property $\alpha$, there is a really big class of spaces that admit this renorming.

Other two geometric properties implying $A$ and $B$, respectively, are properties quasi- $\alpha$ and quasi- $\beta$. We will introduce property quasi- $\beta$, an intermediate property between $\beta$ and $B$, which is stable under $c_{0}$-sums, something that property $\beta$ does not satisfy. Analogously, we will present property quasi- $\alpha$, implying $A$ and being implied by $\alpha$, which is also stable by finite $\ell_{1}$-sums . These two properties have been presented and studied in recent works of M. D. Acosta, F. J. Aguirre and R. Payá (property quasi- $\beta$ ) and Y. S. Choi and H. G. Song (property quasi- $\alpha$ ).

In the next part of the same chapter, we reach what can be considered the most brilliant result in the theory of norm-attaining operators, the relation between the problem of density of normattaining operators and the Radon-Nikodym property, due to Bourgain, in 1977. He introduced a restrictive (and local) version of property $A$, which he called 'Bishop-Phelps property', and which is equivalent to the RNP. After presenting some definitions and important results, including Bourgain Theorem and the non-linear optimization principle of Bourgain and Stegall, we will conclude that a Banach space has the Radon-Nikodym property if, and only if, every space isomorphic to it has property $A$. This result is really important, as constitutes a link between the theory of norm-attaining operators and the field of measure theory; it is fundamental for the theory of norm-attaining operators, and at the same time constitutes a great advance in the comprehension of the geometric aspects of the RNP.

To continue with the relation between the theory of norm-attaining operators and the RadonNikodym property, we realise that, for a Banach space, the property that every space isomorphic to it has property $A$ is a very strong property, so we will study the possibility of weaken this property to a property that only involves the space of the domain and still has an equivalence with the RNP. Our conjecture is that, for a Banach space $X$, having the RNP is equivalent to verifying $\overline{N A(X)}=L(X)$ for every equivalent renorming of $X$. We will prove that if $X$ is isomorphic to a square of a Banach space, then this equivalence is true, using a very recent result of M. Martín, and provide some positive and negative examples of spaces that are isomorphic to a square of a Banach space. The extension of the proof of the previous equivalence is still an open problem. 
To finish the third chapter, we will study the specific case of the density of norm attaining operators between two spaces of the type $C(K)$. For that, we will present some results to show that if $K, L$ are compact, Hausdorff topological spaces, then the set of norm-attaining operators between $C(K)$ and $C(L)$ is dense in the set of bounded linear ones. Later, we will see that $C(K)$ has property $A$ if, and only if, $K$ is finite, and we will also present some results of density for the set $N A(C(K), Y)$ when there exists a weakly compact operator from $C(K)$ to $Y$.

In the fourth chapter, we will present more counterexamples for the problem of density of norm-attaining operators. We have mentioned above that in the second chapter we will present the first counterexample for this problem, Lindenstrauss' counterexample. In the first part of this chapter, we will use the same arguments used by him to construct some new counterexamples.

In 1990, W. Gowers constructed a space (nowadays called Gowers' space after him), and proved that if we consider a strictly convex Banach space $Y$ such that there exists a non-compact operator from Gowers' space into $Y$, then the couple $(G, Y)$ constitutes a counterexample for the problem of density. With a slight modification of the techniques used by Gowers, we see in this chapter that if a strictly convex space contains a subspace which is isomorphic to $\ell_{p}$ for any $1<p<\infty$, then $Y$ does not have property $B$.

Later, we will show another counterexample based on Lindenstrauss' argument, which constitutes a generalization of the counterexamples of Lindenstrauss and Gowers. In this result, M. D. Acosta showed that no strictly convex infinite-dimensional Banach space has property $B$, what provides new examples of classical Banach spaces without property $B$.

In the last part of this chapter, we will present other counterexamples which are not based on Lindenstrauss' argument. We will discuss an example constructed by Johnson and Wolfe of a compact, metric space $K$ such that $\overline{N A\left(L^{1}[0,1], C(K)\right)} \neq L\left(L^{1}[0,1], C(K)\right)$. This counterexample is inspired on another one of Schachermayer, for the couple $\left(L^{1}[0,1], C[0,1]\right)$, which is more extense and complicated.

Finally, in the last chapter, we will study the set of compact norm-attaining operators between two Banach spaces and its density in the set of compact operators between those two spaces. This problem has its origin in the problem of density of the set of norm-attaining operators between two Banach spaces, although it constitutes an interesting field of study itself. In the counterexamples shown in chapters two and four, we can see that the essential point in their construction is the fact that the closure of the set of norm-attaining operators is contained in the set of compact operators, so if there exists a non-compact operator, it cannot be approximated by norm-attaining operators. Therefore, a natural question to ask is whether every compact operator between two Banach spaces can be approximated by norm-attaining operators. In the past year, this question was answered negatively by M. Martín. To show that, we will first introduce the approximation property and some properties and results about it. We will show many equivalent formulations of this property and use them to prove some results on norm-attaining compact operators.

Later, we will define properties $A^{k}$ and $B^{k}$ for norm-attaining compact operators analogously as we did when we introduced properties $A$ and $B$ for norm-attaining operators, and show positive and negative examples of spaces verifying these properties. We will see that although many of these examples appear when we study properties $A$ and $B$ (even though we do not know whether properties $A$ and $B$ imply $A^{k}$ and $B^{k}$, respectively), there exist several results which are specific for properties $A^{k}$ or $B^{k}$ and not related to properties $A$ or $B$, respectively. Then, we will finish the survey introducing the Dunford-Pettis property and exposing some results relating this property with the density of norm-attaining compact operators. 


\subsection{PreVIOUS CONCEPTS}

In this section, we will introduce some concepts and notation which are necessary for the following chapters.

Let $X$ be a normed space. We say that $X$ is a Banach space if it is complete, i.e., if every Cauchy sequence in $X$ converges in $X$.

If $X$ and $Y$ are normed spaces over the same ground field $\mathbb{K}$, the set of all continuous $\mathbb{K}$-linear maps $T: X \rightarrow Y$ (which we call bounded linear operators, or just operators in the following chapters, as we will only work with this class of operators) is denoted by $L(X, Y)$. In infinite dimensional spaces, not all linear operators are continuous. A linear operator from a normed space $X$ to another normed space is continuous if, and only if, it is bounded on the closed unit ball of $X$. Therefore, the vector space $L(X, Y)$ can be given the operator norm

$$
\|T\|=\sup \left\{\|T x\|_{Y} \mid x \in \mathbb{B}_{X}\right\},
$$

where we denote by $\mathbb{B}_{X}$ the closed unit ball of $X$, i.e., the elements $x \in X$ such that $\|x\|_{X} \leq 1$.

We say that an operator attains its norm (or it is a norm-attaining operator) if the supremum in the previous definition is a maximum, i.e., if there exists $x_{0} \in \mathbb{B}_{X}$ such that $\|T\|=\left\|T x_{0}\right\|_{Y}$. We will denote the set of norm-attaining operators between two Banach spaces $X$ and $Y$ by $N A(X, Y)$.

If $X$ and $Y$ are normed spaces, they are isomorphic if there exists a linear bijection $T: X \rightarrow Y$ such that $T$ and its inverse $T^{-1}$ are continuous. If one of the two spaces $X$ or $Y$ is complete (or reflexive, separable, etc) then so is the other space. Two normed spaces $X$ and $Y$ are isometrically isomorphic if, in addition, $T$ is an isometry, i.e., $\|T x\|=\|x\|$ for every $x$ in $X$.

We will introduce now some classical Banach spaces, which we will mention during the text. These spaces include the $L^{p}$ spaces and their special cases, the sequence spaces $\left(\ell_{p}\right.$, for $1 \leq p<\infty, c, c_{0}$ and $\ell_{\infty}$ ), and the space $C(K)$ of continuous scalar function on a compact Hausdorff space $K$.

$\ell_{p}$ spaces

For every $1 \leq p<\infty$, we define $\ell_{p}$ as the subspace of $\mathbb{K}^{\mathbb{N}}$ consisting of all sequences $x=\left\{x_{n}\right\}$ satisfying

$$
\sum_{n}\left|x_{n}\right|^{p}<\infty
$$

Then, the real valued operation $\|\cdot\|_{p}$ defined by

$$
\|x\|_{p}=\left(\sum_{n}\left|x_{n}\right|^{p}\right)^{1 / p}
$$

defines a norm on $\ell_{p}$. As $\ell_{p}$ is a complete metric space with respect to this norm, it is a Banach space.

\section{$\ell_{\infty}$ space}

We define $\ell_{\infty}$ to be the space of all bounded sequences. A sequence $x=\left\{x_{n}\right\}$ belongs to $\ell_{\infty}$ if

$$
\sup _{n}\left|x_{n}\right|<\infty .
$$


Then, $\ell_{\infty}$ with the norm

$$
\|x\|_{\infty}=\sup _{n}\left|x_{n}\right|
$$

is a Banach space.

$c$ and $c_{0}$

We define $c$ as the space of convergent sequences, i.e., $x \in \mathbb{K}^{\mathbb{N}}$ belongs to $c$ if $\lim _{n \rightarrow \infty} x_{n}$ exists. Since every convergent sequence is bounded, $c$ is a linear subspace of $\ell_{\infty}$. It is, moreover, a closed subspace with respect to the infinity norm, and so also a Banach space.

The subspace of null sequences $c_{0}$ consists of all sequences whose limit is zero. This is a closed subspace of $c$, and so again a Banach space.

\section{$L^{p}$ spaces}

An $L^{p}$ space may be defined as a space of functions for which the $p$-th power of the absolute value is Lebesgue integrable. More generally, let $1 \leq p<\infty$ and $(S, \Sigma, \mu)$ be a measure space. Consider the set of all measurable functions from $S$ to $\mathbb{C}$ or $\mathbb{R}$ whose absolute value raised to the $p$-th power has finite integral, or equivalently, that

$$
\|f\|_{p}=\left(\int_{S}|f|^{p} d \mu\right)^{1 / p}<\infty .
$$

The set of such functions forms a vector space with the following operations

$$
\begin{gathered}
(f+g)(x)=f(x)+g(x), \\
(\lambda f)(x)=\lambda f(x)
\end{gathered}
$$

for every scalar $\lambda$. This set, with the function $\|\cdot\|_{p}$ is a seminorm. If we take the quotient of this space over the set of functions which are null $\mu$-almost everywhere, we obtain a Banach space, which we call $L^{p}(S, \mu)$.

For $p=\infty$, we define $L^{\infty}(S, \mu)$ as the set of all measurable functions from $S$ to $\mathbb{C}$ or $\mathbb{R}$ which are bounded (two functions are identified if they are equal $\mu$-almost everywhere).

\section{$C(K)$ spaces}

Let $K$ be a compact Hausdorff space. The space $C(K)$ is the space of continuous functions on $K$ with values in the real or complex numbers. This space is a vector space with respect to the pointwise addition of functions and scalar multiplication by constants. It is a Banach space with respect to the norm

$$
\|f\|=\sup _{x \in X}|f(x)|
$$


SUPPORT MAPPINGS. BISHOP-PHELPS THEOREMS

OPERATORS WHICH ATTAIN THEIR NORM. THE

LINDENSTRAUSS-ZIZLER THEOREM

FIRST EXAMPLES ON NORM-ATTAINING OPERATORS

EXTENSION OF THE BISHOP-PHELPS PROBLEM FIRST NEGATIVE EXAMPLE

THE LINDENSTRAUSS-ZIZLER THEOREM

SUMMARY

\section{Presentation of the problem}

In this chapter, we will introduce the problem we are studying in this survey, the density of the set of norm-attaining operators between two Banach spaces in the set of bounded linear operators between those spaces.

The notation we will use in general is $X$ for the domain space and $Y$ for the codomain. We will begin with the simplest case, taking $Y$ as the field. In this case, we will study the particular case of operators known as functionals, which are nothing but the elements of the dual space of $X$. After introducing some results, we will see that the problem of density of the set of norm-attaining functionals in the set of bounded linear ones is completely solved, as we will show, in an important result known as the Bishop-Phelps Theorem, that this density holds for every Banach space $X$.

In fact, we will show that exists a certain class of spaces, the reflexive spaces, such that every bounded linear functional in these spaces attains its norm. Moreover, this fact is a characterization of reflexivity.

Next, once we know the result of the problem for functionals, we will extend the problem to the set of operators between arbitrary Banach spaces $X$ and $Y$. For that, we will define the set of norm-attaining operators between two Banach spaces $X$ and $Y$ and study the density its density in the set of bounded linear operators between those two spaces.

We will begin studying the equality of these two sets of operators, proving that a necessary condition for $X$ to verify it is to be reflexive, and proving also some conditions for $Y$. These examples will be, in particular, examples for the problem of the density.

Next, we will show the first counterexample for the problem of the density of norm-attaining operators, constructed by J. Lindenstrauss in 1963. We will also give a sketch of the procedure used to construct more counterexamples using the same method.

Finally, at the end of the chapter, we will enunciate and demonstrate the Lindenstrauss-Zizler theorem, which shows that the set of the operators from $X$ to $Y$ whose adjoints attain their norm is dense in the set of bounded linear ones. From this result we will obtain, in particular, that every reflexive space verifies the problem of the density in the case of domain and codomain being the same space. 


\section{1 SUPPORT MAPPINGS. BISHOP-PHELPS THEOREMS}

Let $X$ be a normed space and $f$ a bounded linear functional (from now on, just functional). Then, we define the norm of $f$ by

$$
\|f\|=\sup \left\{|f(x)|: x \in \mathbb{B}_{X}\right\},
$$

where $\mathbb{B}_{X}$ is the closed unit ball of $X$.

Definition 2.1.1 - NORM-ATtaINING FUNCTIONAL.

We say that a functional $f$ attains ist norm when the supremum in the previous definition is a maximum, i.e., if there exists $x_{0} \in \mathbb{B}_{X}$ such that $\|f\|=\left|f\left(x_{0}\right)\right|$.

Let us show some examples of norm-attaining functionals in $\ell_{1}^{*}$ and $c_{0}^{*}$, respectively.

- Example 2.1.2 In $\ell_{1}:$ Consider the functional defined by

$$
\begin{array}{cccc}
f: \quad \begin{array}{c}
\ell_{1} \\
x=\left\{x_{n}\right\}
\end{array} & \mapsto \quad \mathbb{K} \\
& \mapsto(x)=\sum_{k=1}^{\infty} \frac{x_{k}}{k} .
\end{array}
$$

Then, it is a bounded linear functional, as for every $x \in \ell_{1}$

$$
|f(x)| \leq \sum_{k=1}^{\infty}\left|\frac{x_{k}}{k}\right| \leq \sum_{k=1}^{\infty}\left|x_{k}\right|<\infty \quad\left(x \in l_{1}\right) .
$$

And if $x \in \mathbb{B}_{l_{1}},|f(x)| \leq 1$, so $\|f\| \leq 1$.

Now, if we take $x=(1,0, \ldots)$, it is clear that $\|x\|_{1}=1$ and $f(x)=1=\|f\|$. Thus, $f$ attains its norm.

In $c_{0}$ : Consider the functional defined by

$$
g: \begin{array}{ccc}
c_{0} & \longrightarrow & \mathbb{K} \\
x=\left\{x_{n}\right\} & \mapsto & g(x)=x_{1}+x_{2} .
\end{array}
$$

This functional is clearly linear and bounded, as for every $x \in c_{0}$ we have $\|x\|_{\infty}<\infty$, so $|g(x)| \leq 2\|x\|_{\infty}<\infty$, and $\|g\| \leq 2$.

And if we take $x=(1,1,0, \ldots) \in c_{0}$, we have $\|x\|_{\infty}=1$ and $g(x)=|1+1|=2$, so $g$ attains its norm.

We want to study the existence of this kind of functionals for every Banach space $X$. For that, we can begin using the Hahn-Banach theorem:

\section{Theorem 2.1.3 - HAHN-BANACH THEOREM.}

Let $X$ be a normed space over $\mathbb{K}$ and $M$ a proper subspace. Let $g: M \rightarrow \mathbb{K}$ be continuous and linear. Then, there exists an extension $f: X \rightarrow \mathbb{K}$ of $g$, which is also linear and continuous, such that $\|f\|=\|g\|$.

Then, as a consequence of this theorem, we have the following corollary: 
Corollary 2.1.4 For every $x \in X$, there exists $f \in X^{*}$ verifying $\|f\|=1$ and $f(x)=\|x\|$.

Using this corollary in the particular case of the sphere, given $x_{0} \in \mathbb{S}_{X}$ there exists $f \in X^{*}$ with $\|f\|=1$ and $f\left(x_{0}\right)=\left\|x_{0}\right\|=1$. Consequently, for every Banach space there exist functionals which attain their norm. Moreover, as a consequence of the Hahn-Banach theorem, it is easy to prove that the subspace of $X^{*}$ generated by the functionals which attain their norm is dense for the $\omega^{*}$-topology.

Once we have seen this property, a natural question to ask is, given a Banach space $X$, how many functionals in $X$ attain their norm, or even if there exists a Banach space where every functional attains its norm. We will show that for every Banach space $X$ the set of norm-attaining functionals is quite big, as it is dense in the set of bounded linear functionals, and, in fact, they are sometimes the same set, for reflexive spaces.

However, we can show that, in general, there exist non-norm-attaining functionals (except if the space is reflexive). We will show now some examples of functionals of this kind in the same spaces we used before, $l_{1}$ and $c_{0}$ (we choose these spaces as they are some of the most-known classical Banach spaces and are not reflexive).

- Example 2.1.5 In $\ell_{1}:$ Consider the functional defined by

$$
\begin{array}{ccc}
f: \quad \begin{array}{c}
\ell_{1} \\
x=\left\{x_{n}\right\}
\end{array} & \mapsto \quad \mathbb{K} \\
& \mapsto f(x)=\sum_{k=1}^{\infty}\left(1-\frac{1}{k}\right) x_{k} .
\end{array}
$$

It is a bounded linear functional, as for every $x \in \ell_{1}$

$$
|f(x)| \leq \sum_{k=1}^{\infty}\left|\left(1-\frac{1}{k}\right) x_{k}\right| \leq \sum_{k=1}^{\infty}\left|x_{k}\right|<\infty \quad\left(x \in \ell_{1}\right) .
$$

Then, $\|f\| \leq 1$. If we consider $e_{n}=(0, \ldots, 0,1,0, \ldots) \in \ell_{1}$, we have $\left\|e_{n}\right\|=1$ for every $n \in \mathbb{N}$ and

$$
\left|f\left(e_{n}\right)\right|=\left|1-\frac{1}{n}\right| \text { and } \lim _{n \rightarrow \infty}\left|1-\frac{1}{n}\right|=1
$$

Therefore,

$$
\lim _{n \rightarrow \infty}\left|f\left(e_{n}\right)\right|=1 \Rightarrow\|f\|=1
$$

However, if $x \in \mathbb{B}_{\ell_{1}}$,

$$
|f(x)| \leq \sum_{k=1}^{\infty}\left|\left(1-\frac{1}{k}\right) x_{k}\right|<\sum_{k=1}^{\infty}\left|x_{k}\right|=\|x\|_{1},
$$

as $\left|1-\frac{1}{k}\right|<1$ for every $k \in \mathbb{N}$, and there is at least one $x_{k} \neq 0$.

Then, $f$ does not attain its norm.

In $c_{0}$ : Consider the functional defined by 


$$
\begin{array}{rlll}
g: & c_{0} & \longrightarrow & \mathbb{K} \\
x=\left\{x_{n}\right\} & \mapsto g(x)=\sum_{k=1}^{\infty} 2^{-k+1} x_{k} .
\end{array}
$$

Then, it is linear and bounded:

$$
|g(x)| \leq \sum_{k=1}^{\infty}\left|2^{-k+1}\right|\left|x_{k}\right| \leq \sum_{k=1}^{\infty} 2^{-k+1}=2
$$

So $\|g\| \leq 2$. Now, taking $v_{n}=(1,1, \ldots, \stackrel{(n)}{1}, 0 \ldots) \in c_{0} \Rightarrow\left\|v_{n}\right\|=1$ and

$$
\left|g\left(v_{n}\right)\right|=\left|\sum_{k=1}^{n} 2^{-k+1}\right|=2\left(1-\frac{2}{2^{n}}\right) \stackrel{n \rightarrow \infty}{\longrightarrow} 2 .
$$

Then, $\|g\|=2$. For proving that $g$ does not attain its norm, we observe that if $x \in c_{0}$, then there exists $k_{0} \in \mathbb{N}$ such that if $k \geq k_{0} \Rightarrow\left|x_{k}\right|<\frac{1}{2}<1$.

Hence, if $x \in \mathbb{B}_{c_{0}}$,

$$
|f(x)| \leq \sum_{k=1}^{\infty}\left|2^{-k+1}\right|\left|x_{k}\right|=\sum_{k=1}^{k_{0}}\left|2^{-k+1}\right|\left|x_{k}\right|+\sum_{k=k_{0}}^{\infty}\left|2^{-k+1}\right|\left|x_{k}\right|<\sum_{k=1}^{\infty} 2^{-k+1}=2
$$

where in the last inequality we have used $\sum_{k=k_{0}}^{\infty} 2^{-k+1}\left|x_{k}\right|<\sum_{k=k_{0}}^{\infty} 2^{-k+1}$, as $\left|x_{k}\right|<1 \forall k>k_{0}$.

We say that a Banach space is reflexive if it coincides with its bidual space. More specifically, if we denote the dual space of $X$ by $X^{*}$, and the bidual space by $X^{* *}$, and consider for every $x \in X$ the function $J(x): X^{*} \rightarrow \mathbb{K}$ given by

$$
J(x)(f)=f(x) \quad f \in X^{*},
$$

then $J(x) \in X^{* *}$, so we obtain a map $J: X \rightarrow X^{* *}$ called the evaluation map. From the HahnBanach theorem, $J$ is injective and preserves norms. A Banach space is reflexive when its evaluation map is surjective.

If $X$ is a reflexive Banach space, every functional $f \in X^{*}$ attains its norm, something that we can see as a consequence of Lemma 2.2.5, as $\mathbb{B}_{X}$ is compact with the weak topology, a functional in $X^{*}$ is continuous from $X$ to $\mathbb{K}$ with their respective weak topologies, and the weak topology of $\mathbb{K}$ coincides with the topology of the norm because $\mathbb{K}$ is finite dimensional.

Now, the reciprocal is given by the following theorem:

\section{Theorem 2.1.6 - JAMES THEOREM.}

A Banach space $X$ is reflexive if, and only if, every continuous linear functional on $X$ attains its maximum on the closed unit ball in $X$.

In the previous pages, we have studied the existence of norm-attaining functionals given a Banach space $X$. We have seen that, for every Banach space, there exist norm-attaining functionals, and that for some spaces, reflexive spaces, every functional attains its norm. An intermediate situation appears when the functionals which attain their norm are a dense set in 
$X^{*}$ for the topology of the norm. It is a natural question to ask for which Banach spaces this happens.

For some time, Banach spaces with that condition, the density of the set of norm-attaining functionals in $X^{*}$ for the topology of the norm, were called subreflexive. However, in 1961, E.Bishop and R.Phelps showed in [BP61] that every Banach space is subreflexive. Moreover, two years later, they published a paper [BP63] where they showed a better result, which is known as the Bishop-Phelps theorem. Before enunciating these results, we need some previous definitions.

\section{Definition 2.1.7 - SUPPORT MAPPING.}

Let $B$ be a convex, closed subset of a topological vector space $X$. If $x_{0} \in B$ and $f \in X^{*}$ verify

$$
\operatorname{Re} f(x) \leq \operatorname{Re} f\left(x_{0}\right) \quad \forall x \in B
$$

we say that $f$ supports $B$ at a point $x_{0}$, or, equivalently, that $x_{0}$ is a support point and $f$ a support mapping of $B$.

If $X$ is a normed space and $f \in X^{*}$ attains its norm, there exists $x_{0} \in \mathbb{B}_{X}$ such that $f\left(x_{0}\right)=\|f\|$. Also,

$$
\operatorname{Re} f(x) \leq\|f\| \quad \forall x \in \mathbb{B}_{X}
$$

Thus, Re $f$ attains its maximum in $\mathbb{B}_{X}$ and $f$ is a support mapping of $\mathbb{B}_{X}$.

With these definitions, we can now enunciate the main result of this section:

\section{Theorem 2.1.8 - BISHOP-PHELPS THEOREM.}

Let $B$ be a convex, closed subset of a Banach space $X$. Then:

- The set of support points of $B$ is dense in the boundary of $B$.

- The set of support functionals of $B$ is norm-dense in the cone of all continuous, linear functionals with bounded from above real part.

The proof of this result appears in [BP63].

In the real case, we can prove the following result, which is quantitatively better than the second part of the previous theorem:

Theorem 2.1.9 Let $C$ be a convex, closed subset of a Banach space $X$, and $f \in \mathbb{S}_{X^{*}}$ a functional whose real part is bounded from above in $C$. Let $a$ be a point of $C$ and $0<\varepsilon<1$, such that

$$
\operatorname{Re} f(a)>\sup \{\operatorname{Re} f(x): x \in C\}-\frac{\varepsilon^{2}}{3}
$$

Then, there exists a functional $g \in \mathbb{S}_{X^{*}}$ that supports $C$ at a point $b$, verifying

$$
\|b-a\|<\varepsilon \quad \text { and } \quad\|g-f\|<\varepsilon .
$$

Now, if we consider the particular case for $C$ of the unit ball, we obtain the modification of the Bishop-Phelps theorem into the Bishop-Phelps-Bollobàs [Bol70] one: 


\section{Corollary 2.1.10 - BISHOP-PHELPS-BOLLOBÀS THEOREM.}

Let $X$ be a Banach space. Let $x \in \mathbb{S}_{X}, f \in \mathbb{S}_{X^{*}}$ be such that

$$
\operatorname{Re} f(x)>1-\frac{\varepsilon^{2}}{3}
$$

with $0<\varepsilon<1$. Then, there exist $y \in \mathbb{S}_{X}, g \in \mathbb{S}_{X^{*}}$, verifying

$$
g(y)=1, \quad\|x-y\|<\varepsilon \quad \text { and } \quad\|g-f\|<\varepsilon .
$$

To sum up, we have seen in this section that every Banach space is subreflexive, i.e., for every Banach space the set of norm-attaining functionals is dense in the dual space for the topology of the norm. In fact, we have also seen that this condition can be improved as an equality of the sets when the space is reflexive (and only in that case).

Now, in the following section, we will extend the definition of norm-attaining functional to norm-attaining operator and study the same problem for an arbitrary Banach space in the codomain, instead of the field. We will see that the answer of that problem is not so easy as in this case, and that there are still many open questions for certain Banach spaces in the domain and codomain.

\subsection{OPERATORS WHICH ATtAIN THEIR NORM. THE LINDENSTRAUSS-ZizLER THEOREM}

In this section, we extend the concept of norm-attaining functional to norm-attaining operator, and try to extend the result of Bishop and Phelps to this kind of operators between two Banach spaces.

\subsection{FIRST EXAMPLES ON NORM-ATTAINING OPERATORS}

\section{Definition 2.2.1 - NORM-ATTAINING OPERATOR.}

Let $X$ and $Y$ be two Banach spaces and $T \in L(X, Y)$ an operator (and by that we mean a "bounded linear operator"). We say that $T$ attains its norm if there exists $x_{0} \in \mathbb{S}_{X}$ such that

$$
\left\|T x_{0}\right\|=\|T\|,
$$

i.e., if the supremum in the definition of $\|T\|$ is a maximum.

In the previous section, we saw that for every Banach space there exist norm-attaining functionals in its dual space, as a consequence of the Hahn-Banach theorem. This result can be considered the first natural example of norm-attaining operators. However, as the Hahn-Banach theorem is only valid with the field as the codomain, it is clear that we cannot use it now to guarantee the existence of norm-attaining operators.

In fact, it remains an open question if for every $X$ and $Y$ Banach spaces there exists a normattaining operator from $X$ to $Y$ (a non semi-trivial one). Therefore, the first step of the previous section cannot be extended to an arbitrary $Y$. However, we can study the density of the set of norm-attaining operators in $L(X, Y)$.

Before studying the density of these operators, we can show some examples of specific operators which attain their norm and others that do not.

- Example 2.2.2 For the first example, we want to show an operator that does not attain its maximum in the unit ball. 
We consider $c=\left\{c_{n}\right\} \in \ell_{\infty}$ such that $\left|c_{n}\right|<\sup \left|c_{n}\right|$ (for example, take $c_{n}$ positive and growing to 1 ) and the operator $T: \ell_{2} \rightarrow \ell_{2}$ given by

$$
T(x)=c x=\left\{c_{n} x_{n}\right\}, \quad \forall x=\left\{x_{n}\right\} \in \ell_{2} .
$$

Then, for any $x=\left\{x_{n}\right\} \in \ell_{2}$, we have

$$
\|T x\|^{2}=\sum_{n \in \mathbb{N}}\left|c_{n} x_{n}\right|^{2}<\sum_{n \in \mathbb{N}}\left(\sup \left|c_{n}\right|^{2}\right)\left|x_{n}\right|^{2}=\left(\sup \left|c_{n}\right|^{2}\right)\|x\|^{2} .
$$

Therefore, $\|T\| \leq \sup \left|c_{n}\right|$. On the other hand, if we choose $x=e_{n}=\left\{\delta_{k, n}\right\}_{k \in \mathbb{N}}$, where $\delta_{k, n}$ is the Kronecker- $\delta$, we have

$$
\sup _{\|x\| \leq 1}\|T x\| \geq \sup _{n \in \mathbb{N}}\left\|T e_{n}\right\|=\sup _{n \in \mathbb{N}}\left|c_{n}\right| .
$$

Hence,

$$
\|T\|=\sup \left|c_{n}\right|,
$$

and as $\|T x\|<\left(\sup \left|c_{n}\right|\right)\|x\|$ for every $x \in \ell_{2}, T$ does not attain its norm.

- Example 2.2.3 In this example, we will study the Fourier coefficients associated to a function. For that, we define,

$$
\begin{array}{ccc}
T: L^{1}(\mathbb{T}) & \longrightarrow & c_{0} \\
f & \mapsto & \{\hat{f}(n)\}
\end{array}
$$

where $\{\hat{f}(n)\}$ is the sequence of Fourier coefficients associated to $f \in L^{1}(\mathbb{T})$.

It is clear that this operator is well-defined, as $\{\hat{f}(n)\} \in c_{0}$ for every $f \in L^{1}(\mathbb{T})$ (RiemannLebesgue lemma ${ }^{1}$ ). And it is injective, which is equivalent to the Uniqueness theorem in Fourier series.

If we compute its norm, we obtain:

$$
\begin{aligned}
\|T\| & =\sup _{f \in \mathbb{S}_{L^{1}(\mathbb{T})}}\|T(f)\|=\sup _{f \in \mathbb{S}_{L^{1}(\mathbb{T})}\|\{\hat{f}(n)\}\|} \\
& =\sup _{f \in \mathbb{S}_{L^{1}(\mathbb{T})}} \sup _{n \in \mathbb{N}} \frac{1}{2 \pi}\left|\int_{-\pi}^{\pi} f(t) e^{-i n t} d t\right| \\
& \leq \sup _{f \in \mathbb{S}_{L^{1}(\mathbb{T})}} \sup _{n \in \mathbb{N}} \frac{1}{2 \pi} \int_{-\pi}^{\pi}|f(t)| d t \\
& =\sup _{f \in \mathbb{S}_{L^{1}(\mathbb{T})}}\|f\| \\
& =1
\end{aligned}
$$

${ }^{1}$ The Riemann-Lebesgue lemma states that if $f \in L^{1}(\mathbb{R})$, then the Fourier transform of $f$ satisfies

$$
\hat{f}(z)=\int_{\mathbb{R}^{d}} f(x) e^{-i z \cdot x} d x \rightarrow 0 \text { as }|z| \rightarrow \infty
$$

The version of the Riemann-Lebesgue lemma that we are using here is the following one: If $f$ is an integrable function on an interval, then the Fourier coefficients of $f$ tend to 0 as $n \rightarrow \pm \infty$.

Further information about this subject can be found in [DC01]. 
because the norm we are considering in $L^{1}(\mathbb{T})$ is $\|f\|=\frac{1}{2 \pi} \int_{-\pi}^{\pi}|f(t)| d t$.

Now, fixed $n_{0} \in \mathbb{N}$, if we consider $f(t)=e^{i n_{0} t}$, then,

$$
\|T(f)\|=\sup _{n \in \mathbb{N}}|\hat{f}(n)| \geq\left|\frac{1}{2 \pi} \int_{-\pi}^{\pi} f(t) e^{-i n_{0} t} d t\right|=\left|\frac{1}{2 \pi} \int_{-\pi}^{\pi} 1 d t\right|=1 .
$$

In conclusion, $\|T\|=1$ and $T$ attains its norm.

Incidentally, notice that $T$ cannot be surjective (as it is an isometry, if it were surjective, $T$ would be a linear homeomorphism between $L^{1}(\mathbb{T})$ and $c_{0}$, but their duals are $L^{\infty}(\mathbb{T})$ and $\ell_{1}$, respectively, and they cannot be homeomorphic, as the second one is separable and the first one not).

\subsubsection{EXTENSION OF THE BISHOP-PHELPS PROBLEM}

Notation 2.1. We will denote by $N A(X, Y)$ the subset of $L(X, Y)$ consisting of the operators which attain their norm. When $X$ and $Y$ are the same space, we denote $N A(X)=N A(X, X)$ and $L(X)=L(X, X)$.

In this section, we want to study the problem of determining for which Banach spaces $X$ and $Y$ the set $N A(X, Y)$ is dense in $L(X, Y)$. We will denote this property by

$$
\overline{N A(X, Y)}=L(X, Y) \quad(\overline{X Y}) .
$$

This problem is still unsolved in the general case, but there are several results for some particular situations. In the following sections, we will present some of these results and use them to get some examples of spaces satisfying $\overline{N A(X)}=L(X)$. This property will be denoted by

$$
\overline{N A(X)}=L(X) \quad(\bar{X})
$$

The first example of a space verifying property $(\bar{X})$ is $\mathbb{C}$ or $\mathbb{R}$. In the previous section we have seen that, for every Banach space $X$, the set of norm-attaining funtionals is dense. And this is verified, in particular, when $X=\mathbb{K}$. Then, we have:

Result 2.2.4 If $X=\mathbb{K}$, with $\mathbb{K}=\mathbb{C}$ or $\mathbb{R}$, then

$$
\overline{N A(X)}=L(X)
$$

The James Theorem states that a Banach space is reflexive if, and only if, every continuous linear functional attains its norm. In particular, as $\mathbb{K}$ is reflexive, we have

$$
N A(\mathbb{K})=L(\mathbb{K}) .
$$

Next, we want to show positive and negative answers for the property $(\overline{X Y})$, constructing examples from the properties we have already studied.

We saw in the previous section, as a consequence of the Bishop-Phelps theorem, that for every Banach space $X$, the set of norm-attaining funtionals in $X^{*}$ is dense for the norm topology. Rewriting that in the terms of this section, we obtain 


$$
\overline{N A(X, \mathbb{K})}=L(X, \mathbb{K})
$$

And when $X$ is reflexive, in virtue of James theorem, we have a better result,

$$
N A(X, \mathbb{K})=L(X, \mathbb{K})
$$

It is interesting to study not only the problem of the density, but also the problem of the equality of the sets $L(X, Y)$ and $N A(X, Y)$, for arbitrary Banach spaces $X$ and $Y$. We will denote this property by

$$
N A(X, Y)=L(X, Y) \quad(\sharp X Y) .
$$

And in the case that we are dealing with endomorphisms (domain and codomain are the same space), we will denote the previous property by $(\sharp X)$.

Before going ahead with the problem of the density, we will show some results about the problem of the equality.

For $Y$ a finite dimensional space, we can prove a result that can be considered an extension of James Theorem, Theorem 2.1.6. For its proof, we need the following lemma.

Lemma 2.2.5 Let $X$ and $Y$ be two Banach spaces. Let $T \in L(X, Y)$. Then, $T$ is continuous from $X$-weak ${ }^{a}$ to $Y$-weak. And the reciprocal is also true.

${ }^{a}$ We denote by $X$-weak the space $X$ with the weak topology.

Proof: $\Rightarrow)$ Let $f \in Y^{*}$, and consider the application $x \mapsto f(T x)$. It is linear and continuous from $X$ to $\mathbb{K}$, so it is continuous for the weak-topology of $X$.

As $f \circ T$ is continuous from $X$-weak to $\mathbb{K}$ for every $f \in Y^{*}, T$ is continuous from $X$-weak to $Y$-weak.

$\Leftarrow)$ Let $T$ be linear and continuous from $X$-weak to $Y$-weak. Then, the graph of $T, G(T)$, is closed in $X \times Y$ for the weak topology of the product space.

This implies that $G(T)$ is closed in $X \times Y$ for the strong topology. By the Closed Graph Theorem ${ }^{2}, T$ is continuous from $X$ to $Y$.

Now we can prove the following result.

Proposition 2.2.6 A Banach space $X$ is reflexive if, and only if, for every finite dimensional $Y$, every $T \in L(X, Y)$ attains its norm.

\footnotetext{
${ }^{2}$ For any function $T: X \rightarrow Y$, we define the graph of $T$ to be the set

$$
\{(x, y) \in X \times Y: T x=y\}
$$
}

The Closed Graph Theorem states: If $X$ and $Y$ are Banach spaces, and $T: X \rightarrow Y$ is a linear operator, then $T$ is continuous if, and only if, its graph is closed in $X \times Y$ with the product topology. 
Proof: $\Rightarrow)$ Let $Y$ be a finite dimensional space and consider $T \in L(X, Y)$. As $X$ is a reflexive space, $\mathbb{B}_{X}$ is weakly-compact, and as $T$ is continuous from $X$ to $Y$ with their respective topologies of the norm, for the previous lemma $T$ is also continuous from $X$-weak to $Y$-weak. Thus, $T$ takes weakly-compact sets in $X$ to weakly-compact sets in $Y$.

Therefore, $T\left(\mathbb{B}_{X}\right)$ is weakly-compact. Being $Y$ finite dimensional, the topology of the norm and the weak topology of $Y$ coincide. Then, $T\left(\mathbb{B}_{X}\right)$ is compact, so $T$ attains its norm.

$\Leftarrow)$ If every $T \in L(X, Y)$ attains its norm for every finite dimensional $Y$, in particular every functional in $X^{*}$ attains its norm. In virtue of James Theorem, $X$ is reflexive.

Therefore, every reflexive space $X$ and every finite dimensional $Y$ verify property $(\sharp X Y)$. $(\sharp X)$.

In particular, for $X$ a finite dimensional space, we have the following example for property

Result 2.2.7 Let $X$ be a finite dimensional Banach space. Then,

$$
N A(X)=L(X)
$$

In fact, for finite dimensional spaces we have a better result. Let $X$ be a finite dimensional space and $Y$ an arbitrary Banach space. Then, if $T \in L(X, Y)$, as $\mathbb{B}_{X}$ is compact for the topology of the norm and $T$ is a continuous operator, the set $T\left(\mathbb{B}_{X}\right)$ is compact in $Y$ for the topology of the norm, so $T$ attains its norm.

Then, we have just proved the following result.

Proposition 2.2.8 Let $X$ be a finite dimensional space and $Y$ an arbitrary Banach space. Then,

$$
N A(X, Y)=L(X, Y) \text {. }
$$

In virtue of Proposition 2.2.6, it is natural to ask whether 'finite dimensional' can be replaced by 'reflexive' in the above result. The answer is negative, since there is a bounded linear operator from $\ell_{2}(\mathbb{N})$ into itself that does not attain its norm.

- Example 2.2.9 Let $T$ be defined as

$$
\begin{aligned}
T: \ell_{2} & \longrightarrow \quad \ell_{2} \\
x & \mapsto T x:=\sum_{n \geq 1}\left(1-\frac{1}{n}\right)\left\langle x, e_{n}\right\rangle e_{n},
\end{aligned}
$$

where $\left\{e_{n}\right\}$ is the sequence whose $n$-th term is 1 and the others are 0 , and $\langle\cdot, \cdot\rangle$ is the scalar product of $\ell^{2}$. The norm of $T$ is 1 , but for $x \neq 0$,

$$
\|T x\|^{2}=\sum_{n \geq 1}\left(1-\frac{1}{n}\right)^{2}\left|\left\langle x, e_{n}\right\rangle\right|^{2}<\sum_{n \geq 1}\left|\left\langle x, e_{n}\right\rangle\right|^{2}=\|x\|^{2},
$$

since $\left\langle x, e_{n}\right\rangle \neq 0$ for some $n$.

We will prove in the next section that every reflexive Banach space has property $(\bar{X})$, as a consequence of the Lindenstrauss-Zizler theorem, Theorem 2.2.27. Therefore, we know that 
there are some reflexive spaces $X$ satisfying property $(\sharp X)$ (finite dimensional spaces), and some others that only verify the property $(\bar{X})$. Then, in general, we cannot extend what we proved for $X$ a finite dimensional space to $X$ a reflexive infinite dimensional space.

However, we can try to think now about the properties that $X$ and $Y$ must satisfy for verifying property $(\sharp X Y)$. Let us begin studying $X$. All the positive examples for this problem that we have already presented have in common that $X$ is a reflexive space. Therefore, the first question is if there exists a non-reflexive space $X$ such that for some $Y$, property $(\sharp X Y)$ holds.

The answer to this question is negative. Suppose that $X$ and $Y$ satisfy property $(\sharp X Y)$. Then, every $T \in L(X, Y)$ attains its norm. In particular, rank-one operators from $X$ into $Y$ attain their norm, so every functional in $X^{*}$ attains its norm. In virtue of James Theorem, $X$ is reflexive.

We have just proved the following result.

Proposition 2.2.10 Let $X$ and $Y$ be two Banach spaces verifying

$$
N A(X, Y)=L(X, Y)
$$

Then, $X$ is reflexive.

Therefore, we have a necessary condition for $X$ satisfying $(\sharp X Y)$ (remember that we saw in Proposition 2.2.8 that being finite dimensional is a sufficient condition for $X$ ). We have seen that, with $X$ being reflexive, a sufficient condition for $Y$ to satisfy property $(\sharp X Y)$ is to be finite dimensional. And for what we have seen in Example 2.2.9, being reflexive cannot be sufficient for $Y$.

Before introducing more sufficient conditions for $Y$, we can prove the following result, in order to know better the geometry of the set where we are studying whether the norm of the operator is attained or not.

Proposition 2.2.11 Let $X$ be a reflexive Banach space and $T \in L(X, Y)$, for $Y$ arbitrary. Then, $T\left(\mathbb{B}_{X}\right)$ is closed in $Y$ for the topology of the norm.

Proof: We have already proved in Proposition 2.2.6 that, as $X$ is reflexive, and this implies that $\mathbb{B}_{X}$ is weakly-compact, $T\left(\mathbb{B}_{X}\right)$ is weakly-compact.

The weak topology separates points, i.e., for every $y_{1}, y_{2} \in Y, y_{1} \neq y_{2}$, there exist $O_{1}, O_{2}$, open sets in the weak topology of $Y$, such that $y_{1} \in O_{1}, y_{2} \in O_{2}$ and $O_{1} \cap O_{2}=\emptyset$ (this is easy to see as a consequence of the Hahn-Banach Theorem). Then, $Y$ with the weak topology is a Hausdorff topological space. And as $T\left(\mathbb{B}_{X}\right)$ is weakly-compact, this implies that $T\left(\mathbb{B}_{X}\right)$ is weakly-closed.

However, every weakly-closed is also closed for the norm topology (the converse is true when the set we are studying is a convex). Then, $T\left(\mathbb{B}_{X}\right)$ is closed in $Y$ for the norm topology.

We have seen that the image of the ball of a reflexive space is closed, but this does not imply that the supremum in the definition of the norm of the operator is attained (as we have already presented in the example of the operator in $\ell^{2}$ ). In the finite dimensional case it is trivial to see that we have this implication, as every closed and bounded set is compact and the norm is a 
continuous application. Then, a good question to study is for which infinite dimensional spaces $Y$ the norm of an operator $T \in L(X, Y)$, with $X$ infinite dimensional and reflexive, is always attained.

We are going to present now a result, which appears in [JW79], that shows some sufficient and necessary conditions for $X$ and $Y$ to satisfy $(\sharp X Y)$. Before enunciating this result, we need some definitions.

\section{Definition 2.2.12 - COMPACT OPERATOR.}

Let $X$ and $Y$ be Banach spaces. We say that $T: X \rightarrow Y$ is compact if, and only if, one of the following equivalent statements is true

- $T\left(\mathbb{B}_{X}\right)$ is relatively compact in $Y$, i.e., $\overline{T\left(\mathbb{B}_{X}\right)}$ is compact in $Y$.

- $T(B)$ is relatively compact in $Y$ for every $B$ bounded set of $X$.

- For any sequence $\left\{x_{n}\right\} \subseteq \mathbb{B}_{X}$, the sequence $\left\{T x_{n}\right\}$ contains a Cauchy subsequence.

- There exists a neighbourhood of $0, U \subset X$, and a compact set $V \subset Y$ such that $T(U) \subset V$.

It is clear that if a linear operator is compact, then it is bounded. In fact, the set of compact operators constitutes a closed ideal of the set of bounded linear operators.

Notation 2.2. We denote the set of compact operators between two Banach spaces $X$ and $Y$ by $K(X, Y)$.

The set of compact operators will be essential in the study of norm-attaining operators. We will see more about this kind of operators in Chapter 5. For now, let us make the following remark. By Proposition 2.2.11, if $T$ is a bounded linear operator from a reflexive Banach space $X$ to an arbitrary Banach space $Y$, then $T\left(\mathbb{B}_{X}\right)$ is closed. Hence, if $T$ is compact, $T\left(\mathbb{B}_{X}\right)$ is compact, so $T$ attains its norm. Therefore, every compact operator from a reflexive Banach space $X$ to an arbitrary Banach space attains its norm.

Conversely, if $T$ is a bounded linear operator from an arbitrary $X$ to a finite dimensional space $Y, T\left(\mathbb{B}_{X}\right)$ is bounded, so $\overline{T\left(\mathbb{B}_{X}\right)}$ is compact (because $Y$ is finite-dimensional). Therefore, every bounded linear operator from an arbitrary Banach space $X$ to a finite dimensional space $Y$ is compact.

\section{Definition 2.2.13 - FINITE-RANK OPERATOR.}

Let $X$ and $Y$ be Banach spaces. We say that $T: X \rightarrow Y$ is a finite-rank operator if it is a bounded linear operator whose range is finite dimensional.

The set of finite-rank operators is also an ideal of the set of bounded linear ones.

Notation 2.3. We denote the set of finite-rank operators between two Banach spaces $X$ and $Y$ by $F(X, Y)$.

We will introduce now the approximation property. The idea of this property is that a space verifies it if every compact operator from it into an arbitrary Banach space is limite of finiterank operators between the same spaces. Although we will present this property in a different formulation, they are equivalent. Further information about this property can be found in Section 5.1 . 


\section{Definition 2.2.14 - APPROXIMATION PROPERTY.}

Let $X$ be a Banach space. We say that $X$ has the approximation property $(A P)$ if for every compact set $K \subset X$ and every $\varepsilon>0$ there exists an operator $T: X \rightarrow X$ of finite-rank such that

$$
\|T x-x\| \leq \varepsilon
$$

for every $x \in K$.

Now, we need to introduce the concept of atomic measure.

\section{Definition 2.2.15 - Atom, Atomic Measure.}

Let $\mu$ be a nonnegative measure on a $\sigma$-algebra $\mathscr{A}$ of subsets of a set $X$. An element $a \in \mathscr{A}$ is called an atom of $\mu$ if

- $\mu(A)>0$

- For every $B \in \mathscr{A}$ with $B \subset A$, either $\mu(B)=0$ or $\mu(B)=\mu(A)$

A $\sigma$-finite measure $\mu$ is called atomic if there is a partition of $X$ into countably many elements of $\mathscr{A}$ which are either atoms or null sets.

We also need the following result, proved by H. P. Rosenthal in [Ros69], which will be used to prove the result about spaces $X$ and $Y$ verifying $(\sharp X Y)$.

Theorem 2.2.16 Let $(X, \mathscr{A}, \mu)$ and $\left(X^{\prime}, \mathscr{A}^{\prime}, v\right)$ be measure spaces such that $L^{1}(\mu)$ and $L^{1}(v)$ are infinite dimensional. Let $1 \leq r, p<\infty$ with $r<p$.

Then, every bounded linear operator from a subspace of $L^{p}(\mu)$ to $L^{r}(v)$ is compact, in the following cases:

1. $\mu$ and $v$ are atomic.

2. $v$ is atomic and $1 \leq r<2$.

3. $\mu$ is atomic and $2<p$.

Moreover, if $1 \leq r, p<\infty$ and $p \leq r$ or if $r<p$ and $\mu$ and $v$ are such that $\mu, v, r$ and $p$ do not satisfy any of the conditions 1,2 or 3 , then there exists a noncompact bounded linear operator from $L^{p}(\mu)$ to $L^{r}(v)$.

Now we have the necessary tools to enunciate and demonstrate the following result.

Theorem 2.2.17 Let $X$ and $Y$ be two classical Banach spaces, i.e., they are of the form $L^{p}(\mu)$ or $C(S)$. Then, $N A(X, Y)=L(X, Y)$ if and only if $X=L^{p}(\mu), Y=L^{r}(v)$, with $1 \leq r<p<\infty$ and one of the following holds

(a) $1<r$ and $\mu$ and $v$ are atomic.

(b) $1<r<2$ and $v$ is atomic.

(c) $p>2, r>1$ and $\mu$ is atomic.

(d) $r=1$ and $v$ is atomic.

(e) $r=1, p>2$ and $\mu$ is atomic.

Proof: First, if $N A(X, Y)=L(X, Y)$, we have seen in Proposition 2.2.10 that $X$ is reflexive. Then, among the classical Banach spaces, $X$ can only be of the form $L^{p}(\mu)$, for $1<p<\infty$. 
Let us show now that $Y$ cannot be of the type $C(S)$. Suppose that we have $N A\left(X, c_{0}\right)=$ $L\left(X, c_{0}\right)$. Then, as $X$ is reflexive (and infinite dimensional, as finite dimensional spaces have been studied previously) there exists a sequence $\left\{x_{n}^{*}\right\} \subseteq \mathbb{S}_{X^{*}}$ such that $\left\{x_{n}^{*}\right\} \stackrel{\omega^{*}}{\longrightarrow} 0$ (this happens due to the Josefson-Nissenweig theorem, which appears in [Die84, Chapter 9]).

As appears in [MMP06], for example, if we take the operator $S: X \rightarrow c_{0}$ defined by

$$
[S x](n)=\frac{n}{n+1} x_{n}^{*}(x), \quad \forall x \in X, \forall n \in \mathbb{N} .
$$

Then, this operator has norm 1 and does not attain its norm, so we obtain a contradiction. And since $c_{0}$ embeds isometrically in any infinite dimensional $C(S)$ space, $Y$ cannot be of this type. Hence, $Y$ must also be of the type $L^{r}$.

Therefore, we are led to consider only $L^{p}$ spaces, i.e., $X=L^{p}(\mu)$ and $Y=L^{r}(v)$, with $1<p<\infty$ (because $X$ is reflexive).

Now, in [Hol73], it is proved that if $X$ and $Y$ are reflexive (now $1<r<\infty$ ) and one of them has the $A P$, the three following conditions are equivalent:

- $N A(X, Y)=L(X, Y)$.

- $L(X, Y)=K(X, Y)$.

- $L(X, Y)$ is reflexive.

Then, it is enough to study which $L^{p}$ spaces verify $L(X, Y)=K(X, Y)$, something that we have presented in Theorem 2.2.16.

The case $r>1$ is an immediate consequence of conditions 1, 2 and 3 in this theorem, so this yields the results of the cases (a), (b) and (c), respectively.

For $r=1$, it is easy to see that every compact operator from a reflexive space to $L^{1}(v)$, with $v$ an atomic measure, attains its norm. Then, again the second condition of Theorem 2.2.16 yields the sufficiency of (d), and the third condition the sufficiency of (e).

It remains to show that if $r=1$, (d) and (e) are necessary. We will see it in the following two examples, due to J. Bourgain.

- Example 2.2.18 For $1<p<2$, we define the operator $T: \ell_{p} \rightarrow L^{1}[0,1]$ by

$$
T x=\sum_{n=1}^{\infty}\left(1-\frac{1}{n}\right) x_{n} p_{n}
$$

where $x=\left\{x_{n}\right\}$ and $\left\{p_{n}\right\}$ is the sequence of Rademacher functions ${ }^{3}$ on $[0,1]$. Then,

$$
\|T x\|_{1} \leq\|T x\|_{2}=\left[\sum_{n=1}^{\infty}\left(1-\frac{1}{n}\right)^{2} x_{n}^{2}\right]^{1 / 2}<\left[\sum_{n=1}^{\infty} x_{n}^{2}\right]^{1 / 2} \leq\|x\|_{p},
$$

where the last inequality comes from the fact that $p<2$. Thus, it is clear that $\|T\| \leq 1$.

\footnotetext{
${ }^{3}$ The Rademacher functions constitute the Rademacher system, which is an incomplete orthogonal system of functions of the unit interval of the following form:

$$
\left\{t \mapsto r_{n}(t)=\operatorname{sgn}\left(\operatorname{sen}\left[2^{n+1} \pi t\right]\right): t \in[0,1], n \in \mathbb{N}\right\}
$$
}


If we take $\left\{e_{n}\right\}$, the elements of the canonical basis of $\ell_{p}$, then $T e_{n}=(1-1 / n) p_{n}$ for every $n \in \mathbb{N}$, so $\|T\|=1$. However, the above inequality shows that the norm is not attained.

- Example 2.2.19 For $2 \leq p<\infty$, we define the operator $T: L^{p}[0,1] \rightarrow L^{1}[0,1]$ by

$$
T f=\sum_{n=1}^{\infty}\left(1-\frac{1}{n}\right)\left[\int_{0}^{1} f p_{n} d x\right] p_{n}
$$

where $\left\{p_{n}\right\}$ are again the Rademacher functions. Then, if we consider the inclusions

$$
I_{1}: L^{p} \rightarrow L^{2} \quad \text { and } \quad I_{2}: L^{2} \rightarrow L^{1}
$$

and also $P$ the projection of $L^{2}$ on the span of $\left\{p_{n}\right\}$ and $S$ an application that maps the span of the Rademacher functions onto itself by $S\left(\sum a_{n} p_{n}\right)=\sum(1-1 / n) a_{n} p_{n}$, we have $T=I_{2} S P I_{1}$.

Since each of these operators has norm 1 , so does $T$. However, $T$ does not attain its norm, since $S$ does not.

It is easy to show that if $H$ is the range of a norm one projection on $E$, then the condition $N A(E, F)=L(E, F)$ implies $N A(H, F)=L(H, F)$. Hence, for the first of the examples we have just seen, $N A\left(L^{p}, L^{1}\right) \neq L\left(L^{p}, L^{1}\right)$ for $1<p<2$. This completes the proof of the previous theorem.

As a consequence of this theorem, we obtain the following result. We have proved that, among the classical Banach spaces of the type $C(S)$ and $L^{p}(\mu)$, the only ones satisfying $N A(X, Y)=$ $L(X, Y)$ are spaces of the type $X=L^{p}(\mu)$ and $Y=L^{r}(v)$, where $r<p$. Then, for every space $X$ of the type $C(S)$ or $L^{p}(\mu)$, we have

$$
N A(X) \neq L(X)
$$

In particular, this class of spaces contains all Hilbert spaces, which we have already seen in 2.2.9 that could not satisfy this property.

Therefore, the only spaces verifying $N A(X)=L(X)$ we have found are the finite dimensional ones.

Now we go back to the problem of density of the set of norm-attaining operators between two Banach spaces $X$ and $Y$ in the set of bounded linear operators between the same two spaces.

It is clear that all the examples of spaces $X$ and $Y$ verifying $N A(X, Y)=L(X, Y)$ are, in particular, examples of $\overline{N A(X, Y)}=L(X, Y)$. Then, let us expose now the examples we already know of spaces satisfying this property.

At the beginning of the chapter we mentioned the Bishop-Phelps theorem, which states that

$$
\overline{N A(X, \mathbb{K})}=L(X, \mathbb{K})
$$

for every Banach space $X$. Later, we proved that if a space $X$ is reflexive, and $Y$ is finite dimensional, then $N A(X, Y)=L(X, Y)$; in particular,

$$
\overline{N A(X, Y)}=L(X, Y) \text {. }
$$


An interesting fact to remark is that, even though we could try to think of an extension of Bishop-Phelps theorem when the codomain is a finite dimensional space with dimension greater than one, as we did with James theorem, it remains an open problem to know if this could be done.

In fact, we do not know yet if there exists a Banach space $X$ such that $\overline{N A\left(X,\left(\mathbb{R}^{2},\|\cdot\|_{2}\right)\right)} \neq$ $L\left(X,\left(\mathbb{R}^{2},\|\cdot\|_{2}\right)\right.$ ) (or, on the contrary, $\overline{N A\left(X,\left(\mathbb{R}^{2},\|\cdot\|_{2}\right)\right)}=L\left(X,\left(\mathbb{R}^{2},\|\cdot\|_{2}\right)\right.$ ) for every Banach space $X$ ), where in $\mathbb{R}^{2}$ we are considering the euclidean norm. If we consider in $\mathbb{R}^{2}$ other norms, the previous result may be true (for instance, considering $\|\cdot\|_{1}$ or $\|\cdot\|_{\infty}$ ), as we will see in the following chapter that a finite dimensional space has property $\beta$ if, and only if, its unit ball is polyhedral, so if we consider in $\mathbb{R}^{2}$ a norm $\|\cdot\|$ such that the unit ball of $\left(\mathbb{R}^{2},\|\cdot\|\right)$ is a polyhedron, then, in particular, $\overline{N A\left(X,\left(\mathbb{R}^{2},\|\cdot\|\right)\right)}=L\left(X,\left(\mathbb{R}^{2},\|\cdot\|\right)\right)$ for every Banach space $X$. Further, we do not know whether a result so natural as 'for every Banach space $X$, there exists a norm-attaining operator from $X$ to $\left(\mathbb{R}^{2},\|\cdot\|_{2}\right)^{\prime}$ is true or false.

Finally, in Theorem 2.2.17 we have seen five cases of spaces $L^{p}(\mu)$ and $L^{r}(v)$ verifying $N A\left(L^{p}(\mu), L^{r}(v)\right)=L\left(L^{p}(\mu), L^{r}(v)\right)$. Therefore, for $X=L^{p}(\mu)$ and $Y=L^{r}(v)$, if one of the following holds:

- $1<r$ and $\mu$ and $v$ are atomic.

- $1<r<2$ and $v$ is atomic.

- $p>2, r>1$ and $\mu$ is atomic.

- $r=1$ and $v$ is atomic.

- $r=1, p>2$ and $\mu$ is atomic.

then we have $\overline{N A(X, Y)}=L(X, Y)$.

In the following sections and chapters we will develop some tools to find more positive and negative examples for this problem. We will begin by showing, in the next section, the first negative example found for this problem.

\subsubsection{FiRSt NEGATIVE EXAMPLE}

In 1963, Lindenstrauss opened a new field with the research on the problem $\overline{N A(X, Y)}=L(X, Y)$, a field to which many other authors would also contribute with their results. Lindenstrauss presented some counterexamples to show that $N A(X, Y)$ might not be dense in $L(X, Y)$. The underlying idea of the construction is the following one:

Let us suppose that $Y$ is strictly convex and let $T \in L(X, Y)$ be an injective operator which attains its norm in $x_{0} \in \mathbb{S}_{X}$. Then, $x_{0}$ has to be an extreme point of $\mathbb{B}_{X}$, because if $x \in X$ and $\left\|x_{0} \pm x\right\| \leq 1$, we have

$$
\left\|T x_{0} \pm T x\right\| \leq\|T\|=\left\|T x_{0}\right\|,
$$

and, as $Y$ is strictly convex, $T x=0$, so $x=0$. For that, if $\mathbb{B}_{X}$ lacks extreme points, no injective operator from $X$ to $Y$ attains its norm. If $X$ and $Y$ are isomorphic, the set of isomorphisms between $X$ and $Y$ is open in $L(X, Y)$, and, by the previous argument, it has empty intersection with $N A(X, Y)$, so $N A(X, Y)$ is not dense.

Renorming some Banach spaces with an equivalent strictly convex norm is not a difficult job. To this end, we can use, for instance, the following lemma. 
Lemma 2.2.20 Let $X$ and $Y$ be two Banach spaces, $Y$ strictly convex, and let $T \in L(X, Y)$ be an injective operator. Then, the norm defined in $X$ by

$$
|x|=\|x\|+\|T x\| \quad(x \in X)
$$

is strictly convex.

Proof: Let $x_{1}, x_{2} \in X$ such that

$$
\left|x_{1}\right|=\left|x_{2}\right|=1, \quad\left|x_{1}+x_{2}\right|=2 .
$$

Rewriting this is terms of $\|\cdot\|$, we have,

$$
\left\|x_{1}\right\|+\left\|T x_{1}\right\|=\left\|x_{2}\right\|+\left\|T x_{2}\right\|=1, \quad\left\|x_{1}+x_{2}\right\|+\left\|T x_{1}+T x_{2}\right\|=2 .
$$

Using the triangular inequiality in this expression, it is clear that

$$
\left\|T x_{1}+T x_{2}\right\|=\left\|T x_{1}\right\|+\left\|T x_{2}\right\| .
$$

As $Y$ is strictly convex, there exists a constant $\lambda \geq 0$ such that

$$
T x_{1}=\lambda T x_{2}
$$

And as $T$ is injective, $x_{1}=\lambda x_{2}$. The definition of $x_{1}$ and $x_{2}$ shows us that $\lambda=1$ and, hence, $x_{1}=x_{2}$.

The condition of Lemma 2.2.20 seems not so hard, but it is not satisfied for every Banach space. In fact, there are Banach spaces admitting no equivalent strictly convex norm. One example for this is the space $\ell_{\infty}$.

For a particular example of $\overline{N A(X, Y)} \neq L(X, Y)$, let us just consider a Banach space $X$ whose unit ball has no extreme point and can be renormed with a strictly convex norm. The space $c_{0}$ has this property. Following the previous lemma, we consider $c_{0}$ with the norm $|\cdot|$ given by:

$$
|x|=\|x\|_{\infty}+\left(\sum_{n=1}^{\infty} \frac{|x(n)|^{2}}{2^{n}}\right)^{\frac{1}{2}} \quad\left(x \in c_{0}\right),
$$

where

$$
\begin{aligned}
T: c_{0} & \rightarrow l_{2} \\
x & \mapsto\left\{\frac{x(n)}{2^{n / 2}}\right\} \quad\left(x \in c_{0}, n \in N\right)
\end{aligned}
$$

is an injective operator, $\|\cdot\|_{\infty}$ is the usual norm in $c_{0}$ and $x(n)$ the $\mathrm{n}$-th term of the sequence $x \in c_{0}$, $\forall n \in \mathbb{N}$. Then, we obtain a strictly convex space. This can be seen in [Cla36].

As $\left(c_{0},\|\cdot\|_{\infty}\right)$ has no extreme points and $\left(c_{0},|\cdot|\right)$ is strictly convex, for what we have seen above it is clear that $\overline{N A\left(\left(c_{0},\|\cdot\|_{\infty}\right),\left(c_{0},|\cdot|\right)\right)} \neq L\left(\left(c_{0},\|\cdot\|_{\infty}\right),\left(c_{0},|\cdot|\right)\right)$. In fact, for $c_{0}$ we can improve this result with the following proposition: 
Proposition 2.2.21 Let $Y$ be a strictly convex Banach space. If $T \in N A\left(c_{0}, Y\right)\left(c_{0}\right.$ with its usual norm), then

$$
\exists p \in \mathbb{N}: n \geq p \Rightarrow T e_{n}=0,
$$

where $\left\{e_{n}\right\}$ is the canonical basis of $c_{0}$. In particular, $T$ is a finite rank operator.

Proof: As $T \in N A\left(c_{0}, Y\right)$, there exists $x_{0} \in \mathbb{S}_{c_{0}}$ such that $\left\|T x_{0}\right\|=\|T\|$. We choose $p \in \mathbb{N}$ so that

$$
\left|x_{0}(n)\right|<\frac{1}{2} \quad \text { for } n \geq p .
$$

Now, as $\left\|x_{0} \pm \frac{1}{2} e_{n}\right\| \leq 1$, we obtain that $\left\|T x_{0} \pm \frac{1}{2} T e_{n}\right\| \leq\|T\|=\left\|T x_{0}\right\|$, and by the strict convexity of $Y, T e_{n}=0$ for $n \geq p \Rightarrow T$ is a finite rank operator.

We have proved that $N A\left(c_{0}, Y\right) \subseteq F\left(c_{0}, Y\right)$ and we know that $F\left(c_{0}, Y\right) \subseteq K\left(c_{0}, Y\right)$. As $K\left(c_{0}, Y\right)$ is closed in $L\left(c_{0}, Y\right)$, if we take closures in $L\left(c_{0}, Y\right)$, we have

$$
\overline{N A\left(c_{0}, Y\right)} \subseteq \overline{F\left(c_{0}, Y\right)} \subseteq K\left(c_{0}, Y\right) .
$$

As a consequence of this proposition, if there exists a non compact operator from $c_{0}$ to $Y$, then $T \notin \overline{N A\left(c_{0}, Y\right)}$, so $N A\left(c_{0}, Y\right)$ is not dense in $L\left(c_{0}, Y\right)$.

Finally, using the same idea of the previous proof, we can find our first example of a Banach space $X$ such that $N A(X)$ is not dense in $L(X)$. It is shown in the following proposition.

Proposition 2.2.22 Let $(Y,|\cdot|)$ be a strictly convex Banach space isomorphic to $c_{0}$, and let $X=Y \oplus_{\infty} c_{0}$, where $c_{0}$ has the usual norm, and the norm of the direct sum is the norm of the maximum:

$$
\|y+z\|=\max \{|y|,\|z\|\} \quad\left(y \in Y, z \in c_{0}\right) .
$$

Then, $N A(X)$ is not dense in $L(X)$.

The proof of this proposition appears in [Lin63]. We are going to explain it now.

Proof: Let $T_{0}$ be an isomorphism from $c_{0}$ onto $Y$ with $\left\|T_{0}\right\| \leq 1$. For some $\varepsilon>0$, we have $\left|T_{0} z\right| \geq 2 \varepsilon\|z\| \forall z \in c_{0}$. Define $T: X \rightarrow X$ by $T(y, z)=\left(T_{0} z, 0\right)$.

Let us suppose that $N A(X)$ is dense in $L(X)$. Then, there exists $\hat{T}: X \rightarrow X$ bounded and linear such that $\|\hat{T}-T\|<\varepsilon$ and $\hat{T}$ attains its norm in $\left(y_{0}, z_{0}\right) \in \mathbb{S}_{X}$. We call $\hat{T}\left(y_{0}, z_{0}\right)=(u, v)$. By the definition of $T$ and $\hat{T}$, clearly $\|v\|<\varepsilon$; and since $\|\hat{T}\|>\varepsilon$, it follows that $\|v\| \leq\|\hat{T}\|=|u|$.

As $\mathbb{S}_{c_{0}}$ has no extreme points, there exists $z_{1} \neq 0$ such that

$$
\left\|z_{1}+z_{0}\right\|=\left\|-z_{1}+z_{0}\right\| \leq 1
$$

Hence,

$$
\left\|\hat{T}\left(y_{0}, z_{0}\right) \pm \hat{T}\left(0, z_{1}\right)\right\|=\left\|\hat{T}\left(y_{0}, z_{0} \pm z_{1}\right)\right\| \leq\|\hat{T}\| .
$$

Since $Y$ is strictly convex and $|u|=\|\hat{T}\|$, it follows that $\hat{T}\left(0, z_{1}\right)=\left(0, z_{2}\right)$ for some $z_{2} \in c_{0}$ (because $\hat{T}$ attains its norm at $\left.\left(y_{0}, z_{0}\right)\right)$. Then, as $\|\hat{T}-T\|<\varepsilon$, we get 


$$
\varepsilon\left\|z_{1}\right\| \geq\left\|\hat{T}\left(0, z_{1}\right)-T\left(0, z_{1}\right)\right\| \geq\left|T_{0} z_{1}\right| \geq 2 \varepsilon\left\|z_{1}\right\|
$$

And, as $\varepsilon>0$, this is a contradiction.

Now, we have already proved the first counterexample for the situation $\overline{N A(X)}=L(X)$.

Result 2.2.23 If $(Y,|\cdot|)$ is a strictly convex Banach space isomorphic to $c_{0}$ and $X=Y \oplus_{\infty} c_{0}$, then

$$
\overline{N A(X)} \neq L(X)
$$

\subsubsection{THE LiNDENSTRAUSS-ZiZLER THEOREM}

In this section, we continue the study of the problem $\overline{N A(X, Y)}=L(X, Y)$ for certain Banach spaces $X$ and $Y$. We have just seen that this expression is not true in general. Now, we want to find properties for $X$ or $Y$ that make the previous relation true.

As we are dealing with an optimization problem, we can expect better conditions in the dual spaces, as we have there arguments for compactness in the weak-* topology.

Let $X$ and $Y$ be two Banach spaces and $T \in L(X, Y)$ an operator. Then, we can consider the adjoint operator $T^{*} \in L\left(Y^{*}, X^{*}\right)^{4}$. It is easy to see that if $T$ attains its norm, the same happens to $T^{*}$ (but the reciprocal is not true).

Now, we can consider the following sets:

$$
\begin{gathered}
N A_{1}(X, Y)=\left\{T \in L(X, Y): T^{*} \in N A\left(Y^{*}, X^{*}\right)\right\}, \\
N A_{2}(X, Y)=\left\{T \in L(X, Y): T^{* *} \in N A\left(X^{* *}, Y^{* *}\right)\right\} .
\end{gathered}
$$

Then, we have

$$
N A(X, Y) \subseteq N A_{1}(X, Y) \subseteq N A_{2}(X, Y)
$$

And we can easily see that these inclusions may be strict. For that, let us consider the operator

$$
T: c_{0} \rightarrow c_{0}
$$

given by

$$
T x=\sum_{n=1}^{\infty}\left(1-\frac{1}{n}\right) x(n) e_{n} \quad\left(x \in c_{0}\right)
$$

where $\left\{e_{n}\right\}$ is the canonical basis of $c_{0}$. We will see that $T^{* *}$ attains its norm, but $T^{*}$ does not. Using the canonical identification $c_{0}^{*} \equiv \ell_{1}$, the adjoint operator can be expressed as:

\footnotetext{
${ }^{4}$ Let $X$ and $Y$ be Banach spaces and $T \in L(X, Y)$. Then, we define the adjoint operator of $T$, and denote it by $T^{*}$, as the bounded linear operator $T^{*}: Y^{*} \rightarrow X^{*}$ such that

$$
y^{*}(T x)=\left(T^{*} y^{*}\right)(x), \quad \forall x \in X, \forall y^{*} \in Y^{*}
$$
}




$$
T^{*} x^{*}=\sum_{n=1}^{\infty}\left(1-\frac{1}{n}\right) x^{*}(n) f_{n} \quad\left(x^{*} \in \ell_{1}\right),
$$

where $\left\{f_{n}\right\}$ is the canonical basis of $\ell_{1}$ seen as the dual of $c_{0}$, i.e., $f_{n}\left(e_{m}\right)=\delta_{n, m}$ (it is easy to check, in the previous expressions, that $x^{*}(T x)=\left(T^{*} x^{*}\right) x$ for every $x \in c_{0}$ and every $\left.x^{*} \in \ell_{1}\right)$. Then, it is clear that $T^{*}$ does not attain its norm, as evaluating $T^{*}$ in $\left\{f_{n}\right\}$, we can see that $\left\|T^{*}\right\|=1$, but for every $x^{*} \in \mathbb{S}_{\ell_{1}}$,

$$
\left\|T^{*} x^{*}\right\|_{1}=\sum_{n=1}^{\infty}\left|1-\frac{1}{n}\right|\left|x^{*}(n)\right|<\sum_{n=1}^{\infty}\left|x^{*}(n)\right|=\left\|x^{*}\right\|_{1},
$$

and this inequality is strict, as at least one term is different from zero.

However, if we consider $T^{* *}$, as $\ell_{1}^{*} \equiv \ell_{\infty}$, then $T^{* *} \in L\left(\ell_{\infty}\right)$ takes the form

$$
T^{* *} x^{* *}=\sum_{n=1}^{\infty}\left(1-\frac{1}{n}\right) x^{* *}(n) g_{n} \quad\left(x^{* *} \in \ell_{\infty}\right),
$$

where $\left\{g_{n}\right\}$ verifies $g_{n}\left(f_{m}\right)=\delta_{n, m}$ for all $n, m \in \mathbb{N}$. Taking $x^{* *} \in \ell_{\infty}$ such that $x^{* *}(n)=1 \forall n \in \mathbb{N}$, it is clear that $T$ attains its norm.

Lindenstrauss also showed in [Lin63], that for every Banach spaces $X$ and $Y$, the set $N A_{2}(X, Y)$ is dense in $L(X, Y)$. Ten years later, Zizler [Ziz73] improved this result, showing that, in fact, $N A_{1}(X, Y)$ is always dense in $L(X, Y)$.

We will show below the proof of Zizler's result, which is nothing but an ingenious modification of Lindenstrauss' proof. We need a preparatory lemma, [Ziz73, Lemma 1], whose proof is a direct consequence of Banach-Alaoglú Theorem (the analogous lemma, necessary for the proof of Lindenstrauss' result, is a consequence of Goldstine Theorem).

Theorem 2.2.24 - BANACH-ALAOGLÚ THEOREM.

Let $X$ be a Banach space. Then, $\mathbb{B}_{X^{*}}$ is compact in the $\omega^{*}$ topology.

Theorem 2.2.25 - GOLDSTINE THEOREM.

Let $X$ be a Banach space. Consider $J: X \rightarrow X^{* *}$ the canonical embedding of $X$ in its bidual. Then, the image of $\mathbb{B}_{X}$ under this embedding into $\mathbb{B}_{X^{* *}}$ is $\omega^{*}$-dense.

Now we can enunciate and prove the lemma mentioned above.

Lemma 2.2.26 Let $X$ and $Y$ be Banach spaces and $T \in L(X, Y)$. Then, $T \in N A_{1}(X, Y)$ if and only if there exist $\left\{x_{n}\right\}_{n \in \mathbb{N}} \subset \mathbb{S}_{X}$ and $\left\{y_{n}^{*}\right\}_{n \in \mathbb{N}} \subset \mathbb{S}_{Y^{*}}$ such that

$$
\left|y_{n}^{*}\left(T x_{m}\right)\right| \geq\|T\|-\frac{1}{m} \quad \forall n, m \in \mathbb{N} \quad \text { with } m<n .
$$

Proof: $\Rightarrow)$ Let $T \in N A_{1}(X, Y)$. Then, $Y^{*}$ attains its norm at $y^{*} \in \mathbb{S}_{Y^{*}}$, so if we take $y_{n}^{*}=y^{*}$ for every $n \in \mathbb{N}$, and as $\left\|T^{*} y^{*}\right\|=\|T\|$, we can choose for every $m, x_{m} \in \mathbb{S}_{X}$ such that

$$
\left|\left(T^{*} y^{*}\right)\left(x_{m}\right)\right| \geq\|T\|-\frac{1}{m} .
$$


$\Leftarrow$ ) Considering the sequence $\left\{y_{n}^{*}\right\}_{n \in \mathbb{N}} \subset \mathbb{S}_{Y^{*}}$, as $\mathbb{B}_{Y^{*}}$ is $\omega^{*}$-compact (Banach-Alaoglú Theorem), there exists an adherent point for the sequence, $y^{*} \in \mathbb{B}_{Y^{*}}$. Then, as $\left\{x_{n}\right\}_{n \in \mathbb{N}} \subset \mathbb{S}_{X}$ and $\left\{y_{n}^{*}\right\}_{n \in \mathbb{N}} \subset \mathbb{S}_{Y^{*}}$ verify

$$
\left|y_{n}^{*}\left(T x_{m}\right)\right| \geq\|T\|-\frac{1}{m} \quad \forall n, m \in \mathbb{N} \text { with } m<n,
$$

we have

$$
\left\|T^{*} y^{*}\right\| \geq\left|y^{*}\left(T x_{m}\right)\right| \geq\|T\|-\frac{1}{m} \quad \forall m \in \mathbb{N} .
$$

Hence,

$$
\left\|T^{*} y^{*}\right\|=\|T\|
$$

Finally, we can enunciate and prove the result of Zizler.

Theorem 2.2.27 — LINDENStRAuSS-ZIZLER THeOREM.

Let $X$ and $Y$ be Banach spaces. Then, the set $N A_{1}(X, Y)$ of the operators from $X$ to $Y$ whose adjoints attain their norm is dense in $L(X, Y)$.

Proof: Let $T \in L(X, Y)$ with $\|T\|=1$ and $\varepsilon \in(0,1 / 3)$. We will construct an operator $S$ whose adjoint attains its norm verifying

$$
\|T-S\|<\varepsilon .
$$

First, we choose a decreasing sequence $\left\{\varepsilon_{k}\right\}, k=1,2, \ldots$ of positive numbers such that

$$
\sum_{i=1}^{\infty} \varepsilon_{i}<\frac{\varepsilon}{2}, \quad 2 \sum_{i=n+1}^{\infty} \varepsilon_{i}<\frac{\varepsilon_{n}^{2}}{2}, \quad \varepsilon_{n}<\frac{1}{10(n+1)}, \quad \forall n \in \mathbb{N} .
$$

Next, we define inductively a sequence $\left\{T_{n}\right\}_{n \in \mathbb{N}}$ of linear bounded operators from $X^{*}$ into $Y^{*}$ and sequences $\left\{x_{n}\right\}_{n \in \mathbb{N}} \subset \mathbb{S}_{X}$ and $\left\{y_{n}^{*}\right\}_{n \in \mathbb{N}} \subset \mathbb{S}_{Y^{*}}$ such that

$$
T_{1}=T
$$

and, for every step $n \in \mathbb{N}$,

$$
\left\|T_{n}^{*} y_{n}^{*}\right\| \geq\left\|T_{n}\right\|-\varepsilon_{n}^{2}
$$

and

$$
y_{n}^{*}\left(T_{n} x_{n}\right)=\left|y_{n}^{*}\left(T_{n} x_{n}\right)\right| \geq\left\|T_{n}^{*} y_{n}^{*}\right\|-\varepsilon_{n}^{2} .
$$

Now, defining

$$
T_{n+1} x=T_{n} x+\varepsilon_{n} y_{n}^{*}\left(T_{n} x\right) T_{n} x_{n},
$$

since $\|T\| \leq\left\|T_{n}\right\|+\varepsilon_{n}\left\|T_{n}\right\|^{2}$ and 


$$
\left(1+\sum_{i=1}^{n-1} 2 \varepsilon_{i}\right)^{2} \leq(1+\varepsilon)^{2} \leq\left(\frac{4}{3}\right)^{2}<2,
$$

it is easy to prove inductively that for every $n \in \mathbb{N}$

$$
\left\|T_{n+1}\right\| \leq 1+\sum_{i=1}^{n} 2 \varepsilon_{i} \leq \frac{4}{3}
$$

Taking into account that

$$
\left\|T_{n+1}-T_{m}\right\|=\operatorname{Sup}_{x \in \mathbb{S}_{X}}\left\|T_{n} x+\varepsilon_{n} y_{n}^{*}\left(T_{n} x\right) T_{n} x_{n}-T_{m} x\right\| \leq\left\|T_{n}-T_{m}\right\|+\varepsilon\left\|T_{n}\right\|^{2},
$$

we can also prove by induction that

$$
\left\|T_{n+1}-T_{m}\right\| \leq \sum_{i=m}^{n} 2 \varepsilon_{i} \forall m \leq n .
$$

Hence, the sequence $\left\{T_{n}\right\}_{n \in \mathbb{N}}$ converges in the norm topology to $S \in L(X, Y)$, satisfying

$$
\left\|T_{m}-S\right\| \leq 2 \sum_{i=m}^{\infty} \varepsilon_{i}<\varepsilon_{m-1} \quad \forall m \in \mathbb{N} .
$$

In particular, $\|S-T\|<\varepsilon$. Now, we will prove that $S^{*}$ attains its norm. From the definition of $T_{n+1}$ in terms of $T_{n}$, we can see that

$$
T_{n+1}^{*} y^{*}=T_{n}^{*} y^{*}+\varepsilon_{n}\left[\left(T_{n}^{*} y^{*}\right)\left(x_{n}\right)\right] T_{n}^{*} y_{n}^{*} \quad\left(y^{*} \in Y^{*}\right) .
$$

Then, using the conditions in the definition of $\left\{T_{n}\right\}_{n \in \mathbb{N}},\left\{x_{n}\right\}_{n \in \mathbb{N}},\left\{y_{n}^{*}\right\}_{n \in \mathbb{N}}$, we can prove:

$$
\begin{array}{|cr|}
\left\|T_{n+1}\right\| \geq\left\|T_{n}\right\|+\varepsilon_{n}\left\|T_{n}\right\|^{2}-5 \varepsilon_{n}^{2} & \forall n \in \mathbb{N} \\
\left\|T_{m+1}^{*} y_{n}^{*}\right\| \geq\left\|T_{m+1}\right\|-2 \varepsilon_{m}^{2} & \forall m<n
\end{array} .
$$

For the first one:

$$
\begin{aligned}
\left\|T_{n+1}\right\| \geq\left\|T_{n+1}^{*} y_{n}^{*}\right\| & =\left\|T_{n}^{*} y_{n}^{*}\right\|\left(1+\varepsilon_{n}\left(T_{n}^{*} y_{n}^{*}\right)\left(x_{n}\right)\right) \\
& \geq\left(\left\|T_{n}\right\|-\varepsilon_{n}^{2}\right)\left(1+\varepsilon_{n}\left\|T_{n}\right\|-2 \varepsilon_{n}^{3}\right) \\
& \geq\left\|T_{n}\right\|+\varepsilon_{n}\left\|T_{n}\right\|^{2}-\varepsilon_{n}^{2}\left(1+3 \varepsilon_{n}\left\|T_{n}\right\|\right) \\
& \geq\left\|T_{n}\right\|+\varepsilon_{n}\left\|T_{n}\right\|^{2}-5 \varepsilon_{n}^{2} .
\end{aligned}
$$

And as a consequence, the sequence $\left\{\left\|T_{n}\right\|\right\}_{n \in \mathbb{N}}$ is increasing. For the second one, if $m<n$ :

$$
\begin{aligned}
\left\|T_{m+1}^{*} y_{n}^{*}\right\| & \geq\left\|T_{n}^{*} y_{n}^{*}\right\|-\left\|T_{n}-T_{m+1}\right\| \\
& \geq\left\|T_{n}\right\|-\varepsilon_{n}^{2}-\varepsilon_{m}^{2} \\
& \geq\left\|T_{n}\right\|-2 \varepsilon_{m}^{2} \\
& \geq\left\|T_{m+1}\right\|-2 \varepsilon_{m}^{2} .
\end{aligned}
$$

Now, as $\left\|T_{m+1}^{*} y_{n}^{*}\right\| \leq\left\|T_{m}\right\|+\varepsilon_{m}\left|\left(T_{m}^{*} y_{n}^{*}\left(x_{m}\right)\right)\right|\left\|T_{m}\right\|$, if $m<n$, putting together the two expression we have just proved, 


$$
\begin{aligned}
\left\|T_{m}\right\|+\varepsilon_{m}\left|\left(T_{m}^{*} y_{n}^{*}\left(x_{m}\right)\right)\right|\left\|T_{m}\right\| & \geq\left\|T_{m+1}^{*} y_{n}^{*}\right\| \\
& \geq\left\|T_{m+1}\right\|-2 \varepsilon_{m}^{2} \\
& \geq\left\|T_{m}\right\|+\varepsilon_{m}\left\|T_{m}\right\|^{2}-7 \varepsilon_{m}^{2} .
\end{aligned}
$$

Then, for every $m<n$,

$$
\varepsilon_{m}\left|\left(T_{m}^{*} y_{n}^{*}\left(x_{m}\right)\right)\right|\left\|T_{m}\right\| \geq \varepsilon_{m}\left\|T_{m}\right\|^{2}-7 \varepsilon_{m}^{2} .
$$

And dividing by $\varepsilon_{m}\left\|T_{m}\right\|$, we have,

$$
\left|y_{n}^{*}\left(T_{m} x_{m}\right)\right| \geq\left\|T_{m}\right\|-\frac{7 \varepsilon_{m}^{2}}{\varepsilon_{m}\left\|T_{m}\right\|} \stackrel{\left\|T_{m}\right\| \geq 1}{\geq}\left\|T_{m}\right\|-7 \varepsilon_{m} .
$$

We want to prove that $S$ verifies the hypothesis of the previous lemma. Using this inequality and some previous estimations,

$$
\begin{aligned}
\left|y_{n}^{*}\left(S x_{m}\right)\right| & \geq\left|y_{n}^{*}\left(T_{m} x_{m}\right)\right|-\left|y_{n}^{*}\left(T_{m} x_{m}\right)-y_{n}^{*}\left(S x_{m}\right)\right| \\
& \geq\left\|T_{m}\right\|-7 \varepsilon_{m}-\left\|T_{m}-S\right\| \\
& \geq\left\|T_{m}\right\|-7 \varepsilon_{m}-\varepsilon_{m-1}^{2} \\
& \geq\|S\|-8 \varepsilon_{m}-2 \varepsilon_{m-1}^{2} \\
& \geq\|S\|-10 \varepsilon_{m-1} \\
& >\|S\|-\frac{1}{m} .
\end{aligned}
$$

Finally, in virtue of the previous lemma, $S^{*}$ attains its norm.

R Our proof is slightly different to the one presented by Zizler. In our case, we have taken $T^{*} \in L\left(X^{*}, Y^{*}\right)$, while he worked with a $\omega^{*}$-continuous operator. He had to prove that the operator obtained was $\omega^{*}$-continuous, something that is clear for us, as our operator is continuous with the topology of the norm. This idea has been extracted from [Rui94].

In fact, there is a result of Poliquin and Zizler, [PZ90, Theorem 1] that improves the previous theorem. They show that we can obtain an operator whose adjoint attains its norm and is so closed to the given operator that the difference between both of them is rank one.

From the Lindenstrauss-Zizler Theorem, we can easily see that, if $X$ is reflexive, we have

$$
N A(X, Y)=N A_{1}(X, Y)=N A_{2}(X, Y) .
$$

Then,

Corollary 2.2.28 If $X$ is a reflexive Banach space and $Y$ is an arbitrary Banach space, $N A(X, Y)$ is dense in $L(X, Y)$.

And in particular, when $X=Y$, we have a positive result for $\overline{N A(X)}=L(X)$ : 
Result 2.2.29 If $X$ is a reflexive Banach space,

$$
\overline{N A(X)}=L(X) \text {. }
$$




\subsection{SUMMARY}

POSitive ReSUlts FOR $N A(X, Y)=L(X, Y) \quad(\sharp X Y)$

\begin{tabular}{|c|c|c|c|}
\hline$X$ & $Y$ & RESULT & COMMENTARIES \\
\hline \hline Reflexive & $\mathbb{K}(\mathbb{R}$ or $\mathbb{C})$ & James th. & \\
\hline Reflexive & Finite dim. & Prop. 2.2.6 & $\begin{array}{c}\text { This result is a characterization of reflexivity : } \\
\text { if it holds for every finite dim. } Y \text { is reflexive }\end{array}$ \\
\hline Finite dim. & All & Prop. 2.2.8 & Direct consequence of the compactness of the ball \\
\hline Reflexive & $?$ & Prop. 2.2.10 & Necessary condition for $X$ \\
\hline
\end{tabular}

$(\sharp X Y)$ FOR $X$ AND $Y$ OF THE TYPE $C(S)$ OR $L^{p}(\mu)$

\begin{tabular}{|c|c|c|c|}
\hline$X$ & $Y$ & RESULT & COMMENTARIES \\
\hline \hline $\begin{array}{c}L^{p}(\mu), \mu \text { atomic } \\
\text { and } 1<p<\infty\end{array}$ & $\begin{array}{c}L^{r}(v), v \text { atomic } \\
\text { and } 1<r<\infty\end{array}$ & & \\
\cline { 1 - 2 } $\begin{array}{c}L^{p}(\mu), \\
1<p<\infty\end{array}$ & $\begin{array}{c}L^{r}(v), v \text { atomic } \\
\text { and } 1<r<2\end{array}$ & & This result is a characterization: If $X$ and $Y$ \\
\cline { 1 - 2 } $\begin{array}{c}L^{p}(\mu), \mu \text { atomic } \\
\text { and } 2<p<\infty\end{array}$ & $\begin{array}{c}L^{r}(v), \\
1<r<\infty\end{array}$ & \multirow{2}{*}{ Th. 2.2.17 } & are of the type $C(S)$ or $L^{p}(\mu)$ and verify \\
\cline { 1 - 2 } $\begin{array}{c}L^{p}(\mu), \\
1<p<\infty\end{array}$ & $\begin{array}{c}L^{r}(v), v \text { atomic } \\
\text { and } r=1\end{array}$ & & \multirow{2}{|c|}{$\sharp X Y)$, they belong to one of these five cases } \\
\cline { 1 - 2 } $\begin{array}{c}L^{p}(\mu), \mu \text { atomic } \\
\text { and } 2<p<\infty\end{array}$ & $\begin{aligned} L^{r}(v), \\
r=1\end{aligned}$ & & \\
\hline
\end{tabular}


POSITIVE RESUltS FOR $N A(X)=L(X) \quad(\sharp X)$

\begin{tabular}{|c|c|c|}
\hline$X$ & RESULT & COMMENTARIES \\
\hline \hline $\mathbb{K}(\mathbb{R}$ or $\mathbb{C})$ & James theorem & \\
\hline Finite dimensional & Proposition 2.2.6 & $\begin{array}{c}\text { Among the classical Banach spaces, } \\
\text { these are the only spaces verifying }(\sharp X)\end{array}$ \\
\hline
\end{tabular}

Positive Results fOr $\overline{N A(X, Y)}=L(X, Y) \quad(\overline{X Y})$

\begin{tabular}{|c|c|c|c|}
\hline$X$ & $Y$ & RESULT & COMMENTARIES \\
\hline \hline All & $\mathbb{K}(\mathbb{R}$ or $\mathbb{C})$ & Bishop-Phelps th. & \\
\hline Reflexive & Finite dim. & Proposition 2.2.6 & Particular case of $(\sharp X Y)$ \\
\hline Finite dim. & All & Proposition 2.2.8 & Particular case of $(\sharp X Y)$ \\
\hline$L^{p}(\mu)$ & $L^{r}(v)$ & Theorem 2.2.17 & 5 cases in the chart of $(\sharp X Y)$ \\
\hline Reflexive & All & Lindenstrauss-Zizler th. & \\
\hline
\end{tabular}

Positive Results fOr $\overline{N A(X)}=L(X) \quad(\bar{X})$

\begin{tabular}{|c|c|l|}
\hline$X$ & RESULT & COMMENTARIES \\
\hline \hline $\mathbb{K}(\mathbb{R}$ or $\mathbb{C})$ & James th. or Bishop-Phelps th. & Particular case of $(\sharp X)$ \\
\hline Finite dim. & Proposition 2.2.6 & Particular case of $(\sharp X)$ \\
\hline Reflexive & Lindenstrauss-Zizler th. & \\
\hline
\end{tabular}


NEGATIVE RESULTS FOR $\overline{N A(X, Y)}=L(X, Y) \quad(\overline{X Y})$

\begin{tabular}{|c|c|c|c|}
\hline$X$ & $Y$ & RESULT & COMMENTARIES \\
\hline \hline \multirow{2}{c}{$c_{0}$} & $\begin{array}{c}\text { Strictly convex space } \\
\text { such that there exists a } \\
\text { non-compact operator from } c_{0} \text { to } Y\end{array}$ & Proposition 2.2.21 & $\begin{array}{c}\text { The condition on } Y \text { is necessary to prove } \\
\text { the result from Proposition 2.2.21, } \\
\text { but is unknown to be necessary in general }\end{array}$ \\
\hline
\end{tabular}

Negative RESUltS FOR $\overline{N A(X)}=L(X) \quad(\bar{X})$

\begin{tabular}{|c|c|c|}
\hline$X$ & RESULT & COMMENTARIES \\
\hline \hline $\begin{array}{c}Y \oplus_{\infty} c_{0} \\
\text { with } Y \text { strictly convex }\end{array}$ & Proposition 2.2.22 & \\
\hline
\end{tabular}



LINDENSTRAUSS PROPERTIES "A" AND "B"

PROPERTIES $\alpha$ AND $\beta$

PARTINGTON AND SCHACHERMAYER THEOREMS

A SUFFICIENT CONDITION FOR RENORMING A BANACH SPACE WITH PROPERTY $\alpha$

PROPERTIES QUASI- $\alpha$ AND QUASI- $\beta$

BISHOP-PHELPS AND RADON-NIKODYM PROPERTIES

THE NON-LINEAR OPTIMIZATION PRINCIPLE OF BOURGAIN AND STEGALL

RELATION BETWEEN $\overline{N A(X)}=L(X)$ AND THE RNP

DENSITY OF NORM-ATTAINING OPERATORS BETWEEN $C(K)$ AND $C(L)$

C(K) SPACES WITH PROPERTY A

SUMMARY

\section{RESULTS ON NORM-ATTAINING OPERATORS}

In this chapter, we will expose some properties and known facts about operators which attain their norm and the density of this kind of operators in the set of bounded linear operators between two Banach spaces.

We will begin the chapter with the definition of properties $A$ and $B$. A Banach space $X$ has property $A$ (resp. $B$ ) if the set of norm-attaining operators from $X$ to $Y$ (resp. from $Y$ to $X$ ) is dense in $L(X, Y)$ for every Banach space $Y$. The presence of these two properties will be essential in the study of the problem of density of norm-attaining operators. However, we will notice that the problem of checking if a Banach space has one of these properties is really hard; this will lead to the appearance of properties $\alpha$ and $\beta$, geometric properties implying properties $A$ and $B$, respectively.

After presenting some spaces with properties $\alpha$ and $\beta$, we will prove that, indeed, $\beta$ implies $B$, and, with a slight modification, $\alpha$ implies $A$, but before that, we will have introduced the concept of uniformly strongly exposed points and proved that if the unit ball of a Banach space is the closed convex hull of a set of uniformly strongly exposed points, then the space has property A.

In the next section of the chapter, we will present Partington Theorem, which states that every Banach space can be renormed to satisfy property $\beta$, and the semi-analogous for property $\alpha$, Schachermayer Theorem, stating that every weakly compactly generated Banach space can be renormed with property $\alpha$. There is no complete duality between these two results, as there exist examples of Banach spaces that cannot be equivalently renormed to satisfy property $\alpha$. However, we will also show an improvement of Schachermayer Theorem, by Godun and Troyanski, where we can see that even though not every Banach space can be equivalently renormed with property $\alpha$, there is a really big class of spaces that admit this renorming.

Next, we will introduce property quasi- $\beta$, an intermediate property between $\beta$ and $B$, verifying that $\beta$ implies quasi- $\beta$ and quasi- $\beta$ implies $B$, and being stable under $c_{0}$-sums, something that property $\beta$ does not satisfy. Analogously, we will present property quasi- $\alpha$, whose dual property is quasi- $\beta$, implying $A$ and being implied by $\alpha$, which is stable by finite $\ell_{1}$-sums.

In the following section of this chapter, we reach what can be considered the most brilliant result in the theory of norm-attaining operators, the relation between the problem of density of 
norm-attaining operators and the Radon-Nikodym property. After presenting some definitions and important results, including Bourgain Theorem and the non-linear optimization principle of Bourgain and Stegall, we will conclude that a Banach space has the Radon-Nikodym property if, and only if, every space isomorphic to it has property $A$. This result is really important, as constitutes a link between the theory of norm-attaining operators and the field of measure theory. It might be used to provide positive and negative examples for one of those fields using the results of the other.

To continue with the relation between the theory of norm-attaining operators and the RadonNikodym property, we realise that, for a Banach space, the property that every space isomorphic to it has property $A$ is a very strong property, so we study the possibility of weaken this property to a property that only involves the space of the domain and still has an equivalence with the RNP. Our conjecture is that, for a Banach space $X$, having the RNP is equivalent to verifying $\overline{N A(X)}=L(X)$ for every equivalent renorming of $X$. We prove that if $X$ is isomorphic to a square of a Banach space, then this equivalence is true, and provide some positive and negative examples of spaces that are isomorphic to a square of a Banach space. Then, we show that the extension of the proof of the previous equivalence is still an open problem and give some steps to follow in order to try and prove this equivalence, a way that is not working yet.

Finally, we will study the specific case of the density of norm attaining operators between two spaces of the type $C(K)$. We will present some results to show that if $K, L$ are compact, Hausdorff topological spaces, then the set of norm-attaining operators between $C(K)$ and $C(L)$ is dense in the set of bounded linear ones. Later, we will see that $C(K)$ has property $A$ if, and only if, $K$ is finite, and we will also present some results of density for the set $N A(C(K), Y)$ when there exists a weakly compact operator from $C(K)$ to $Y$.

\subsection{LiNDENSTRAUSS PROPERTIES "A" AND "B"}

In his seminal paper [Lin63], Lindenstrauss considered that the problem $\overline{N A(X, Y)}=L(X, Y)$ is too general to admit a positive answer. For that reason, he proposed studying just one of the spaces $(X$ or $Y)$. Then, we may consider the two following properties.

\section{Definition 3.1.1 - Property A.}

We say that a Banach space $X$ has property $A$ if $N A(X, Y)$ is dense in $L(X, Y)$ for every Banach space $Y$.

\section{Definition 3.1.2- Property B.}

We say that a Banach space $Y$ has property $B$ if $N A(X, Y)$ is dense in $L(X, Y)$ for every Banach space $X$.

We have already seen some examples of spaces which have and do not have one of this properties.

\section{- Example 3.1.3}

1. $\mathbb{K}$ has property B. The Bishop-Phelps theorem shows that the norm-attaining functionals of a Banach space are dense, so, for every Banach space $X, \overline{N A(X, \mathbb{K})}=L(X, \mathbb{K})$. 
2. $c_{0}$ does not have property A. As seen in proposition 2.2.21, if $Y$ is a strictly convex Banach space and there is a non compact operator from $c_{0}$ to $Y, \overline{N A\left(c_{0}, Y\right)} \neq L\left(c_{0}, Y\right)$.

3. If $Y$ is strictly convex, isomorphic to $c_{0}, Y$ does not have property $\mathbf{B}$. It is also a consequence of proposition 2.2.21.

4. If $X$ is a reflexive Banach space, $X$ has property A. We have seen this in corollary 2.2.28, as a consequence of the Lindenstrauss-Zizler Theorem.

5. $X=c_{0} \oplus_{\infty} Y$, with $Y$ strictly convex, isomorphic to $c_{0}$, does not have either property A nor property B. This is shown in proposition 2.2.22.

Now, it is clear from the definition of properties $A$ and $B$ that if a Banach space $X$ has one of this properties, in particular $\overline{N A(X)}=L(X)$. Then,

Result 3.1.4 Let $X$ be a Banach space verifying property A or property B. Then,

$$
\overline{N A(X)}=L(X) \text {. }
$$

\subsubsection{PROPERTIES $\alpha$ AND $\beta$}

In this section, we will study some results of Lindenstrauss that give sufficient conditions for a Banach space to have properties A or B. For that, we will define two new properties of geometric nature, properties $\alpha$ and $\beta$. The advantage found in these properties with respect to properties $A$ and $B$ is that they are defined intrinsecally, so they apparently do not involve the set of normattaining operators. They are also very general from the isomorphic point of view, as every Banach space can be equivalently renormed to satisfy property $\beta$ and many Banach spaces can be equivalently renormed to satisfy property $\alpha$. We will see these results in the following sections.

\section{Definition 3.1.5 - PROPERTY $\alpha$.}

We say that a Banach space $X$ has property $\alpha$ if there exist $\left\{x_{\lambda}: \lambda \in \Lambda\right\}$ and $\left\{x_{\lambda}^{*}: \lambda \in \Lambda\right\}$, subsets of $X$ and $X^{*}$, respectively, such that

1) $\left\|x_{\lambda}\right\|=\left\|x_{\lambda}^{*}\right\|=x_{\lambda}^{*}\left(x_{\lambda}\right)=1 \quad \forall \lambda \in \Lambda$.

2) There exists a constant $\rho$ with $0 \leq \rho<1$ such that, for $\lambda, \mu \in \Lambda$ with $\lambda \neq \mu$, we have

$$
\left|x_{\lambda}^{*}\left(x_{\mu}\right)\right| \leq \rho .
$$

3) The absolute convex hull of the set $\left\{x_{\lambda}: \lambda \in \Lambda\right\}$ is dense in the unit ball of $X$.

\section{Definition 3.1.6- PROPERTY $\beta$.}

We say that a Banach space $Y$ has property $\beta$ when there exist $\left\{y_{\lambda}: \lambda \in \Lambda\right\}$ and $\left\{y_{\lambda}^{*}: \lambda \in \Lambda\right\}$, subsets of $Y$ and $Y^{*}$, respectively, such that

1) $\left\|y_{\lambda}\right\|=\left\|y_{\lambda}^{*}\right\|=y_{\lambda}^{*}\left(y_{\lambda}\right)=1 \quad \forall \lambda \in \Lambda$.

2) There exists a constant $\rho$ with $0 \leq \rho<1$ such that, for $\lambda, \mu \in \Lambda$ with $\lambda \neq \mu$, we have

$$
\left|y_{\lambda}^{*}\left(y_{\mu}\right)\right| \leq \rho .
$$


3) $\|y\|=\operatorname{Sup}\left\{\left|y_{\lambda}^{*}(y)\right|: \lambda \in \Lambda\right\}, \quad \forall y \in Y$.

R If $Y$ is an arbitrary Banach space with property $\beta, X$ another arbitrary Banach space and $T \in L(X, Y)$, then we can deduce from the third condition of the definition of property $\beta$ that

$$
\|T\|=\operatorname{Sup}\left\{\left\|T^{*} y_{\lambda}^{*}\right\|: \lambda \in \Lambda\right\}
$$

Notation 3.1. When the value of the constant $\rho$ in the definition of property $\alpha$ (resp. property $\beta$ ) is relevant, we will say that the space has property $(\alpha, \rho)$ (resp. property $(\beta, \rho)$ ).

From an intuitive point of view, spaces with property $\alpha$ look as $\ell_{1}$ and spaces with property $\beta$ look as $\ell_{\infty}$. Indeed, we will show now that these spaces verify these properties, respectively.

\section{- Example 3.1.7}

The space $\ell_{1}$ has property $\alpha$. Consider $\mathbb{N}$ as the set of indices $\Lambda$, the constant $\rho=0$ and the set

$$
\left\{\left(e_{n}, e_{n}^{*}\right): n \in \mathbb{N}\right\} \subseteq \mathbb{S}_{\ell_{1}} \times \mathbb{S}_{\ell_{\infty}},
$$

where $\left\{e_{n}: n \in \mathbb{N}\right\}$ is the canonical base of $\ell_{1}$ and $\left\{e_{n}^{*}: n \in \mathbb{N}\right\}$ is the set of functionals associated to that base. Then, it is clear that these sets verify the conditions on the definition of property $\alpha$.

Now, we will present some spaces with property $\beta$.

\section{- Example 3.1.8}

Let $\Lambda$ be an arbitrary, non-empty set and let $\ell_{\infty}^{\Lambda}$ be the Banach space of all the bounded functions from $\Lambda$ to $\mathbb{K}$, with the usual norm:

$$
\|y\|_{\infty}=\operatorname{Sup}\{|y(\lambda)|: \lambda \in \Lambda\} \quad\left(y \in \ell_{\infty}^{\Lambda}\right) .
$$

For every $\lambda \in \Lambda$, let $y_{\lambda} \in \ell_{\infty}^{\Lambda}$ the characteristic function of the set $\{\lambda\}$ and let $y_{\lambda}^{*} \in\left(\ell_{\infty}^{\Lambda}\right)^{*}$ the functional that evaluates in point $\lambda$. Then, it is easy to see that the sets $\left\{y_{\lambda}: \lambda \in \Lambda\right\}$ and $\left\{y_{\lambda}^{*}: \lambda \in \Lambda\right\}$ verify the three conditions in property $\beta$ (the second one with $\rho=0$ ). Hence, $\ell_{\infty}^{\Lambda}$ verifies property $\beta$.

Now, let $X$ be a closed subspace of $\ell_{\infty}^{\Lambda}$ containing $c_{0}^{\Lambda} .{ }^{1}$ Then, $\left\{y_{\lambda}: \lambda \in \Lambda\right\} \subset X$ and if we change every functional $y_{\lambda}^{*}$ for their restriction to $X$, the same conditions are still verified (in particular, the condition $\left\{y_{\lambda}: \lambda \in \Lambda\right\} \subset X$ implies that the norm of $y_{\lambda}^{*}$ does not decrease by restricting that to $X$ ). This implies that $X$ has property $\beta$.

In particular, the spaces $c_{0}, c$ and $\ell_{\infty}$ have property $\beta$.

Reciprocally, if there is a Banach space $Y$ with property $\beta$ and the constant $\rho$ appearing in the second condition of the definition is null, then there exists a set $\Lambda$ such that $Y$ is isometrically isomorphic to a closed subspace of $\ell_{\infty}^{\Lambda}$ containing $c_{0}^{\Lambda}$.

\footnotetext{
${ }^{1}$ Remember that $y \in c_{0}^{\Lambda}$ if the set $\{\lambda \in \Lambda:|y(\lambda)| \geq \varepsilon\}$ is finite for every $\varepsilon>0$.
} 


\section{- Example 3.1.9}

For a real, finite dimensional space, we can see that it has property $\beta$ if, and only if, its unit ball is a polyhedron (finite intersection of closed subspaces).

In fact, we have the following result for finite dimensional spaces, whose proof we can find in [Agu98].

Proposition 3.1.10 Let $Y$ be a finite dimensional normed space. The following statements are equivalent:

1. $Y$ has property $\beta$.

2. The set $\operatorname{Ext}\left(\mathbb{B}_{Y^{*}}\right)$ of the extreme points of $\mathbb{B}_{Y^{*}}$ is finite, up to rotations. More specifically, there exists a finite set $F \subset Y^{*}$ such that $\operatorname{Ext}\left(\mathbb{B}_{Y^{*}}\right)=\mathbb{T} F$, where $\mathbb{T}$ is the set of scalars with module 1 , i.e., $\mathbb{T}=\{\lambda \in \mathbb{K}:|\lambda|=1\}$.

In the real case, this characterization can be interpreted as we have mentioned above: A finite dimensional, real, normed space has property $\beta$ if, and only if, its unit ball is a polyhedron (finite intersection of closed semispaces).

\section{- Example 3.1.11}

Let $L$ be a topological space with a dense subset of isolated points. We denote by $C_{b}(L)$ the Banach space of all continuous bounded functions in $L$. Then, $C_{b}(L)$, with its usual norm, has property $\beta$

For the proof, let us consider $\left\{x_{\lambda}: \lambda \in \Lambda\right\}$ the dense subset of isolated points of $L$. Then, consider the functions

$$
y_{\lambda}(x)=\left\{\begin{array}{l}
1, \text { if } x=x_{\lambda} \\
0, \text { if } x \neq x_{\lambda}
\end{array} \quad(x \in L, \lambda \in \Lambda)\right.
$$

and the evaluation functionals

$$
y_{\lambda}^{*}(y)=y\left(x_{\lambda}\right) \quad\left(y \in C_{b}(L), \lambda \in \Lambda\right) .
$$

Then, the sets $\left\{y_{\lambda}: \lambda \in \Lambda\right\}$ and $\left\{y_{\lambda}^{*}: \lambda \in \Lambda\right\}$ verify property $\beta$ with $\rho=0$.

Now, we can enunciate and demonstrate the following sufficient condition for property $B$, proved by Lindenstrauss in [Lin63].

Theorem 3.1.12 Property $\beta$ implies property $B$.

Proof: Let $Y$ be a Banach space with property $\beta$ and denote by $\left\{y_{\lambda}: \lambda \in \Lambda\right\}$ and $\left\{y_{\lambda}^{*}: \lambda \in \Lambda\right\}$, respectively, the subsets of $Y^{*}$ that verify the conditions in the definition. We consider $T \in$ $L(X, Y)$. Then, by the third condition in the definition, it is easy to see that

$$
\|T\|=\operatorname{Sup}_{\lambda \in \Lambda}\left\{\left\|T^{*} y_{\lambda}^{*}\right\|\right\}
$$


Moreover, if the previous supremum is a maximum, i.e., there exists $\alpha \in \Lambda$ such that $\|T\|=\left\|T^{*} y_{\alpha}^{*}\right\|$, and this functional attains its norm, then $T$ also attains its norm.

Now, if we consider an arbitrary operator $T_{0}$, we want to perturb it in order to get another operator $T$ which attains its norm. Without loss of generality, we take $\left\|T_{0}\right\|=1$. Then, given $\varepsilon>0$, we take $\alpha \in \Lambda$ so that

$$
\left\|T_{0}^{*} y_{\alpha}^{*}\right\| \geq 1-\delta
$$

where $\delta>0$ is a positive number to be determined in function of $\varepsilon$. Now, using Bishop-Phelps theorem, there exists $g \in X^{*}$, a norm-attaining functional, which verifies,

$$
\|g\|=\left\|T_{0}^{*} y_{\alpha}^{*}\right\| \text { and }\left\|g-T_{0}^{*} y_{\alpha}^{*}\right\|<\delta .
$$

Then, we define,

$$
T(x)=T_{0}(x)+\left[\left(1+\frac{\varepsilon}{2}\right) g(x)-y_{\alpha}^{*}\left(T_{0} x\right)\right] y_{\alpha} .
$$

And we have

$$
\left\|T-T_{0}\right\| \leq \frac{\varepsilon}{2}\|g\|+\left\|g-T_{0}^{*} y_{\alpha}^{*}\right\|<\frac{\varepsilon}{2}+\delta<\varepsilon .
$$

So we take, from the beginning, $\delta<\frac{\varepsilon}{2}$. Now we check that $T$ attains its norm. For $\lambda \in \Lambda$, with $\lambda \neq \alpha$, we have:

$$
\left\|T^{*} y_{\lambda}^{*}\right\| \leq\left\|T_{0}\right\|+\left|y_{\lambda}^{*}\left(y_{\alpha}\right)\right|\left|\frac{\varepsilon}{2}\|g\|+\left\|T_{0}^{*} y_{\alpha}^{*}-g\right\|\right| \leq 1+\rho\left(\frac{\varepsilon}{2}+\delta\right)<1+\rho \varepsilon,
$$

where $\rho$ is the constant from the second condition of the definition of property $\beta$.

On the other hand, for $y_{\alpha}$, we have

$$
T^{*} y_{\alpha}^{*}(x)=y_{\alpha}^{*} T(x)=y_{\alpha}^{*} T_{0}(x)+\left[\left(1+\frac{\varepsilon}{2}\right) g(x)-y_{\alpha}^{*}\left(T_{0} x\right)\right] y_{\alpha}^{*}\left(y_{\alpha}\right) \stackrel{1}{=}\left(1+\frac{\varepsilon}{2}\right) g(x) .
$$

Hence,

$$
T^{*} y_{\alpha}^{*}=\left(1+\frac{\varepsilon}{2}\right) g \quad \text { and } \quad\left\|T^{*} y_{\alpha}^{*}\right\| \geq\left(1+\frac{\varepsilon}{2}\right)(1-\delta)
$$

Then, if we take $0<\delta<\frac{\varepsilon}{2}$ so that $\left(1+\frac{\varepsilon}{2}\right)(1-\delta)>1+\rho\left(\frac{\varepsilon}{2}+\delta\right)$ (something possible, because if we take $\delta \rightarrow 0$, the first term tends to $1+\frac{\varepsilon}{2}$ and the second one to $1+\rho \frac{\varepsilon}{2}<1+\frac{\varepsilon}{2}$ ), we have $\left\|T^{*} y_{\lambda}^{*}\right\| \leq\left\|T^{*} y_{\alpha}^{*}\right\|$ for every $\lambda \in \Lambda$. And $T^{*} y_{\alpha}^{*}$ attains its norm, because it is a multiple of $g$, which attains its norm by hypothesis.

Consequently, $T$ attains its norm.

We have just proved that property $\beta$ implies property $B$. The reciprocal is not true. For that, see Example 3.1.42, where we present an example of space with property quasi- $\beta$ (which implies property $B$ ) and lacking property $\beta$.

It is also important to make the following remark about property $B$. 
R Property $B$ is not stable under isomorphisms. One example of this is the space $c_{0}$. We have seen in this section that $c_{0}$ has property $\beta$, so it has property $B$. But we showed in the previous section the example of Lindenstrauss of a space, isomorphic to $c_{0}$, without property $B$.

Now, we are going to study a sufficient condition for property $A$, also given by Lindenstrauss. For that, we need the following definition:

Definition 3.1.13 - UNIFORMLY STRONGLY EXPOSED POINTS.

Let $E=\left\{x_{\lambda}: \lambda \in \Lambda\right\}$ be a subset of the unit sphere of a Banach space $X$. We say that $E$ is a set of uniformly strongly exposed points (u.s.e.) if there exists a subset $\left\{x_{\lambda}^{*}: \lambda \in \Lambda\right\}$ in the unit sphere of $X^{*}$ verifying:

1) $x_{\lambda}^{*}\left(x_{\lambda}\right)=1 \quad \forall \lambda \in \Lambda$.

2) For every $\varepsilon>0$, we can find a $\delta>0$ such that, if $\lambda \in \Lambda$ and $x \in \mathbb{S}_{X}$ verifies $\operatorname{Re} x_{\lambda}^{*}(x)>$ $1-\delta$, then $\left\|x-x_{\lambda}\right\|<\varepsilon$.

In a uniformly convex space, the set of all the boundary points of the unit ball is u.s.e. Also, the set of all the extreme points of the unit ball of $\ell_{1}$ is u.s.e.

Theorem 3.1.14 Let $X$ be a Banach space whose unit ball is the closed convex hull of a set of uniformly strongly exposed points $E \subset \mathbb{S}_{X}$. Then, $X$ has property $A$.

The proof of this result is very similar to the proof of the previous theorem, and can be found in [Lin63].

It is quite easy to give an example of a bidimensional space whose unit ball is not the closed convex hull of a set of uniformly strongly exposed points. In particular, a reflexive space might not verify the hypothesis in the previous theorem.

- Example 3.1.15 Consider the space $X=\mathbb{R}^{2}$ with the norm $\|\cdot\|$ whose unit ball is the set $A=\mathbb{B}_{2}+\mathbb{B}_{\infty} \subset \mathbb{R}^{2}$, where $\mathbb{B}_{2}$ and $\mathbb{B}_{\infty}$ are the unit balls for the euclidean and maximum norm, respectively.

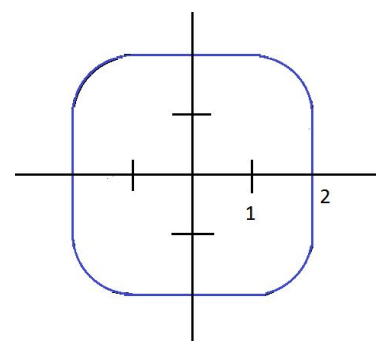

Then, it is easy to compute the norm of $X^{*}$. If $x^{*} \in X^{*}$,

$$
\begin{aligned}
\left\|x^{*}\right\| & =\sup \left\{\left|x^{*}(u+v)\right|: u \in \mathbb{B}_{2}, v \in \mathbb{B}_{\infty}\right\} \\
& =\sup \left\{\left|x^{*}(u)\right|: u \in \mathbb{B}_{2}\right\}+\sup \left\{\left|x^{*}(v)\right|: v \in \mathbb{B}_{\infty}\right\} \\
& =\left\|x^{*}\right\|_{2}+\left\|x^{*}\right\|_{1}
\end{aligned}
$$

Moreover, it is clear that if $x \in \mathbb{S}_{X}$ and $x^{*} \in \mathbb{S}_{X^{*}}$ is a functional such that $x^{*}(x)=1$, taking $x=u+v$, with $u \in \mathbb{B}_{2}$ and $v \in \mathbb{B}_{\infty}$, we have 


$$
x^{*}(u)=\left\|x^{*}\right\|_{2} \text { and } x^{*}(v)=\left\|x^{*}\right\|_{1} .
$$

If $E \subseteq \mathbb{S}_{X}$ is a set of uniformly strongly exposed points, then it only contains extreme points of $\mathbb{B}_{X}$, so

$$
E \subseteq\{(\cos \theta, \operatorname{sen} \theta)+( \pm 1, \pm 1): 0 \leq \theta \leq 2 \pi\} .
$$

As $E \cup-E \subseteq \mathbb{S}_{X}$ is uniformly strongly exposed too, we can suppose that $E=-E$. Then, if $(2,0) \in \mathbb{B}_{X}$ belongs to the closure of the absolutely convex hull of $E$, then there exists a sequence $\left\{\theta_{n}\right\}_{n \in \mathbb{N}} \rightarrow 0$, with $0 \leq \theta_{n} \leq \pi / 2 \quad \forall n \in \mathbb{N}$; such that

$$
y_{n}:=(\cos \theta, \operatorname{sen} \theta)+(1,1) \in E \quad \forall n \in \mathbb{N} .
$$

If $\left\{y_{n}^{*}: n \in \mathbb{N}\right\}$ is the family of functionals which exposes strongly $\left\{y_{n}: n \in \mathbb{N}\right\}$, then it must be necessarily

$$
y_{n}^{*}=\left(\frac{\cos \theta_{n}}{1+\operatorname{sen} \theta_{n}+\cos \theta_{n}}, \frac{\operatorname{sen} \theta_{n}}{1+\operatorname{sen} \theta_{n}+\cos \theta_{n}}\right) \quad(n \in \mathbb{N}) .
$$

Hence,

$$
y_{n}^{*}(2,0)=\frac{2 \cos \theta_{n}}{1+\operatorname{sen} \theta_{n}+\cos \theta_{n}} \rightarrow 1
$$

while

$$
\left\|(2,0)-y_{n}\right\|=\left\|\left(-1+\cos \theta_{n}, 1+\operatorname{sen} \theta_{n}\right)\right\| \rightarrow 1 .
$$

Therefore, $E$ is not a set a uniformly strongly exposed points.

Reciprocally, it is easy to see that the space $\ell_{1}$ verifies the hypothesis in the previous theorem by taking its canonical basis as the set $E$. For that reason, the sufficient conditions given by this theorem and those given in Corollary 2.2.28, for a Banach space to have property $A$, are independent.

In fact, there exists a pseudoreciprocal result for Theorem 3.1.14 in the separable case, also due to Lindenstrauss [Lin63, Theorem 2.2]. It is the following one.

Proposition 3.1.16 Let $X$ be a separable Banach space verifying property $A$. Then, the unit ball of $X$ is the absolutely closed convex hull of its strongly exposed points.

If we look at Theorems 3.1.12 and 3.1.14, we can observe that there is a certain asymmetry between the sufficient conditions given for property $B$ and $A$, respectively. This asymmetry is corrected by introducing the concept of property $\alpha$ and proving the next theorem, due to $\mathrm{W}$. Schachermayer [Sch83a].

Theorem 3.1.17 Property $\alpha$ implies property $A$.

Proof: We can give an easy, direct proof of this theorem, whose paralelism with Theorem 3.1.12 is clear. Let us suppose that $X$ verifies property $\alpha, Y$ is an arbitrary Banach space, $T \in L(X, Y)$ satisfies $\|T\|=1$ and $\varepsilon>0$. Then, we take 


$$
S(x)=T(x)+\frac{\varepsilon}{2} x_{\alpha}^{*}(x) T x_{\alpha},
$$

where $\alpha \in \Lambda$ is chosen so that

$$
\left\|T x_{\alpha}\right\|<1-\delta
$$

for suitable $\delta>0$. For the definition of $S$, it is clear that $\|S-T\| \leq \frac{\varepsilon}{2}$. And for $\lambda \neq \alpha$, as $\left|x_{\alpha}^{*}\left(x_{\lambda}\right)\right| \leq \rho$, we have

$$
\left\|S x_{\lambda}\right\| \leq 1+\rho \frac{\varepsilon}{2}
$$

while

$$
\left\|S x_{\alpha}\right\|=\left(1+\frac{\varepsilon}{2}\right)\left\|T x_{\alpha}\right\|>\left(1+\frac{\varepsilon}{2}\right)(1-\delta),
$$

and if we choose $\delta$ so that $\left(1+\frac{\varepsilon}{2}\right)(1-\delta)>1+\rho \frac{\varepsilon}{2}$, we obtain

$$
\|S\|=\operatorname{Sup}\left\{\left\|S x_{\lambda}\right\|: \lambda \in \Lambda\right\}=\left\|S x_{\alpha}\right\| .
$$

Therefore, $S$ attains its norm.

We are now in a position to give some new positive answers for the problem $\overline{N A(X)}=L(X)$. At the beginning of this subsection, we presented some examples of spaces with property $\beta$. Then, we proved in Theorem 3.1.12 that $\beta$ implies $B$. And finally, in Result 3.1.4, we saw that if a Banach space has property $B$, then we have a positive answer for our problem. For that, we can present the next particular result.

Result 3.1.18 Let $X$ be $c_{0}, c$ or $\ell_{\infty}$. Then,

$$
\overline{N A(X)}=L(X)
$$

Besides from this specific result, due to Theorem 3.1.12 and Theorem 3.1.17, it is clear that, in general,

Result 3.1.19 Let $X$ be a Banach space with property $\alpha$ or property $\beta$. Then,

$$
\overline{N A(X)}=L(X) \text {. }
$$

In the following sections, we will try to find examples of spaces with any of these properties.

\subsubsection{PARTINGton AND SCHAChermayer theOREMS}

The results in the previous section come mainly from the work of Lindenstrauss. We consider now the problem of density of the set of norm-attaining operators from an isomorphic point of view, i.e., we are going to think which spaces can be equivalently renormed in order to verify properties $A$ or $B$.

We already know that property $B$ is not stable under isomorphisms. In fact, J. Partington proved in [Par82] that we can obtain a completely satisfactory result for property $B$ from the isomorphic point of view: 


\section{Theorem 3.1.20 - PARTINGTON.}

Every Banach space can be renormed to satisfy property $\beta$ (and, consequently, property $B$ ).

In fact, Partington shows that if $(X,\|\cdot\|)$ is an arbitrary Banach space, and $K>3$, there exists an equivalent norm $\||\cdot|\|$ in $X$, so that $(X, \||\cdot| \mid)$ has property $\beta$ and

$$
\|x\| \leq\|x\|\|\leq K\| x \| \quad(x \in X) .
$$

It is easy to check that if $Y$ is a Banach space that verifies property $\beta$ and its dimension is greater or equal than two, then $Y$ is not strictly convex. In fact, the family of points lying in the relative interior of a face of the unit sphere is dense (by a face we mean the intersection of an hyperplane with $\mathbb{S}$, provided its relative interior to $\mathbb{S}$ is non-empty) [Mor97].

Moreover, there is no known example of a strictly convex Banach space with dimension greater than one with property $B$. This is an important key for Chapter 4 , where we will present some counterexamples for the problem of density of the set of norm-attaining operators.

For property $A$, the question about the stability of the property under isomorphisms has been an open question for many years. It was answered partially in 1983, by W. Schachermayer, who proved the following result in [Sch83a], with the help of the construction used by Partington in the previous theorem. Before enunciating this result, we have to give a previous definition.

\section{Definition 3.1.21 - WeAKLY COMPACtLY GENERATED.}

Let $X$ be a Banach space. We say that $X$ is weakly compactly generated (W.C.G.) if there exists a subset $K$ of $X$ which is weakly compact and such that

$$
X=\overline{\operatorname{span}(K)} \text {. }
$$

Now we can enunciate the following result.

\section{Theorem 3.1.22 - SCHACHERMAYER.}

Every weakly compactly generated Banach space can be renormed with property $\alpha$ (and, consequently, property $A$ ).

We are not going to prove here any of these two results. However, we are going to show the relation of the Schachermayer theorem with the Partington one. It comes from an elemental observation.

The hypothesis in the Schachermayer theorem is that a certain Banach space $X$ has property $\alpha$. Then, there exist $\left\{x_{\lambda}: \lambda \in \Lambda\right\}$ and $\left\{x_{\lambda}^{*}: \lambda \in \Lambda\right\}$, subsets of $X$ and $X^{*}$, respectively, such that

1) $\left\|x_{\lambda}\right\|=\left\|x_{\lambda}^{*}\right\|=x_{\lambda}^{*}\left(x_{\lambda}\right)=1 \quad \forall \lambda \in \Lambda$.

2) There exists a constant $\rho$ with $0 \leq \rho<1$ such that, for $\lambda, \mu \in \Lambda$ with $\lambda \neq \mu$, we have

$$
\left|x_{\lambda}^{*}\left(x_{\mu}\right)\right| \leq \rho .
$$

3) The absolute convex hull of the set $\left\{x_{\lambda}: \lambda \in \Lambda\right\}$ is dense in the unit ball of $X$.

Then, considering each $x_{\lambda}$ as an element in $X^{* *}$, the third condition says that:

$$
\left\|x^{*}\right\|=\operatorname{Sup}\left\{\left|x_{\lambda}\left(x^{*}\right)\right|: \lambda \in \Lambda\right\} \quad\left(x^{*} \in X^{*}\right) .
$$


and we clearly have that $X^{*}$ verifies property $\beta$.

Reciprocally, if $X^{*}$ has property $\beta$ and the functionals appearing in the definition of this property can be taken from $X$ (because, in general, we only know that they belong to $X^{* *}$ ), then $X$ has property $\alpha$.

Consequently, there is a partial duality between properties $\alpha$ and $\beta$. In fact, the duality is complete for reflexive spaces. The following result appears in [Sch83a] too.

Proposition 3.1.23 A reflexive Banach space $X$ has property $\alpha$ (respectively, $\beta$ ) if, and only if, $X^{*}$ verifies property $\beta$ (resp., $\alpha$ ).

And as an immediate consequence of this proposition, we have:

Corollary 3.1.24 Every reflexive Banach space can be renormed with property $\alpha$.

However, from the point of view of norm-attaining operators, this corollary lacks of interest, as we already knew that a reflexive space always verifies property $A$.

From the theorems of Partington and Schachermayer, we can obtain some more information about the problem we are studying, $\overline{N A(X)}=L(X)$. In fact, as a consequence of the Partington theorem, we have the following result.

Result 3.1.25 Every Banach space can be renormed to a space $X$ that satisfies

$$
\overline{N A(X)}=L(X) \text {. }
$$

As a consequence of the Schachermayer theorem, we would just obtain that every weakly compactly generated Banach space can be renormed to verify $\overline{N A(X)}=L(X)$, which is, clearly, a weaker condition than the one in the previous result.

Now, if $X$ is an arbitrary Banach space and we want to renorm it with property $\alpha$, it seems natural to renorm $X^{*}$ with property $\beta$ using the Partington theorem. The only problem is that we are not sure that the elements of $X^{* *}$ actually belong to $X$, so we do not know if the new norm in $X^{*}$ is, in fact, a dual norm.

However, Schachermayer succeeded renorming any weakly compactly generated space to have property $\alpha$. The reflexive case is, as we have seen, a direct consequence of the Partington theorem.

Another interesting case are the separable spaces. For $c_{0}$, we can prove the following proposition, which appears in [Pay93].

Proposition 3.1.26 For $K>1$, there exists a norm $|\| \cdot|||$ in $c_{0}$ such that

$$
\||| x|\||\leq\|x\| \leq K|\| x|\| \mid \quad\left(x \in c_{0}\right),
$$

where $\|\cdot\|$ is the usual norm of $c_{0}$ and $\left(c_{0},\||\cdot|\|\right)$ verifies property $\alpha$ (so, also, property $A$ ).

This proposition implies an important result. We have already seen that $c_{0}$, with its usual norm, does not have property $A$. However, in this proposition we see that we can find a norm that makes $c_{0}$ have property $A$. Then, 
Corollary 3.1.27 Property $A$ is not stable under isomorphisms.

And also, as $\ell_{1}$ is the dual of $c_{0}$,

Corollary 3.1.28 For $K>1$, there exists a norm $\||\cdot|||$ in $\ell_{1}$ such that

$$
\left\|| | x \left|\| \leq \| x \left\|\leq K|\|x\|| \quad\left(x \in \ell_{1}\right),\right.\right.\right.
$$

where $\|\cdot\|$ is the usual norm of $\ell_{1}$ and $\left(\ell_{1}, \||\cdot|||\right)$ verifies property $\beta$ (so, also, property $B$ ).

\subsubsection{A SUFFICIENT CONDITION FOR RENORMING A BANACH SPACE WITH PROPERTY $\alpha$}

In the previous section, we have shown two important results about renorming spaces. Isomorphically, we know from the Partington theorem (Theorem 3.1.20) that every Banach space can be renormed with property $\beta$ (implying property $B$ ). Hence, for every Banach space there is another one isomorphic to it verifying property $B$.

However, Schachermayer's result (Theorem 3.1.22) only guarantees the symmetric condition (i.e., that every Banach space can be renormed with property $\alpha$ ) when the space is weakly compactly generated. Some years after this result, Godun and Troyanski published in [GT93] a better result for the existence of spaces which can be renormed with property $\alpha$. We are going to show this result as it appears in [Háj+07]; but before enunciating it, we have to introduce some new concepts.

Firstly, we will introduce the concepts of biorthogonal system and fundamental biorthogonal system.

Definition 3.1.29 - BIORTHOGONAL SYSTEM.

Let $X$ be a Banach space. Let $\Lambda$ be a nonempty set. A family $\left\{\left(x_{\lambda}, x_{\lambda}^{*}\right)\right\}_{\lambda \in \Lambda}$ of pairs in $X \times X^{*}$ is called a biorthogonal system in $X \times X^{*}$ if $x_{\beta}^{*}\left(x_{\alpha}\right)=\delta_{\alpha, \beta}$, where $\delta_{\alpha, \beta}$ is the Kronecker delta, $\forall \alpha, \beta \in \Lambda$.

\section{Definition 3.1.30 - FUNDAMENTAL BIORTHOgONAL SYSTEM.}

Let $X$ be a Banach space. A family $\left\{x_{\lambda}\right\}_{\lambda \in \Lambda}$ of vectors in the Banach space $X$ is called fundamental if $\overline{\operatorname{span}}\left\{x_{\lambda}\right\}_{\lambda \in \Lambda}=X$.

In the case of a fundamental minimal system $\left\{x_{\lambda}\right\}_{\lambda \in \Lambda}$ in $X$, there exists a unique system $\left\{x_{\lambda}^{*}\right\}_{\lambda \in \Lambda}$ (its system of functional coefficients) in $X^{*}$ such that $\left\{\left(x_{\lambda}, x_{\lambda}^{*}\right)\right\}_{\lambda \in \Lambda}$ is a biorthogonal system, what we also call fundamental biorthogonal system.

Notation 3.2. We will use the abbreviated notation $\left\{x_{\lambda}\right\}_{\lambda \in \Lambda}$ in the case of a fundamental biorthogonal system $\left\{\left(x_{\lambda}, x_{\lambda}^{*}\right)\right\}_{\lambda \in \Lambda^{*}}$.

In the first result about fundamental biorthogonal systems we are going to show, we need the concept of long Schauder basis. For that, we will show how to define the sum of a transfinite sequence of vectors. 
Definition 3.1.31 Let $\Gamma$ be an ordinal and $\left\{x_{\gamma}\right\}_{\gamma=0}^{\Gamma}:=\left\{x_{\gamma}: 0 \leq \gamma<\Gamma\right\}$ be a transfinite sequence of vectors from $X$. We put $x=\sum_{\gamma=0}^{\Gamma} x_{\gamma}$ to be the sum of the series of the elements $\left\{x_{\gamma}\right\}_{\gamma=0}^{\Gamma}$ (and the series is called convergent) if there exists a continuous function $S:[1, \Gamma] \rightarrow X$, where $[1, \Gamma]$ is equipped with the order topology, such that

$$
S(1)=x_{0}, \quad S(\Gamma)=x, \quad S(\gamma+1)=S(\gamma)+x_{\gamma} \text { for } \gamma<\Gamma .
$$

R It is clear that if $\Gamma=\omega$ this definition coincides with the usual definition of convergence of a series.

\section{Definition 3.1.32 - LONG SCHAUDER BASIS.}

A Schauder basis of a Banach space $X$ is a sequence $\left\{v_{n}\right\}$ of elements of $X$ such that for every element $x \in X$ there exists a unique sequence $\left\{c_{n}\right\}$ of scalars so that

$$
x=\sum_{n=0}^{\infty} c_{n} v_{n}
$$

where the convergence is understood with respect to the norm topology

$$
\lim _{n \rightarrow \infty}\left\|x-\sum_{k=0}^{n} c_{k} v_{k}\right\|=0 .
$$

A transfinite sequence $\left\{v_{\gamma}\right\}_{\gamma=0}^{\Gamma}$ of vectors from a Banach space $X$ is called a long (or transfinite) Schauder basis if for every $x \in X$ there exists a unique transfinite sequence $\left\{c_{\gamma}\right\}_{\gamma=0}^{\Gamma}$ of scalars so that

$$
x=\sum_{\gamma=0}^{\Gamma} c_{\gamma} v_{\gamma} .
$$

If $\left\{v_{\gamma}\right\}_{\gamma=0}^{\Gamma}$ is a long Schauder basis of a Banach space $X$, then the canonical projections $P_{\alpha}: X \rightarrow X$ are defined for $1 \leq \alpha<\Gamma$ by

$$
P_{\alpha}\left(\sum_{\gamma=0}^{\Gamma} c_{\gamma} v_{\gamma}\right):=\sum_{\gamma=0}^{\alpha} c_{\gamma} v_{\gamma} .
$$

Lemma 3.1.33 Let $\left\{v_{\gamma}\right\}_{\gamma=0}^{\Gamma}$ be a long Schauder basis of a Banach space $X$. The canonical projections $P_{\alpha}$ satisfy

1. $\operatorname{dim}\left(\left(P_{\alpha+1}-P_{\alpha}\right)(X)\right)=1, \alpha<\Gamma$

2. $P_{\alpha} P_{\beta}=P_{\beta} P_{\alpha}=P_{\min (\alpha, \beta)}$

3. $P_{\alpha}(x)=\lim _{\gamma \rightarrow \alpha} P_{\gamma}(x)$ if $\alpha$ is a limit ordinal, and $\lim _{\alpha \rightarrow \Gamma} P_{\alpha}(x)=x$ for every $x \in X$.

The value bc $\left\{v_{\gamma}\right\}_{\gamma=0}^{\Gamma}=\sup _{\gamma<\Gamma}\left\|P_{\gamma}\right\|$ is called the basis constant of $\left\{v_{\gamma}\right\}_{\gamma=0}^{\Gamma}$. Considering the vectors $v_{\gamma}$, we see that $\left\|P_{\gamma}\right\| \geq 1$; in particular, bc $\left\{v_{\gamma}\right\}_{\gamma=0}^{\Gamma} \geq 1$. A long Schauder basis is called normalized if $\left\|v_{\gamma}\right\|=1 \forall 1 \leq \gamma<\Gamma$. It is called monotone if bc $\left\{v_{\gamma}\right\}_{\gamma=0}^{\Gamma}=1$, i.e., its associated projections satisfy $\left\|P_{\gamma}\right\|=1$ for every $1 \leq \gamma<\Gamma$. 
Spaces admitting a fundamental biorthogonal system are characterized as those admitting a quotient of the same density ${ }^{2}$ and having a long Schauder basis. We can see that in the following result, which appears in [Pli80], and whose proof can be found, for example, in [Háj+07].

Theorem 3.1.34 Let $X$ be a Banach space with $\Omega=\operatorname{dens} X>\omega$. The following statements are equivalent:

1. $X$ has a fundamental biorthogonal system of cardinality $\Omega$.

2. $X$ has a quotient with a monotone $\Omega$-long Schauder basis.

3. $X$ has a quotient with a fundamental system of cardinality $\Omega$.

4. $X$ has a $(4+\varepsilon)$ - bounded fundamental biorthogonal system of cardinality $\Omega$.

Now, we will enunciate one more result before showing the main result of this section. It is known as the Godun lifting theorem and is used to lift the biorthogonal system of the space.

Lemma 3.1.35 Let $\left\{z_{\alpha}^{n}, \psi_{\alpha}^{n}\right\}_{\alpha<\Omega, n \in \mathbb{N}}$ be a C-bounded fundamental biorthogonal system in the quotient $X / Y$ such that $\psi_{\alpha}^{n} \in Y^{\perp} \hookrightarrow X^{*}$ has the following properties:

1. $\left\|z_{\alpha}^{n}\right\| \leq C,\left\|\psi_{i}^{n}\right\|=1$.

2. dens $X=\Omega$.

3. For every $\alpha<\Omega$, the sequence $\left\{z_{\alpha}^{n}\right\}_{n=1}^{\infty}$ is not equivalent to the unit basis of $\ell_{1}$.

Let $J: X \rightarrow X / Y$ be the canonical quotient mapping. Then, for every $\varepsilon>0$, the system $\left\{\left(\hat{x}_{\alpha}^{n}, \psi_{\alpha}^{n}\right)\right\}_{\alpha<\Omega, n \in \mathbb{N}}$ admits a lifting to a fundamental biorthogonal system $\left\{\left(x_{\alpha}^{n}, \psi_{\alpha}^{n}\right)\right\}_{\alpha<\Omega, n \in \mathbb{N}}$, with $J\left(x_{\alpha}^{n}\right)=\hat{x}_{\alpha}^{n}$ in $X$ such that

$$
\left\|x_{\alpha}^{n}\right\| \leq 2 C+\varepsilon
$$

We have now the tools for enunciating and demonstrating the following result:

\section{Theorem 3.1.36-GODUN AND TROYANSKI.}

If $X$ has a biorthogonal system with cardinality equal to dens $X$, then for each $\varepsilon \in(0,1), X$ admits an equivalent norm $|\cdot|$ such that $(X,|\cdot|)$ has property $(\alpha, \varepsilon)$.

Proof: Let $Z=\overline{\operatorname{span}}\left\{x_{i}\right\}_{i \in I}$, where $\left\{x_{i}\right\}_{i \in I}$ is the subset of $X$ in the biorthogonal system. Therefore, $Z$ has a quotient space $E=Z / Y$ such that dens $E=\operatorname{dens} Z$, and $E$ has a separable projectional decomposition, as we have seen in Theorem 3.1.34. Then, there exists a transfinite set of projections $P_{\alpha}: E \rightarrow E, \alpha<\alpha_{0}$, such that

1. $\left\|P_{\alpha}\right\|=1$.

2. $P_{\alpha} P_{\beta}=P_{\min (\alpha, \beta)}$.

3. The space $E_{\alpha}=Q_{\alpha} E$ is infinite-dimensional and separable for every $\alpha<\alpha_{0}$, where $Q_{\alpha}=P_{\alpha+1}-P_{\alpha}$.

4. $\overline{\operatorname{span}}\left(\bigcup_{\alpha<\alpha_{0}} E_{\alpha}\right)=E$.

We can get that, for any $\eta>0$, there is a $(1+\eta)$-bounded fundamental biorthogonal system $\left\{\left(e_{\alpha, n}, e_{\alpha, n}^{*}\right)\right\}_{n \in \mathbb{N}}$ such that the system $\left\{e_{\alpha, n}\right\}_{n \in \mathbb{N}}$ is not equivalent to the unit vector basis of $\ell_{1}$.

\footnotetext{
${ }^{2}$ The density of a topological space $T$ is the smallest cardinal such that $T$ has a dense subset of cardinality
} 
Now, let $f_{\alpha, n}=Q_{\alpha}^{*} e_{\alpha, n}^{*}$. Then, $\left\{\left(e_{\alpha, n}, f_{\alpha, n}\right)\right\}_{\alpha<\alpha_{0}, n \in \mathbb{N}}$ is a $(2+2 \eta)$-bounded fundamental biorthogonal system in $E$.

Let $T: X \rightarrow E$ be the quotient map. According to Godun's lifting theorem (Theorem 3.1.35), there exists a system $\left\{u_{\alpha, n}\right\}$ in $Y$ such that $T u_{\alpha, n}=e_{\alpha, n}$, and verifying that $u_{\alpha, n}$ is not equivalent to the unit vector basis of $\ell_{1}$ for every $\alpha<\alpha_{0}$ and $\left\{\left(u_{\alpha, n}, T^{*} f_{\alpha, n}\right)\right\}_{\alpha<\alpha_{0}, n \in \mathbb{N}}$ is a $(4+5 \eta)$-bounded fundamental biorthogonal system on $Y$.

Using the Hahn-Banach theorem, we can find norm-preserving extensions of the dual functionals, so we can relabel this as the biorthogonal system $\left\{\left(y_{i, n}, y_{i, n}^{*}\right)\right\}_{(i, n) \in I \times \mathbb{N}}$, where $|I|=\operatorname{dens} X$ such that

$$
\left\|y_{i, n}\right\|=1,\left\|y_{i, n}^{*}\right\| \leq c,(i, n) \in I \times \mathbb{N},
$$

where $c=4+5 \eta$ and $\forall i \in I\left\{y_{i, n}\right\}_{n \in \mathbb{N}}$ is not equivalent to the usual basis of $\ell_{1}$. Now, assigning to $y_{i, n}$ a proper sign, we can assume that, for any $i \in I$, there exists a sequence of numbers $\left\{c_{k, i, n}\right\}$ such that

$$
c_{k, i, n} \geq 0, \sum_{n} c_{k, i, n}=1, \lim _{k \rightarrow \infty}\left\|\sum_{n} c_{k, i, n} y_{i, n}\right\|=0 .
$$

Define now $\varepsilon>0$ and $\delta=\varepsilon / c(1+\varepsilon)$ and take $\left\{z_{i}\right\}_{i \in I}$ a dense subset of $\mathbb{B}_{X}$. Denote $x_{i, n}=\delta z_{i}+y_{i, n},(i, n) \in I \times \mathbb{N}$ and

$$
V=\overline{\operatorname{conv}}\left(\left\{ \pm x_{i, n}\right\}_{(i, n) \in I \times \mathbb{N}}\right) .
$$

Clearly, $V \subset(1+\delta) \mathbb{B}_{X}$, and as $\lim _{k \rightarrow \infty}\left\|\sum_{n} c_{k, i, n} y_{i, n}\right\|=0, \delta \mathbb{B}_{X} \subset V$, so that the Minkowski functional of $V$ is an equivalent norm $|\cdot|$ on $X$.

Note that

$$
y_{i, n}^{*}\left(x_{i, n}\right) \geq 1-c \delta>0,(i, n) \in I \times \mathbb{N} .
$$

Therefore, the functionals $x_{i, n}^{*}=y_{i, n}^{*} /\left(y_{i, n}^{*}\left(x_{i, n}\right)\right),(i, n) \in I \times \mathbb{N}$ are well defined and

$$
x_{i, n}^{*}\left(x_{i, n}\right)=1,(i, n) \in I \times \mathbb{N} .
$$

And if $(i, n) \neq(j, m)$ then

$$
\left|x_{i, n}^{*}\left(x_{j, m}\right)\right|=\left|y_{i, n}^{*}\left(\delta z_{j}+y_{j, m}\right)\right| /\left(y_{i, n}^{*}\left(x_{i, n}\right)\right) \leq \varepsilon .
$$

As we have defined $x_{i, n}$, we have $\left|x_{i, n}\right| \leq 1$ for all $(i, n) \in I \times \mathbb{N}$, so it is clear that $\left|x_{i, n}^{*}\right| \geq 1$ for all $(i, n) \in I \times \mathbb{N}$. However, as $V$ is the closed convex hull of $\left\{ \pm x_{i, n}\right\}_{(i, n) \in I \times \mathbb{N}}$, we have that $|f|=\sup _{i, n}\left|f\left(x_{i, n}\right)\right|$ for every $f \in X^{*}$, what implies that $\left|x_{i, n}^{*}\right| \leq 1$. Then, $\left|x_{i, n}\right|=\left|x_{i, n}^{*}\right|=1$, so we conclude that $(X,|\cdot|)$ has property $(\alpha, \varepsilon)$.

In this section, we have improved the result of Schachermayer. However, even though we have seen that every Banach space can be equivalently renormed to verify property $\beta$ (Theorem 3.1.20), we do not have the analogous for property $\alpha$ yet, so a good question is whether we could renorm every Banach space equivalently to satisfy property $\alpha$. The answer to this question is negative, as S. Shelah proved in [She85] the existence of a Banach space 
which is not isomorphic to any space with property $\alpha$, using an axiom for the set theory which is additional to the usual ones. This is the first example of this kind that appeared. Later, admiting the continuum hypothesis, K. Kunen [Neg84] constructed a Hausdorff compact topological space $K$ such that $C(K)$ cannot be renormed equivalently with property $\alpha$.

However, this does not mean that there exists a Banach space which cannot be renormed equivalently to satisfy property $A$. That problem is still open.

\subsubsection{PROPERTIES QUASI- $\alpha$ AND QUASI- $\beta$}

In the previous sections, we have studied the sufficient condition Lindenstrauss gave for property $B$, which was later called property $\beta$. We have already seen some examples of spaces verifying property $\beta$ and some results concerning this property, like the fact that property $\beta$ implies property $B$. In the next sections, we will see more results for property $\beta$.

However, one can prove that property $B$ is stable under $c_{0}$-sums ${ }^{3}$, but a $c_{0}$-sum of spaces with property $\beta$ fails to satisfy $\beta$ unless the constants $\rho$ in the definition of $\beta$ can be taken bounded away from 1 . Thus, it is natural to look for a property weaker than $\beta$ that still implies property $B$ and is stable under $c_{0}$-sums. This property should also be satisfied by some spaces which are not isometric to $c_{0}$-sums of spaces with $\beta$. This is what M.D. Acosta, F.J. Aguirre and R. Payá did in 1996 and published in [AAP96]. The new property is called quasi- $\beta$.

The method they used to find the new property was the following one. By using the HahnBanach theorem and the "reversed" Krein Milman theorem, which appears for example in [Hol75], we can see that the third condition in the definition of property $\beta$ holds if and only if every extreme point of the unit ball $B_{Y^{*}}$ lies in the $\omega^{*}$-closure of the set $\left\{t y_{\lambda}^{*}: \lambda \in \Lambda,|t|=1\right\}$. Thus, we can get a weaker property if we only require a local version of the second condition of the definition of $\beta$. More specifically, the estimate in the second condition of $\beta$ is only required for those functionals $y_{\lambda}^{*}$ which are actually needed to approximate each extreme point in $B_{Y}^{*}$.

The definition of property quasi- $\beta$ is the following one.

Definition 3.1.37 - PROPERTY QUASI- $\beta$.

We say that a Banach space $Y$ has property quasi- $\beta$ if there exist a subset $A \subset \mathbb{S}_{Y^{*}}$, a mapping $\sigma: A \rightarrow \mathbb{S}_{Y}$ and a real-valued function $\rho$ on $A$ satisfying the following conditions:

1) $y^{*}\left(\sigma\left(y^{*}\right)\right)=1 \quad \forall y^{*} \in A$.

2) $\left|z^{*}\left(\sigma\left(y^{*}\right)\right)\right| \leq \rho\left(y^{*}\right)<1 \quad \forall y^{*}, z^{*} \in A, y^{*} \neq z^{*}$.

3) For every extreme point $e^{*}$ in the unit ball of $Y^{*}$, there is a subset $A_{e^{*}}$ of $A$ and a scalar $t$ with $|t|=1$ such that $t e^{*}$ lies in the $\omega^{*}$-closure of $A_{e^{*}}$ and $\sup \left\{\rho\left(y^{*}\right): y^{*} \in A_{e^{*}}\right\}<1$.

Notation 3.3. In case $Y$ satisfies property quasi- $\beta$, if it is necessary to be more precise, we sometimes say that $Y$ satisfies property quasi- $\beta(A, \sigma, \rho)$.

Now we show the relation between properties $\beta$ and quasi- $\beta$. If $Y$ has property $\beta$ for sets $\left\{y_{\lambda}: \lambda \in \Lambda\right\} \subset Y$ and $\left\{y_{\lambda}^{*}: \lambda \in \Lambda\right\} \subset Y^{*}$ and a certain constant $0 \leq \rho<1$, then we already know that every extreme point of $\mathbb{B}_{Y^{*}}$ lies in the $\omega^{*}$-closure of the set $\left\{t y_{\lambda}^{*}: \lambda \in \Lambda,|t|=1\right\}$, so $Y$ has property quasi- $\beta(A, \sigma, \rho)$ where $A=\left\{y_{\lambda}^{*}: \lambda \in \Lambda\right\}, \sigma\left(y_{\lambda}^{*}\right)=y_{\lambda}$ for $\lambda \in \Lambda$ and $\rho$ is constant.

\footnotetext{
${ }^{3}$ Given a family of Banach spaces $\left\{Y_{\lambda}: \lambda \in \Lambda\right\}$, we define their $c_{0}$-sum, denoted by $\left(\oplus_{\lambda \in \Lambda} Y_{\lambda}\right)_{c_{0}}$, as the subspace of the cartesian product $\Pi_{\lambda \in \Lambda} Y_{\lambda}$ formed by the families $\left(y_{\lambda}\right)_{\lambda \in \Lambda}$ such that, for every $\varepsilon>0$, the set $\left\{\lambda \in \Lambda:\left\|y_{\lambda}\right\| \geq \varepsilon\right\}$ is finite.
} 
Once the previous property has been defined, we can state the main result that appears in [AAP96].

Theorem 3.1.38 Property quasi- $\beta$ implies property $B$.

In the proof of this theorem, they use the Bishop-Phelps theorem, the Zizler theorem and a lemma that is consequence of the Representation Theorem of Choquet-Bishop-De Leeuw, which proves that if an adjoint operator attains its norm, then it happens at an extreme point.

R It is clear from the previous theorem that if $X$ has property quasi- $\beta$, then $X$ has property $B$, and, in particular, $\overline{N A(X)}=L(X)$.

In the same paper, they discuss the stability of property quasi- $\beta$, for what they prove the two following propositions.

Proposition 3.1.39 Property $B$ is stable under $c_{0}$-sums.

Proof: Let $\Lambda$ be an arbitrary non-empty set and consider $Y=\left(\bigoplus_{\lambda \in \Lambda} Y_{\lambda}\right)_{c_{0}}$, where the Banach space $Y_{\lambda}$ has property $B \quad \forall \lambda \in \Lambda$.

For $T \in L(X, Y)$ with $\|T\|=1$, let us write $T_{\lambda}=P_{\lambda} T$, where $P_{\lambda}$ is the natural projection of $Y$ onto $Y_{\lambda}$ for each $\lambda$. Given $\varepsilon>0$, let $\alpha \in \Lambda$ be such that $\left\|T_{\alpha}\right\|>1-\varepsilon / 2$. Then, as $Y_{\alpha}$ has property $B$, there exists $S_{\alpha} \in N A\left(X, Y_{\alpha}\right)$ such that $\left\|S_{\alpha}\right\|=1$ and $\left\|S_{\alpha}-T_{\alpha}\right\|<\varepsilon$.

Now, we consider $S \in L(X, Y)$ such that $P_{\lambda} S=T_{\lambda}$ for $\lambda \neq \alpha$ and $P_{\alpha} S=S_{\alpha}$. Then, it is clear that $\|S-T\|<\varepsilon$ and $S \in N A(X, Y)$.

Proposition 3.1.40 Property quasi- $\beta$ is stable under $c_{0}$-sums.

Proof: With the same notation as in the proof of the previous proposition, we assume that each Banach space $Y_{\lambda}$ actually satisfies property quasi- $\beta\left(A_{\lambda}, \sigma_{\lambda}, \rho_{\lambda}\right)$ for each $\lambda \in \Lambda$.

Let $I_{\lambda}$ be the natural embedding of $Y_{\lambda}$ into $Y$. We consider the standard identification $Y^{*}=\left(\bigoplus_{\lambda \in \Lambda} Y_{\lambda}^{*}\right)_{\ell_{1}}$. Thus, $P_{\lambda}^{*}$ is the natural embedding of $Y_{\lambda}^{*}$ into $Y^{*}$. An extreme point of $\mathbb{B}_{Y^{*}}$ must be of the form $P_{\lambda}^{*} e_{\lambda}^{*}$, with $e_{\lambda}^{*} \in \mathbb{B}_{Y_{\lambda}^{*}}$ an extreme point and $\lambda \in \Lambda$.

Then, it is easy to verify that $Y$ has property quasi- $\beta(A, \sigma, \rho)$, with $A=\bigcup_{\lambda \in \Lambda} P_{\lambda}^{*} A_{\lambda}$ and

$$
\sigma\left(P_{\lambda}^{*} y_{\lambda}^{*}\right)=I_{\lambda}\left(\sigma_{\lambda}\left(y_{\lambda}^{*}\right)\right), \quad \rho\left(P_{\lambda}^{*} y_{\lambda}^{*}\right)=\rho_{\lambda}\left(y_{\lambda}^{*}\right)
$$

for every $y_{\lambda}^{*} \in A_{\lambda}$ and $\lambda \in \Lambda$.

As a consequence of this proposition and Partington renorming theorem, we have the following corollary, which can be found in [Agu95]. 
Corollary 3.1.41 Every Banach space over $\mathbb{R}$ with dimension greater than two can be equivalently renormed so that it verifies property quasi $\beta$ and does not have property $\beta$.

Proof: Let $Y$ be a Banach space of dimension greater than two. We take a subspace of dimension three, which we will call $Y_{0}$ and we renorm it using the same procedure we will use in the following example. Let $Y_{1}$ be a complement of $Y_{0}$ and, using the Partington theorem, renorm $Y_{1}$ with property $\beta$.

If we define now

$$
\left\|y_{0}+y_{1}\right\|=\max \left\{\left\|y_{0}\right\|_{0},\left\|y_{1}\right\|_{1}\right\} \quad\left(y_{0} \in Y_{0}, y_{1} \in Y_{1}\right)
$$

where $\|\cdot\|_{0}$ and $\|\cdot\|_{1}$ are, respectively, the norms defined in $Y_{0}$ and $Y_{1}$, then we obtain a new norm in $Y$ which has property quasi- $\beta$ (for the previous proposition) but does not verify property $\beta$.

We will present now an example of a finite dimensional space with property quasi- $\beta$ which is not isometric to a $c_{0}$-sum of spaces with property $\beta$. This example has also been taken from [AAP96].

- Example 3.1.42 Let us consider $a_{n}=\left(\sin \pi / 2^{n}, \cos \pi / 2^{n}, 0\right)$ and the set

$$
A=\left\{a_{n}: n \in \mathbb{N}\right\} \cup\{(0,1,1),(0,1,-1)\} \subset \mathbb{R}^{3} .
$$

Take $Y$ as $\mathbb{R}^{3}$ with the norm that makes $\mathbb{B}_{Y^{*}}=\operatorname{co}(A \cup-A)$.

The set of extreme points of $\mathbb{B}_{Y^{*}}$ is $A \cup-A$, what makes the third condition in the definition of quasi- $\beta$ trivially satisfied.

Then, $Y$ has property quasi- $\beta(A, \sigma, \rho)$, with

$$
\begin{gathered}
\sigma\left(a_{n}\right)=a_{n} \quad \forall n \in \mathbb{N}, \\
\sigma(0,1, \pm 1)=(0,1 / 2, \pm 1 / 2), \\
\rho\left(a_{n}\right)=\cos \pi / 2^{n+1} \quad \forall n \in \mathbb{N}, \\
\rho(0,1, \pm 1)=1 / 2 .
\end{gathered}
$$

However, we have previously seen that a finite-dimensional space has property $\beta$ if, and only if, its unit ball is a polyhedron. As the unit ball of $Y$ is not a polyhedron, $Y$ fails property $\beta$.

The stability by $\ell_{1}$-sums is more delicated. We can find in [Agu95] that properties $\beta$ and quasi- $\beta$ are stable by finite $\ell_{1}$-sums of real spaces.

We have shown some properties and examples of spaces with property $\beta$. In the following sections, we will see more examples and properties, but, first, we need to introduce some more concepts.

Some years after the definition of property quasi- $\beta$, the authors Y.S. Choi and H. G. Song defined the analogous property quasi- $\alpha$ in [CS08], whose dual property is property quasi- $\beta$. This property implies property $A$, as property quasi- $\beta$ implied property $B$. We are going to show, like they do in their paper, how to obtain property quasi- $\alpha$ from the properties we have defined before.

Firstly, let us remember the third condition in the definition of property $\beta$ : 


$$
\|y\|=\operatorname{Sup}\left\{\left|y_{\lambda}^{*}(y)\right|: \lambda \in \Lambda\right\}, \quad \forall y \in Y .
$$

We call $A=\left\{x_{\lambda}: \lambda \in \Lambda\right\}$ and $A^{*}=\left\{x_{\lambda}^{*}: \lambda \in \Lambda\right\}$. By the third condition, $A^{*}$ is a norming $\operatorname{set}^{4}$ for $X$. Since the $\omega^{*}$-closed absolute convex hull of $A^{*}$ is the unit ball of $X^{*}$ (by the separation theorem), the previous condition can be replaced by:

$$
\mathbb{B}_{Y^{*}}={\overline{\Gamma\left(A^{*}\right)}}^{\omega^{*}},
$$

where $\Gamma\left(A^{*}\right)$ is the absolute convex hull of $A^{*}$.

Now, if we remember property $\alpha$, the third condition of the definition was: The absolute convex hull of the set $\left\{x_{\lambda}: \lambda \in \Lambda\right\}$ is dense in the unit ball of $X$, i.e.,

$$
\overline{\Gamma(A)}=\mathbb{B}_{X} \text {. }
$$

Thus, we can see a duality between properties $\alpha$ and $\beta$. We want to extend this duality to a duality between properties quasi- $\alpha$ and quasi- $\beta$. For that, let us have a look at the definition of property quasi- $\beta$. To make it look more like properties $\alpha$ and $\beta$, we can rewrite that definition as follows.

We say that a Banach space $X$ has property quasi- $\beta$ if, for some index set $\Lambda$, there exist $A=\left\{x_{\lambda} \in \mathbb{S}_{X}: \lambda \in \Lambda\right\}, A^{*}=\left\{x_{\lambda}^{*} \in \mathbb{S}_{X^{*}}: \lambda \in \Lambda\right\}$ and $\rho: A^{*} \rightarrow \mathbb{R}$ such that

1) $x_{\lambda}^{*}\left(x_{\lambda}\right)=1 \quad x_{\lambda}^{*} \in A^{*}, x_{\lambda} \in A$.

2) $\left|x_{\alpha}^{*}\left(x_{\lambda}\right)\right| \leq \rho\left(x_{\lambda}^{*}\right)<1 \quad x_{\alpha}^{*}, x_{\lambda}^{*} \in A^{*}, x_{\lambda} \in A, \alpha \neq \lambda$.

3) For every extreme point $e^{*}$ in the unit ball of $X^{*}$, there is a subset $A_{e^{*}}$ of $A$ and a scalar $t$ with $|t|=1$ such that $t e^{*} \in \overline{A_{e^{*}} \omega^{*}}$ and $r_{e^{*}}=\sup \left\{\rho\left(x^{*}\right): x^{*} \in A_{e^{*}}\right\}<1$.

Now, from this, we can define property quasi- $\alpha$ :

\section{Definition 3.1.43 - PROPERTY QUASI- $\alpha$.}

We say that a Banach space $X$ has property quasi- $\alpha$ if, for some index set $\Lambda$, there exist $A=\left\{x_{\lambda} \in \mathbb{S}_{X}: \lambda \in \Lambda\right\}, A^{*}=\left\{x_{\lambda}^{*} \in \mathbb{S}_{X^{*}}: \lambda \in \Lambda\right\}$ and $\rho: A \rightarrow \mathbb{R}$ such that:

1) $x_{\lambda}^{*}\left(x_{\lambda}\right)=1 \quad x_{\lambda}^{*} \in A^{*}, x_{\lambda} \in A$.

2) $\left|x_{\lambda}^{*}\left(x_{\alpha}\right)\right| \leq \rho\left(x_{\lambda}\right)<1 \quad x_{\lambda}^{*} \in A^{*}, x_{\alpha}, x_{\lambda} \in A, \alpha \neq \lambda$.

3) For every extreme point $e$ in the unit ball of $X^{* *}$, there is a subset $A_{e}$ of $A$ and a scalar $t$ with $|t|=1$ such that $t e \in{\overline{J\left(A_{e}\right)}}^{\omega^{*}}$ and $r_{e}=\sup \left\{\rho(x): x \in A_{e}\right\}<1$, where $J: X \hookrightarrow X^{* *}$ is the canonical embedding .

With this definition, we can see that $\overline{\Gamma(A)}=\mathbb{B}_{X}$ using the separation theorem and the fact that the set of extreme points of $\mathbb{B}_{X^{* *}}$ is contained in the $\omega^{*}$-closed absolute convex hull of $J(A)$, hence $\mathbb{B}_{X^{* *}}=\overline{\Gamma(J(A))}^{\omega^{*}}$. Then, given $T \in L(X, Y)$, we have

$$
\|T\|=\sup _{x_{\lambda} \in A}\left\|T\left(x_{\lambda}\right)\right\| \text {. }
$$

As we did for property quasi- $\beta$, we now show the relation between property $\alpha$ and property quasi- $\alpha$. Let us consider a Banach space $X$ with property $\alpha$ and the set $A$ in the definition of that property. Then, it is easy to see that $X$ has property quasi- $\alpha$, by taking $A_{e}=A$ for every $e \in \operatorname{Ext}\left(\mathbb{B}_{X^{* *}}\right)$ and obtaining that $\rho$ is constant.

We can also find the following result in [CS08], analogous to the one with property quasi- $\beta$.

\footnotetext{
${ }^{4}$ We say that $Y$ is a norming set for $X$ if the mapping $T: X \rightarrow T(X)$ defined by $T(x)=\{y(x)\}_{y \in Y}$ is injective.
} 
Theorem 3.1.44 Property quasi- $\alpha$ implies property $A$.

R We can deduce from this theorem that if $X$ has property quasi- $\alpha$, then $X$ has property $A$, and, in particular, $\overline{N A(X)}=L(X)$.

In proposition 3.1.23, we showed a result of Schachermayer relating the property $\alpha$ of a Banach space with the property $\beta$ of its dual. Now, we consider the duality between properties quasi- $\alpha$ and quasi- $\beta$. For that, we first give the following definition.

Definition 3.1.45 Let $Y$ be a Banach space and $X$ a subspace of $Y^{*}$. We say that $Y$ has property quasi- $\beta$ induced by $X$ if $Y$ has property quasi- $\beta$ and $A^{*} \subset X$ for the set $A^{*}$ in the definition of property quasi- $\beta$.

Now we can enunciate the theorem of duality.

Theorem 3.1.46 Let $X$ be a Banach space and $Y=X^{*}$. Then,

$X$ has property quasi- $\alpha \Leftrightarrow Y$ has property quasi- $\beta$ induced by $X$

In particular, if $Z$ is a reflexive Banach space, then $Z$ has property quasi- $\alpha$ (resp. quasi- $\beta$ ) if and only if $Z^{*}$ has property quasi- $\beta$ (resp. quasi- $\alpha$ ).

Proof: Let $A, A^{*}$ and $\rho$ be the sets and the function in the definition of property quasi- $\alpha$ of $X$. For $Y=X^{*}$, we take $A_{Y}=A^{*}$ and $A_{Y}^{*}=J(A)$, where $J: X \hookrightarrow X^{* *}$ is the canonical embedding.

Then, if we define $\rho_{Y}: A_{Y}^{*} \rightarrow \mathbb{R}$ by $\rho\left(J\left(x_{\lambda}\right)\right)=\rho\left(x_{\lambda}\right)$, we can see that the proof follows immediately.

When we studied property quasi- $\beta$, we saw that property $B$ is stable under $c_{0}$-sums while property $\beta$ is not. Then, property quasi- $\beta$ was defined so that it may be stable under $c_{0}$-sums.

Analogously, we can show that property $A$ is stable under $\ell_{1}$-sums while property $\alpha$ is not.

Theorem 3.1.47 Property $A$ is stable under $\ell_{1}$-sums.

Let $X_{\lambda}$ have property $A$ for every $\lambda \in \Lambda$, where $\Lambda$ is an index set. Then, $X=\left(\bigoplus_{\lambda \in \Lambda} X_{\lambda}\right)_{\ell_{1}}$ also has property $A$.

Proof: $\quad$ Let $Y$ be a Banach space and $T \in L(X, Y)$ with $\|T\|=1$. We write $T=\bigoplus_{\lambda \in \Lambda} T_{\lambda}$, with $T_{\lambda} \in L\left(X_{\lambda}, Y\right)$. Given $0<\varepsilon<1$, since $\|T\|=\sup \left\|T_{\lambda}\right\|$, there exists $\alpha \in \Lambda$ such that

$$
\left\|T_{\alpha}\right\|>1-\varepsilon .
$$

As $X_{\alpha}$ has property $A$, there exists $S_{\alpha} \in N A\left(X_{\alpha}, Y\right)$ such that $\left\|T_{\alpha}-S_{\alpha}\right\|<\varepsilon$ and $\left\|S_{\alpha}\right\|>$ $1-\varepsilon$.

We consider 


$$
S=\bigoplus_{\lambda \in \Lambda} S_{\lambda}
$$

where $S_{\lambda}=(1-\varepsilon) T_{\lambda}$ for $\lambda \neq \alpha$. Then, $S$ attains its norm and $\|T-S\|<\varepsilon$

We have just seen that property $A$ is stable under $\ell_{1}$-sums. Now, we will show that property $\alpha$ and property quasi- $\alpha$ are not stable under $\ell_{1}$-sums. For that, it is enough to look at the following example.

- Example 3.1.48 For every positive integer $n \geq 2$, let $X_{n}$ be the Banach space $\mathbb{R}^{2}$ with the unit ball

$$
B_{n}=\operatorname{co}\left(A^{n} \cup-A^{n}\right),
$$

where

$$
A^{n}=\left\{p_{k}=\left(\cos \left(\frac{k \pi}{n}\right), \sin \left(\frac{k \pi}{n}\right)\right) \in \mathbb{R}^{2}: 0 \leq k \leq n-1\right\} .
$$

Then, we can see that each space $X_{n}$ has property $\alpha$ with the set $A^{n}$ and the constant $\rho_{n}=\cos \left(\frac{\pi}{n}\right)$ (hence, every $X_{n}$ has property quasi- $\alpha$ ). However, if we consider

$$
X=\left(\bigoplus_{n \geq 2} X_{n}\right)_{\ell_{1}},
$$

we can verify that $X$ does not have property quasi- $\alpha$ (hence, $X$ does not have property $\alpha$ ). The proof can also be found in [CS08].

We have found an example to show that property quasi- $\alpha$ is not stable under $\ell_{1}$-sums. However, in the same paper, they also proved the following proposition.

Proposition 3.1.49 Property quasi- $\alpha$ is stable under finite $\ell_{1}$-sums.

We will show more properties and examples of spaces with property $\alpha$ or property quasi- $\alpha$ in the following sections.

\subsection{Bishop-PhelPS AND RADON-NIKOdYM PROPERTIES}

In the previous sections, we have changed the chronological order of the development of the theory of norm-attaining operators. We have already mentioned two really important papers in the beginning of this theory, the ones of Lindenstrauss (1963) and Zizler (1973). The following significant advance appeared in 1977, when J. Bourgain published in [Bou97] what can be considered the most brilliant results in this theory. The most significant advance was finding the relation between the problem of density of norm-attaining operators and the Radon-Nikodym property (RNP).

In this section, we are working with one specific formulation of the RNP. An important reference for the RNP is the book of Diestel and Uhl, [DU77]. In this book, approximately thirty equivalent formulations for the RNP appear, and the one that Bourgain demonstrated, and we are going to show here, corresponds to number 14. 
Before studying this equivalent formulation for the RNP, let us remember the Radon-Nikodym theorem and the Radon-Nikodym property.

\section{Theorem 3.2.1 - RADON-NIKODYM THEOREM.}

Let $(\Omega, \mathscr{A}, \mu)$ be a measurable space with $\mu$ a $\sigma$-finite measure and let $\lambda$ be a complex measure defined in the $\sigma$-algebra $\mathscr{A}$ that is absolutely continuous respect to $\mu$. Then, there exists a function $f \in L^{1}(\mu)$ such that

$$
\lambda(E)=\int_{E} f d \mu \quad(E \in \mathscr{A}) .
$$

Moreover, if $\lambda$ is real, we can make $f(\Omega) \subseteq \mathbb{R}$, and, if $\lambda$ is positive, $f(\Omega) \subseteq[0, \infty[$.

The Radon-Nikodym property has its origin in trying to obtain versions of the RadonNikodym theorem for vectorial measures.

\section{Definition 3.2.2 - RADON-NIKODYM PROPERTY.}

A Banach space $X$ has the Radon-Nikodym property with respect to $(\Omega, \Sigma, \mu)$ if for each $\mu$-continuous vector measure $G: \Sigma \rightarrow X$ of bounded variation there exists $g \in L^{1}(\mu, X)$ such that $G(E)=\int_{E} g d \mu$ for all $E \in \Sigma$.

A Banach space $X$ has the Radon-Nikodym property if $X$ has the Radon-Nikodym property with respect to every finite measure space.

In 1967, M. Rieffel began the treating of the RNP as a geometric property of Banach spaces. In his work, he introduced the notion of dentability and began studying its relation with the RNP.

\section{Definition 3.2.3 - DENTABILITY.}

Let $C$ be a subset of a Banach space $X$. We say that $C$ is dentable if, for every $\varepsilon>0$, we can find a point $x \in C$ such that $x$ does not belong to the closed convex hull of the set obtained by removing from $C$ the ball with center $x$ and radio $\varepsilon$ :

$$
x \notin \overline{\operatorname{co}}\left(C \backslash\left(x+\varepsilon \mathbb{B}_{X}\right)\right) .
$$

As a result of the work of Rieffel, Maynard, Huff, Davis and Phelps, they obtained that a Banach space $X$ has the RNP if, and only if, every bounded subset of $X$ is dentable, the characterization of the RNP we are using in the rest of the chapter. Now, we will use the following characterization of non-dentable sets, obtained by Huff and Morris (see [DU77], Theorem VII.4.1.).

Proposition 3.2.4 Let $C$ be a non-empty, convex, closed, bounded subset of a Banach space $X$. Then, $C$ is non-dentable if, and only if, there exists $\varepsilon>0$ such that, for every $F$ finite subset of $X$, we have $C=\overline{\mathrm{co}}\left(C \backslash F+\varepsilon \mathbb{B}_{X}\right)$.

Before beginning with the first results of the work of Bourgain, we need the following definition, as a generalization of property A. 


\section{Definition 3.2.5 - BISHOP-PHELPS PROPERTY.}

Let $C$ be a convex, closed and bounded subset of a Banach space $X$. We say that $C$ has Bishop-Phelps property if, for every Banach space $Y$, the set of the operators $T \in L(X, Y)$ such that the function $x \mapsto\|T x\|$ attains its maximum in $C$ is dense in $L(X, Y)$ for the topology of the norm.

R It is evident that a Banach space $X$ has property $A$ if, and only if, its unit ball $\mathbb{B}_{X}$ has Bishop-Phelps property.

We say that a Banach space $X$ has Bishop-Phelps property (BPp) if every convex, closed and bounded subset of $X$ has Bishop-Phelps property.

Then, for our purpose of studying Banach spaces verifying $\overline{N A(X)}=L(X)$, the following result is clear.

Result 3.2.6 If $X$ is a Banach space with the BPp, then

$$
\overline{N A(X)}=L(X) \text {. }
$$

Now, we can enunciate the first result in [Bou97], the work of Bourgain (we are using the nomenclature that appears in [DU77]).

\section{Theorem 3.2.7 - BOURGAIN THEOREM.}

Let $C$ be a convex, closed and bounded subset of a Banach space $X$ and suppose that $C$ is separable. Then, if $C$ has the Bishop-Phelps property, $C$ is dentable.

The proof of this result is inspired in the arguments used by Lindenstrauss to construct the first counterexamples for the problem of density of the set of norm-attaining operators.

Proof: Let us suppose that $C$ is not dentable. Then, we will prove that $C$ cannot have the BPp.

Applying Proposition 3.2.4, there exists $\varepsilon>0$ such that

$$
C=\overline{\mathrm{co}}\left[C \backslash\left(F+\varepsilon \mathbb{B}_{X}\right)\right]
$$

for every finite $F \subseteq X$. We have the hypothesis that $C$ is separable; then, $Z$, the closed subspace of $X$ generated by $C$, is also separable. Let $\left\{z_{n}: n \in \mathbb{N}\right\}$ be a countable, dense subset in $Z$.

For every $n \in \mathbb{N}$, denote by $Z_{n}$ the unidimensional subspace of $Z$ generated by $z_{n}$. Consider the application $\Phi: X \rightarrow \ell_{2}$ defined by

$$
\begin{gathered}
{[\Phi(x)](0)=\|x\|,} \\
{[\Phi(x)](n)=2^{\frac{n}{2}} \operatorname{dist}\left(x, Z_{n}\right) \quad \forall n \in \mathbb{N} .}
\end{gathered}
$$

It is clear that $\Phi$ is not linear. However, it verifies

$$
[\Phi(x+y)](n) \leq[\Phi(x)](n)+[\Phi(y)](n)
$$

for $n \in \mathbb{N} \cup\{0\}, x, y \in X$. Using now the monotonicity of the norm of $\ell_{2}$, if we define 


$$
\|\mid\| x\|=\| \Phi \|_{\ell_{2}} \quad \forall x \in X
$$

we obtain a norm ||$|\cdot|||$ in $X$ equivalent to the norm we began with. In fact, we have

$$
\|x\| \leq\|x\| \mid \leq \sqrt{2}\|x\| \quad \forall x \in X .
$$

The first inequality is clear, as $\|x\|=[\Phi(x)](0) \leq\|\Phi(x)\|_{\ell_{2}}=\|\| x\|\|$, and the second one comes from the definition of $\Phi$. For proving that $C$ does not have the Bishop-Phelps property, take $Y=(X,\||\cdot|\|)$ (it is clear that we are taking $X=(X,\|\cdot\|)$ ) and consider $I: X \rightarrow Y$, the identity operator. We will prove that this operator cannot be approximated by operators which attain their maximum norm in $C$.

Consider $T$ an operator from $X$ to $Y$ such that $\|I-T\|<\frac{\varepsilon}{4}$ and suppose that $T$ attains its maximum norm in $C$. Then, there exists $x_{0} \in C$ such that

$$
\||T x|\| \leq\left\|\left|T x_{0} \|\right| \quad \forall x \in C .\right.
$$

Fix $k \in \mathbb{N}$ such that $\left\|x_{0}-z_{k}\right\|<\frac{\varepsilon}{4}$ (it is clear that this $k$ exists, as $\left\{z_{n}\right\}$ is a dense subset of $Z$, the closed subspace of $X$ generated by $C$ ). Cover the unit ball centered in zero with radius $1+\varepsilon$ in the space $Z_{k}$ by a finite number of balls of radius $\frac{\varepsilon}{8}$. Let $F$ be the finite set of the centers of these balls.

If we take $y \in C$ and $\operatorname{dist}\left(y, Z_{k}\right)<\frac{7 \varepsilon}{8}$, and $z \in Z_{k}$ verifying $\|y-z\|<\frac{7 \varepsilon}{8}$, we have $\|z\| \leq$ $\|y\|+\frac{7 \varepsilon}{8}<1+\varepsilon$. Then, there exists $u \in F$ with $\|z-u\|<\frac{\varepsilon}{8}$, so $\|y-u\| \leq\|y-z\|+\|z-u\|<\varepsilon$.

Summing up all of this, the set

$$
D=\left\{y \in C: \operatorname{dist}\left(y, Z_{k}\right)<\frac{7 \varepsilon}{8}\right\}
$$

verifies $D \subseteq F+\varepsilon \mathbb{B}_{X}$. Hence,

$$
C=\overline{\mathrm{co}}\left(C \backslash\left(F+\varepsilon \mathbb{B}_{X}\right)\right) \subset \overline{\mathrm{co}}(C \backslash D) \subset C .
$$

Therefore,

$$
2\left|\left\|T x_{0}|\||=\operatorname{Max}\left\{\left|\left\|T x_{0}+T x \mid\right\|: x \in C\right\}=\operatorname{Sup}\left\{\left\||| T x_{0}+T x \mid\right\|: x \in C \backslash D\right\},\right.\right.\right.
$$

and there must exist a sequence $\left\{y_{n}\right\} \subset C \backslash D$ so that

$$
|| T x_{0}+T y_{n}|||\rightarrow 2||| T x_{0}|| \mid,
$$

or, equivalently,

$$
\left\|\Phi\left(T x_{0}+T y_{n}\right)\right\|_{\ell_{2}} \rightarrow 2\left\|\Phi\left(T x_{0}\right)\right\|_{\ell_{2}}
$$

Now, as

$$
\left\|\Phi\left(T x_{0}+T y_{n}\right)\right\|_{\ell_{2}} \leq\left\|\Phi\left(T x_{0}\right)+\Phi\left(T y_{n}\right)\right\|_{\ell_{2}} \leq 2\left\|\Phi\left(T x_{0}\right)\right\|_{\ell_{2}},
$$

the Parallelogram Law gives $\left\|\Phi\left(T x_{0}\right)-\Phi\left(T y_{n}\right)\right\|_{\ell_{2}} \rightarrow 0$. In particular, dist $\left(T y_{n}-T x_{0}, Z_{k}\right) \rightarrow 0$; however,

$$
\operatorname{dist}\left(T x_{0}, Z_{k}\right) \leq\left\|x_{0}-T x_{0}\right\|+\operatorname{dist}\left(x_{0}, Z_{k}\right) \leq\|I-T\|+\operatorname{dist}\left(x_{0}, Z_{k}\right) \leq \frac{\varepsilon}{4}+\frac{\varepsilon}{4}=\frac{\varepsilon}{2} .
$$


Thus, for $n$ large enough, we have dist $\left(T y_{n}, Z_{k}\right)<\frac{5 \varepsilon}{8}$. Therefore,

$$
\operatorname{dist}\left(y_{n}, Z_{k}\right) \leq\left\|y_{n}-T y_{n}\right\|+\operatorname{dist}\left(T y_{n}, Z_{k}\right)<\frac{7 \varepsilon}{8},
$$

which is a contradiction with the fact that $y_{n} \in C \backslash D$.

This theorem has the restrictive hypothesis of the set $C$ being separable, which is essential in the demonstration. However, using some other results from the time he published his paper, Bourgain deduced the following consequence of the theorem.

Corollary 3.2.8 Every Banach space with the Bishop-Phelps property has the Radon-Nikodym property.

After the work of Bourgain, and its appearance in the book of Diestel and Uhl, R. Huff [Huf80] improved the result of the previous theorem. For that, he used the following characterization of the RNP, obtained by Davis and Phelps.

Theorem 3.2.9 A Banach space $X$ has the RNP if, and only if, the unit ball of $X$, for every equivalent norm, is dentable.

Then, with this characterization, an easy modification of the demonstration of theorem 3.2.7 leads to the following theorem.

\section{Theorem 3.2.10 - HUFF THEOREM.}

Suppose that a Banach space $X$ does not verify the RNP. Then, there exist norms $\|\cdot\|_{1}$ and $\|\cdot\|_{2}$ in $X$, equivalent to the initial norm, such that, if we denote $X_{1}=\left(X,\|\cdot\|_{1}\right)$ and $X_{2}=\left(X,\|\cdot\|_{2}\right)$, then $N A\left(X_{1}, X_{2}\right)$ is not dense in $L\left(X_{1}, X_{2}\right)$.

We have the two following corollaries as a consequence of this theorem.

Corollary 3.2.11 If $X$ is a Banach space such that every space isomorphic to $X$ has property $A$, then $X$ has the RNP.

Corollary 3.2.12 If $X$ is a Banach space such that every subspace isomorphic to $X$ has property $B$, then $X$ has the RNP.

Before beginning another section, from the Huff theorem we can construct an example of space $X$ such that $\overline{N A(X)} \neq L(X)$. For that, we will prove now a theorem stating that if we have $X$ and $Y$ Banach spaces such that $\overline{N A(X, Y)} \neq L(X, Y)$, then $\overline{N A\left(X \oplus_{\infty} Y\right)} \neq L\left(X \oplus_{\infty} Y\right)$, and the same result holds by intersecting the set of norm-attaining operators with an ideal of $L(X, Y)$, a result extracted from [Mar14], recently published by M. Martín.

We will use this result for the construction of many examples for $\overline{N A(X)} \neq L(X)$ in this and the following chapters. 
Theorem 3.2.13 Let $X$ and $Y$ be Banach spaces such that $\overline{N A(X, Y)} \neq L(X, Y)$. Let us consider $Z=X \oplus_{\infty} Y$. Then, $\overline{N A(Z)} \neq L(Z)$.

Proof: $\quad$ As $\overline{N A(X, Y)} \neq L(X, Y)$, there exists $T \in L(X, Y)$ such that $T \notin \overline{N A(X, Y)}$. Then, we define:

$$
\begin{aligned}
S: Z=X \oplus_{\infty} Y & \rightarrow \quad Z \\
(x, y) & \mapsto T(x)
\end{aligned}
$$

We see that $S(Z) \subseteq Y$. We want to prove that $S \notin \overline{N A(Z)}$.

Suppose, on the contrary, that $S \in \overline{N A(Z)}$. Then, there exist $\left\{S_{n}\right\}_{n \in \mathbb{N}} \subseteq N A(Z)$ such that $\left\{\left\|S-S_{n}\right\|\right\} \rightarrow 0$.

Let $P$ and $Q$ be the projections from $Z$ to $X$ and $Y$, respectively. Then, it is clear that $P S=0$ and $Q S=S$. So $\left\{P S_{n}\right\} \rightarrow 0$ and $\left\{Q S_{n}\right\} \rightarrow S$. And this implies that, for large enough $n$, $\left\|Q S_{n}\right\|>\left\|P S_{n}\right\|$.

For every $S_{n}$, we have

$$
\left\|S_{n}\right\|=\sup _{z \in \mathbb{B}_{Z}}\left\|S_{n}(z)\right\|=\sup _{z \in \mathbb{B}_{Z}}\left\{\max \left\{\left\|P S_{n}(z)\right\|_{X},\left\|Q S_{n}(z)\right\|_{Y}\right\}\right\}=\max \left\{\left\|P S_{n}\right\|,\left\|Q S_{n}\right\|\right\}
$$

Now, if we take $n$ large enough, as $S_{n} \in N A(Z)$ and $\left\|Q S_{n}\right\|>\left\|P S_{n}\right\|$ (then, $\left\|S_{n}\right\|=\left\|Q S_{n}\right\|$ ), there exists $z \in \mathbb{B}_{Z}$ such that

$$
\left\|S_{n}\right\|=\left\|S_{n}(z)\right\|=\max \left\{\left\|P S_{n}(z)\right\|_{X},\left\|Q S_{n}(z)\right\|_{Y}\right\} \leq \max \left\{\left\|P S_{n}\right\|,\left\|Q S_{n}\right\|\right\}=\left\|S_{n}\right\|
$$

Then, $Q S_{n}$ attains its norm, for $n$ large enough. Now, by deleting some terms of the sequence $\left\{S_{n}\right\}$, we can say that $Q S_{n}$ attains its norm $\forall n \in \mathbb{N}$ (and it does at the same points that $S_{n}$ ).

As $S_{n}$ attains its norm $\forall n \in \mathbb{N}$, take $\left(x_{n}, y_{n}\right) \in \mathbb{B}_{Z}$ such that $\left\|S_{n}\left(x_{n}, y_{n}\right)\right\|=\left\|S_{n}\right\|$. Then, take $x_{n}^{*} \in \mathbb{S}_{X^{*}}$ such that $x_{n}^{*}\left(x_{n}\right)=1$. For every $n \in \mathbb{N}$, define $T_{n} \in L(X, Y)$ as

$$
T_{n}(x)=Q S_{n}\left(x, x_{n}^{*}(x) y_{n}\right)
$$

Then, for every $n \in \mathbb{N},\left\|T_{n}\right\| \leq\left\|Q S_{n}\right\|$ and $\left\|T_{n}\left(x_{n}\right)\right\|=\left\|Q S_{n}\left(x_{n}, y_{n}\right)\right\|=\left\|Q S_{n}\right\|=\left\|S_{n}\right\|$, so $T_{n} \in N A(X, Y)$. Moreover, for every $x \in \mathbb{B}_{x}$,

$$
\left\|T_{n}(x)-T(x)\right\|=\left\|Q S_{n}\left(x, x_{n}^{*}(x) y_{n}\right)-Q S\left(x, x_{n}^{*}(x) y_{n}\right)\right\| \leq\left\|Q S_{n}-Q S\right\| \leq\left\|S_{n}-S\right\|
$$

Therefore, as $\left\{\left\|S-S_{n}\right\|\right\} \rightarrow 0, T$ can be approximated by a sequence of norm-attaining operators (contradiction!).

From this theorem and the Huff theorem, we can deduce the following result:

Result 3.2.14 If $Y$ does not verify the RNP, then there exist equivalent norms $\|\cdot\|_{1},\|\cdot\|_{2}$ such that if $Y_{1}=\left(Y,\|\cdot\|_{1}\right), Y_{2}=\left(Y,\|\cdot\|_{2}\right)$ and we take $X=Y_{1} \oplus_{\infty} Y_{2}$, then

$$
\overline{N A(X)} \neq L(X) \text {. }
$$




\subsubsection{THE NON-LINEAR OPTIMIZATION PRINCIPLE OF BOURGAIN AND STEGALL}

Now we are working in the opposite direction. We know that if every equivalent renorming of a Banach space $X$ has property $A$, then $X$ has the RNP; we will now prove that every Banach space with the RNP has property $A$.

In the previous section, the geometric formulation of the RNP in terms of dentability was very important. In this section we will study another important geometric aspect of the RNP, its formulation in terms of the strong exposure.

Definition 3.2.15 - StRONGLY EXPOSED, SLICE.

Let $D$ be a topological Hausdorff space and $\varphi$ a function defined in $D$ with real values. We say that $D$ is strongly exposed by $\varphi$ at a point $x_{0} \in D$ if $\varphi(x) \leq \varphi\left(x_{0}\right)$ for every $x \in D$, and for every sequence $\left\{x_{n}\right\}$ in $D$ such that $\left\{\varphi\left(x_{n}\right)\right\} \rightarrow \varphi\left(x_{0}\right)$, we have $\left\{x_{n}\right\} \rightarrow x_{0}$.

In particular, $\varphi$ has a maximum in $D$ and attains it only in $x_{0}$.

For every bounded function $\varphi: D \rightarrow \mathbb{R}$, we can consider the slices of $D$ determined by $\varphi$; more specifically, given $\delta>0$, we define

$$
S(D, \varphi, \delta)=\{x \in D: \varphi(x)>\operatorname{Sup} \varphi(D)-\delta\} .
$$

In case that $D$ is a complete metric space and $\varphi$ is continuous, it is clear that $D$ is strongly exposed by $\varphi$ if, and only if,

$$
\lim _{\delta \rightarrow 0} \operatorname{diam}(S(D, \varphi, \delta))=0 .
$$

We are now interested in the situation of $D$ being a convex, closed and bounded subset of a Banach space $X$. We will take $X$ real and, for the moment, we will suppose that $\varphi \in X^{*}$.

First, we find a relation between dentability and strongly exposure in the following proposition.

Proposition 3.2.16 Let $X$ be a real Banach space and $D$ a subset of $X$. The following facts are equivalent:

1. $D$ is dentable.

2. For every $\varepsilon>0$, we can find $x^{*} \in X^{*}$ and $\delta>0$ such that diam $\left(S\left(D, X^{*}, \delta\right)\right)<\varepsilon$.

In 1974, R. Phelps proved, in [Phe74], based on a previous result of Lindenstrauss, that if $D$ is a convex, closed and bounded subset of a real Banach space $X$ which verifies the RNP, then the set of the functionals that expose strongly $D$ is dense in $X^{*}$. The work of Bourgain contains an improvement of this result.

Theorem 3.2.17 Let $D$ be a non-empty, convex, closed and bounded subset of a real Banach space $X$. Suppose that every non-empty subset of $D$ is dentable. Then,

$$
\left\{x^{*} \in X^{*}: x^{*} \text { exposes strongly } D\right\}
$$

is a dense set in $X^{*}$ for the topology of the norm.

In 1978, C. Stegall presented in [Ste78] a non-linear version of the previous theorem. Later, in [Ste86], he showed an important simplification of the proof of Bourgain, and also an improvement 
of what he had previously presented. This result is known as the non-linear optimization principle of Bourgain and Stegall.

The new demonstration is mainly based in the relation of duality between Frèchet differentiability ${ }^{5}$ and strong exposure. The non-linear version of the previous theorem is the following one.

Theorem 3.2.18 Let $D$ be a non-empty, convex, closed and bounded subset of a real Banach space $X$. Suppose that every non-empty subset of $D$ is dentable and let $\phi: D \rightarrow \mathbb{R}$ be an upper semicontinuous, bounded function. Then, the set

$$
\left\{x^{*} \in X^{*}: \phi+x^{*} \text { exposes strongly } D\right\}
$$

is dense in $X^{*}$ for the topology of the norm.

The proof of this theorem, apart from the demonstration of the result of Bourgain, uses the following interesting lemma.

Lemma 3.2.19 Let $X$ and $Y$ be real Banach spaces and $D, E$ convex, closed, bounded subsets of $X$ and $Y$, respectively. If every non-empty subset of $D$ and $E$ is dentable, then every non-empty subset of $D \times E$ in $X \times Y$ is dentable.

From the previous theorem, we can now prove that every space verifying the RNP has the Bishop-Phelps property. In fact, what Stegall proved was:

Corollary 3.2.20 Let $X$ and $Y$ be real or complex Banach spaces, and suppose that $X$ verifies the RNP, in its formulation that every non-empty bounded subset of $X$ is dentable. Let $C$ be a convex, closed, bounded subset of $X, T \in L(X, Y)$ and $\varepsilon>0$. Then, there exist an operator $S \in L(X, Y)$ such that $\|S-T\|<\varepsilon, S-T$ has rank one and the function $x \mapsto\|S x\|$ has a maximum in $C$.

And finally, if we put together all the results of this section, we obtain the following important corollary.

Corollary 3.2.21 Let $X$ be a real or complex Banach space. Then, the following facts are equivalent:

1. $X$ has the Bishop-Phelps property.

2. Every convex, balanced, closed and bounded subset of $X$ has the Bishop-Phelps property.

3. Every Banach space isomorphic to $X$ has property $A$.

4. $X$ has the Radon-Nikodym property.

In this corollary, we can find the most important information of this section. 1) $\Rightarrow 2$ ) $\Rightarrow 3$ )

${ }^{5}$ Let $X$ and $Y$ be Banach spaces and $U \subset X$ an open subset of $X$. A function $f: U \rightarrow Y$ is called Fréchet differentiable at $x \in U$ if there exists a bounded linear operator $T: X \rightarrow Y$ such that

$$
\lim _{h \rightarrow 0} \frac{\|f(x+h)-f(x)-T h\|_{Y}}{\|h\|_{X}}=0
$$

The limit here is meant in the usual sense of a limit of a function defined on a metric space. 
is evident. Bourgain [Bou97] proved the equivalence 2) $\Leftrightarrow 4$ ). Huff [Huf80] improved one of this implications, obtaining, in fact, 3) $\Rightarrow 4$ ). And finally, Stegall [Ste86] improved the other implication, proving 4$) \Rightarrow 1$ ).

With the information obtained in this section, we can find a new big class of Banach spaces $X$ that verify $\overline{N A(X)}=L(X)$. As we have already seen, if a Banach space $X$ has the RNP, then every Banach space isomorphic to $X$ has property $A$. In particular, we have the following result.

Result 3.2.22 Let $Y$ be a Banach space with the RNP. Then, for every $X$ isomorphic to $Y$,

$$
\overline{N A(X)}=L(X)
$$

Therefore, if we want to present some examples of spaces verifying $\overline{N A(X)}=L(X)$, it is enough to give some spaces with the RNP. In the section VII.7 of [DU77], they collect some examples with the RNP. We will further study them in the following sections, as we need too many new definitions to present most of them now. However, we can show now one of these examples. Banach spaces which are a separable dual of another Banach space have the RNP. Then, in particular, we have the following result.

Result 3.2.23 Let $Y$ be $\ell_{1}$ or $\ell_{p}$, with $1<p<\infty$. Then, for every $X$ isomorphic to $Y$,

$$
\overline{N A(X)}=L(X) \text {. }
$$

This result has also important consequences for the spaces that do not have the RNP. Among the classical Banach spaces, we can stand out $L^{1}(\Omega)$ and $L^{\infty}(\Omega)$, with $\Omega$ an open bounded subset of $\mathbb{R}^{n}$, and $C(K)$, for $K$ an infinite compact space, as examples of spaces without the Radon-Nikodym property. Then, for each one of them, called $X$, there exist two renormings, $X_{1}$ and $X_{2}$, such that

$$
\overline{N A\left(X_{1}, X_{2}\right)} \neq L\left(X_{1}, X_{2}\right)
$$

We will find specifically some of these renormings in the following chapter, where we present some counterexamples for $\overline{N A(X, Y)}=L(X, Y)$.

\subsubsection{Relation between $\overline{N A(X)}=L(X)$ AND the RNP}

In the previous subsections we have seen an important result which links measure theory with the theory of norm-attaining operators: A Banach space X has the Radon-Nikodym property if, and only if, every space isomorphic to $X$ has property $A$.

However, for a Banach space, the property that every space isomorphic to it has property $A$ is a very strong property. And it is also very difficult to check if a space has this property, as it involves infinite Banach spaces in the codomain, for infinite different spaces isomorphic to $X$. This suggests the possibility of weaken this property to a property which only involves the space of the domain, but still having an equivalence with the RNP.

For that reason, in this subsection we are going to study the relation between the following facts:

(i) $X$ has the RNP.

(ii) $\overline{N A(X)}=L(X)$ for every equivalent renorming of $X$. 
First, let us make the following remark. We have proved that if $X$ has the RNP, then every space isomorphic to $X$ has property $A$. In particular, if $Y$ is a space isomorphic to $X$, then $\overline{N A(Y)}=L(Y)$. And as every equivalent renorming of $X$ is isomorphic to $X$, we have (ii).

Therefore, we have to study in what cases $(i i) \Rightarrow(i)$. In the following result we prove that there exist some Banach spaces verifying this property.

Proposition 3.2.24 Let $Y$ be a Banach space and consider $X \cong Y \oplus Y$, where we are considering one of these norms:

- $X \cong Y \oplus_{1} Y \Rightarrow\|x\|_{X}=\left\|y_{1}\right\|_{Y}+\left\|y_{2}\right\|_{Y} \quad \forall x=\left(y_{1}, y_{2}\right)$.

- $X \cong Y \oplus_{\infty} Y \Rightarrow\|x\|_{X}=\max \left\{\left\|y_{1}\right\|_{Y},\left\|y_{2}\right\|_{Y}\right\} \quad \forall x=\left(y_{1}, y_{2}\right)$.

Then, if $X$ verifies $\overline{N A(X)}=L(X)$ for every equivalent norm, $X$ has the RNP.

Proof: Suppose that $X$ does not have the RNP. Then, in particular, $Y$ does not have the RNP. By the Huff theorem, Theorem 3.2.10, there exist norms $\|\cdot\|_{1}$ and $\|\cdot\|_{2}$ in $Y$, equivalent to the initial norm of $Y$, such that, if we denote $Y_{1}=\left(Y,\|\cdot\|_{1}\right)$ and $Y_{2}=\left(Y,\|\cdot\|_{2}\right)$, then

$$
\overline{N A\left(Y_{1}, Y_{2}\right)} \neq L\left(Y_{1}, Y_{2}\right) \text {. }
$$

Hence, by Theorem 3.2.13 we have $\overline{N A\left(Y_{1} \oplus Y_{2}\right)} \neq L\left(Y_{1} \oplus Y_{2}\right)$, so if we take $\tilde{X}=Y_{1} \oplus Y_{2}$, as $\tilde{X} \cong X$, there exists an equivalent renorming of $X$ that does not satisfy the property of the hypothesis, which is a contradiction.

We have to make a remark about this proof. In Theorem 3.2.13, we proved the result we are using in the previous proof for the case of the sum $\oplus_{\infty}$, i.e., the norm of the maximum. However, it is easy to see that this result also holds for the case of the norm $\|\cdot\|_{1}$.

In view of this result, an interesting question to ask is when a space is isomorphic to the square of another space (or even to the square of itself), i.e., when, for a Banach space $X$, there exists another Banach space $Y$ such that $X \cong Y \oplus Y$ (or $X \cong X \oplus X)$.

We will study now the situation $X \cong X \oplus X$. It is clear that finite dimensional spaces cannot verify this property, due to the different dimensions of $X$ and $X \oplus X$. However, for infinite dimensional spaces we do not have an argument of this kind.

Let us begin seeing two examples of spaces verifying this property.

- Example 3.2.25 $c \cong c \oplus c$

It is clear that $c_{0} \cong c_{0} \oplus c_{0}$ (given a sequence of $c_{0}$, it is enough to split it into the parts supported on the evens and odds).

Moreover, $c \cong c_{0}$. For proving that, consider

$$
\begin{aligned}
& T: \quad c \quad \longrightarrow \quad c_{0} \\
& x=\left\{x_{n}\right\} \quad \mapsto \quad T x \quad:=\left\{l(x), x_{1}-l(x), x_{2}-l(x), \ldots\right\},
\end{aligned}
$$

where $l(x)$ is the limit of the sequence $x=\left\{x_{n}\right\}$ (which exists, because $x \in c$ ). Then, $T$ is a topological isomorphism.

Consequently, $c \cong c \oplus c$. 
- Example 3.2.26 $C[0,1] \cong C[0,1] \oplus C[0,1]$

In the proof of this example we need the following two results, extracted from [Ban01].

Proposition 3.2.27 The space $C[0,1]$ is isomorphic to $C[0,1] \oplus c$.

Proof: Let $E$ denote the subspace of $C[0,1]$ consisting of the functions $f \in C[0,1]$ which satisfy the condition

$$
f\left(\frac{1}{n}\right)=0 \text { for } n=1,2, \ldots
$$

For each $f \in C[0,1]$, construct the function $\tilde{f}$ so that $\tilde{f}\left(\frac{1}{n}\right)=f\left(\frac{1}{n}\right)$ and which is linear in the intervals $\left[\frac{1}{n+1}, \frac{1}{n}\right]$ for every $n \in \mathbb{N}$.

Then, for every $f \in C[0,1]$, we associate the pair (consisting of a function and a sequence of numbers)

$$
\left(f(t),\left\{g\left(\frac{1}{n}\right)\right\}\right)
$$

where $f=g(t)-\tilde{g}(t)$. It is clear that $f \in E$ and $\left\{x\left(\frac{1}{n}\right)\right\} \in c$. And this correspondence defines a bounded linear operator.

Equally, we can see that for each pair $\left(f(t),\left\{x_{n}\right\}\right) \in E \oplus c$ there exists a continuous function $g$ such that $f(t)=g(t)-\tilde{g}(t)$ and $x_{n}=g\left(\frac{1}{n}\right)$ for $n \in \mathbb{N}$, from which it follows that the transformation under consideration is bijective between all $C[0,1]$ and all $E \oplus c$. Therefore, these two spaces are isomorphic.

Hence, the spaces $C[0,1] \oplus c$ and $E \oplus c^{2}$ are isomorphic. Now, as we have seen in the previous example that $c \cong c^{2}$, we have

$$
C[0,1] \oplus c \cong E \oplus c^{2} \cong E \oplus c \cong C[0,1] .
$$

Proposition 3.2.28 The space $C[0,1] \oplus C[0,1]$ is isomorphic to $C[0,1] \oplus \mathbb{R}$.

Proof: For each pair $(f(t), g(t)) \in C[0,1] \oplus C[0,1]$, we associate the pair $(h(t), x)$, where $h$ is the function defined by

$$
h(t)=\left\{\begin{array}{cc}
f(2 t) & \text { for } 0 \leq t \leq 1 / 2 \\
g(2 t-1)-g(0)+f(1) & \text { for } 1 / 2<t \leq 1
\end{array}\right.
$$

and $x$ is determined, for every $g \in C[0,1]$, by the value of $g(0)$.

Therefore, the space $C[0,1] \oplus C[0,1]$ is mapped to $C[0,1] \oplus \mathbb{R}$. And this transformation is a bounded linear operator. Also, as 


$$
f(t)=h\left(\frac{t}{2}\right) \quad \text { and } \quad y(t)=h\left(\frac{1}{2}+\frac{t}{2}\right)-h\left(\frac{1}{2}\right)+x
$$

the transformation is bijective. Thus, we have established the isomorphism between the spaces $C[0,1] \oplus C[0,1]$ and $C[0,1] \oplus \mathbb{R}$.

To finish with the proof of the example, observe that we have the following chain of isomorphisms

$$
\begin{gathered}
C[0,1] \oplus C[0,1] \stackrel{3.2 .28}{\cong} C[0,1] \oplus \mathbb{R} \stackrel{3.2 .27}{\cong}(C[0,1] \oplus c) \oplus \mathbb{R} \stackrel{c_{0} \cong c}{\cong}\left(C[0,1] \oplus c_{0}\right) \oplus \mathbb{R} \cong \\
C[0,1] \oplus\left(c_{0} \oplus \mathbb{R}\right) \cong C[0,1] \oplus c_{0} \stackrel{c_{0} \cong}{\cong} C[0,1] \oplus c \stackrel{3.2 .27}{\cong} C[0,1] .
\end{gathered}
$$

After these two positive examples, we are going to show now that not every infinite dimensional Banach space is isomorphic to its square. In fact, not every infinite dimensional Banach space is isomorphic to the square of another Banach space. However, before continuing showing examples for this, it is important to make the following remark: If $X^{*}$ is not isomorphic to its square, neither is $X$.

We will show now the results which appear in [CG97, Appendix 3.6]. Before introducing the first example of a space not isomorphic to its square, we need the following definition.

\section{Definition 3.2.29 - QUASI-REFLEXIVE SPACE.}

Let $X$ be a Banach space. Denote the dual space of $X$ by $X^{*}$, and the bidual space by $X^{* *}$, and consider for every $x \in X$ the canonical embedding, i. e., the function $J(x): X^{*} \rightarrow \mathbb{K}$ given by

$$
J(x)(f)=f(x) \quad f \in X^{*},
$$

as we did for defining reflexive spaces. Then $J(x) \in X^{* *}$. In fact, the image $J(X)$ is closed in $X^{* *}$, but it might not be equal to $X^{* *}$.

We saw that a Banach space $X$ is reflexive if it is linearly isometric to its bidual under the canonical embedding. We say that $X$ is quasi-reflexive (of order $d$ ) if the quotient $X^{\prime \prime} / J(X)$ has finite dimension $d$.

We can now enunciate the following result, published in [BP60].

Proposition 3.2.30 If $X$ is quasi-reflexive, non-reflexive and $Y$ is weakly sequentially complete, then $X$ and $X \oplus Y$ are not isomorphic to their squares.

Proof: The case of $X$ follows immediately from the fact that $X^{* *} / J(X)$ is finite dimensional, which implies that $X$ is not isomorphic to its square.

Now, observe that if we denote by $B_{1}(X)$ the subspace of those elements of $X^{* *}$ that are $\omega^{*}$-limits of sequences of elements of $X$, then

$$
\operatorname{dim} B_{1}(X \oplus Y) / X \oplus Y=\operatorname{dim} X^{* *} / X .
$$


Using this result, we can show an example of a space not isomorphic to its square.

\section{- Example 3.2.31 James space}

Let $\mathscr{P}$ denote the family of all finite increasing sequences of integers of odd length. If $p=\left(p_{1}, p_{2}, \cdots, p_{2 n+1}\right) \in \mathscr{P}$ and we consider $x=\left\{x_{n}\right\}$ a sequence of real numbers, we can define

$$
\|x\|_{p}:=\left(x_{p_{2 n+1}}^{2}+\sum_{m=1}^{n}\left(x_{p_{2 m-1}}-x_{p_{2 m}}\right)^{2}\right)^{1 / 2} .
$$

We define James space, denoted by $\mathbf{J}$, as the space of all the elements $x \in c_{0}$ satisfying the condition

$$
\|x\|:=\operatorname{Sup}\left\{\|x\|_{p}: p \in \mathscr{P}\right\}<\infty .
$$

James space serves as an example of the importance of the canonical embedding as the isomorphism between a space and its bidual for being reflexive, because it is isometrically isomorphic to its dual, while not being reflexive.

It is a non-reflexive space, as its image into its bidual under the canonical embedding has codimension one. Then, it is quasi-reflexive. Therefore, for the previous proposition, $\mathbf{J}$ is not isomorphic to its square.

\section{- Example 3.2.32 $\mathbf{J} \oplus C[0,1]$}

$\mathbf{J}^{*}$ is quasi-reflexive and $C[0,1]^{*}$ is weakly sequentially complete. Then, for the previous proposition, $\mathbf{J}^{*} \oplus C[0,1]^{*}$ is not isomorphic to its square. Therefore, $\mathbf{J} \oplus C[0,1]$ is also not isomorphic to its square.

Almost at the same time of the previous results, Z. Semadeni published [Sem60], where he showed the following result, more elaborated than the previous one.

Proposition 3.2.33 The space $C\left[0, \omega_{1}\right]$ is not isomorphic to its square.

A more complicated way to obtain spaces not isomorphic to its square is obtaining real Banach spaces admitting no complex structure. This was done by Szarek in [Sza86], where he showed that there exists an infinite dimensional superreflexive real Banach space that does not admit complex structure, what implies, in particular, that it is not isomorphic to the square of any Banach space.

Moreover, Gowers and Maurey showed in [GM97] that there exists a Banach space $X$ isomorphic to $X \oplus X \oplus X$, but not isomorphic to $X \oplus X$.

In [Kai95], Kaibkhanov proved that there exist Banach spaces $Y$ and $Z$ not isomorphic to their squares such that $Y \oplus Z$ is isomorphic to its square. This is also true for the space of Gowers and Maurey that we have just mentioned, as $X$ is not isomorphic to $X \oplus X$ but $X \oplus X$ is isomorphic to $X \oplus X \oplus X \oplus X$ (because $X$ is isomorphic to $X \oplus X \oplus X)$.

To sum up, remember that we are studying, for a Banach space $X$, the equivalence between the Radon-Nikodym property and the property that $\overline{N A(X)}=L(X)$ holds for every equivalent 
renorming of $X$. Indeed, we have proved that if $X$ is isomorphic to the square of a Banach space, then the previous equivalence holds. However, we have shown that even though many Banach spaces have this property, there also exist Banach spaces which are not isomorphic to any square of a Banach space.

Therefore, we do not know if the equivalence mentioned above is true in general. It is still an open question. We are going to present now the steps we could follow in order to try and prove this equivalence in general.

Let $X$ be an arbitrary Banach space verifying $\overline{N A(X)}=L(X)$ for every equivalent renorming of $X$. As we mentioned a couple of subsections before, in Theorem 3.2.9, Davis and Phelps proved the following characterization of the $R N P$ :

A Banach space $X$ has the RNP if, and only if, the unit ball of $X$, for every equivalent norm, is dentable.

Therefore, as the hypothesis we part of depends on every equivalent renorming of $X$, and this characterization also does, a fair strategy could be to try and proof that if $(X,\|\cdot\|)$ verifies

$$
\overline{N A((X,\|\cdot\|))}=L((X,\|\cdot\|))
$$

then $\mathbb{B}_{(X,\|\cdot\|)}$ is dentable. However, this property cannot be true, as $C[0,1]$ verifies $\overline{N A(C[0,1])}=$ $L(C[0,1])$ (see the following section) and $\mathbb{B}_{C[0,1]}$ is not dentable (proved in [DP74]). Another counterexample for this is $c_{0}$, because it has property $\beta$, so $\overline{N A\left(c_{0}\right)}=L\left(c_{0}\right)$, but $\mathbb{B}_{c_{0}}$ is not dentable (see, for example, [ABR03]).

Hence, even though we want to prove that $\mathbb{B}_{(X,\|\cdot\|)}$ is dentable for $(X,\|\cdot\|)$ an equivalent renorming of $X$, we cannot do it just using $\overline{N A((X,\|\cdot\|))}=L((X,\|\cdot\|))$. We need more information from other different renormings of $X$.

We can neither use a similar procedure to the one shown in the proof of Bourgain, Theorem 3.2.7. If we suppose that there exists a ball $\mathbb{B}_{(X,\|\cdot\|)}$ which is non-dentable, then we want to prove that there exists an equivalent norm of $\|\cdot\|$, called $|\cdot|$, such that $\overline{N A((X,|\cdot|))} \neq L((X,|\cdot|))$, obtaining a contradiction with the initial hypothesis. However, following a similar procedure to Bourgain, we can only obtain that there exist two norms $\|\cdot\|_{1}$ and $\|\cdot\|_{2}$ such that

$$
\overline{N A\left(\left(X,\|\cdot\|_{1}\right),\left(X,\|\cdot\|_{2}\right)\right)} \neq L\left(\left(X,\|\cdot\|_{1}\right),\left(X,\|\cdot\|_{2}\right)\right) .
$$

Therefore, we only obtain the desired result in case that $X \cong\left(X,\|\cdot\|_{1}\right) \oplus\left(X,\|\cdot\|_{2}\right)$, but we had already proved that result at the beginning of the subsection.

Hence, the equivalence

(i) $X$ has the RNP.

(ii) $\overline{N A(X)}=L(X)$ for every equivalent renorming of $X$.

is still, in general an open question.

\subsection{DENSITY OF NORM-ATtAINING OPERATORS BETWEen $C(K)$ AND $C(L)$}

In this section, we will consider the space of real-valued continuous functions defined on a compact, Hausdorff topological space $K$, which we denote by $C(K)$. Before enunciating the main results of this section, it is important to introduce some notation and basic properties. 
Let $K$ be a compact, Hausdorff topological space and $X$ a Banach space. Then, we will denote by $\omega^{*}\left(K, X^{*}\right)$ the Banach space of the applications $u: K \rightarrow X^{*}$ which are continuous in $X^{*}$ with the weak-* topology. This space has the following associated norm

$$
\|u\|=\sup \{\|u(x)\|: x \in K\}
$$

for every $u \in \omega^{*}\left(K, X^{*}\right)$ (as $K$ is a compact, Hausdorff topological space and $u$ is $\omega^{*}$-continuous, then the set $\{\|u(x)\|: x \in K\}$ is bounded).

We will represent by $C(K)$ the space of real-valued continuous functions on $K$. Then, it is clear that the application

$$
\Phi: L(X, C(K)) \rightarrow \omega^{*}\left(K, X^{*}\right)
$$

given by

$$
(\Phi(T)(k))(x)=(T x)(k), \forall k \in K, \forall x \in X
$$

is an isometric isomorphism. Then, we can consider the operators between $X$ and $C(K)$ as $\omega^{*}$ continuous functions, so we have the following charaterization for norm-attaining operators.

Proposition 3.3.1 An operator $T \in L(X, C(K))$ attains its norm if, and only if, there exists a point $k \in K$ such that

$$
\|\Phi(T)(k)\|=\|T\|
$$

and the functional $\Phi(T)(k)$ attains its norm.

We will also introduce the following characterization for norm-attaining functionals in $L^{1}[0,1]$, as we will need it in the next section.

Proposition 3.3.2 Let $T \in L\left(L^{1}[0,1], C(K)\right)$. A necessary and sufficient condition for $T$ to attain its norm is that there exists $k_{0} \in K$ and a subset $E$ in $[0,1]$ Lebesgue measurable such that

$$
\mu(E)>0 \text { and }\left|\psi\left(k_{0}\right)(t)\right|=\sup \{\|\psi(k)\|: k \in K\} \forall t \in E,
$$

where $\mu$ is the Lebesgue measure and, for every $k \in K, \psi(k)$ is the function of $L^{\infty}[0,1]$ associated to the functional $\Phi(T)(k)$ in the usual identification $L^{\infty}[0,1] \equiv\left(L^{1}[0,1]\right)^{*}$.

Given a locally compact, Hausdorff topological space $\Omega$, we define by $C_{0}(\Omega)$ the space of continuous functions $f: \Omega \rightarrow \mathbb{R}$ which vanish at infinity, i.e., for every $\varepsilon>0$, the set

$$
\{\omega \in \Omega:|f(\omega)| \geq \varepsilon\}
$$

is compact. Considering in this space the uniform norm, it is complete.

Now, we can use the following theorem to characterize the dual of this space. 


\section{Theorem 3.3.3 - RIESZ-MARKOV THEOREM.}

Let $\Omega$ be a locally compact, Hausdorff topological space. For every bounded linear functional $\psi \in C_{0}(\Omega)$, there exists a unique regular countably additive complex Borel measure $\mu$ on $\Omega$ such that

$$
\psi(f)=\int_{\Omega} f(x) d \mu(x)
$$

for all $f \in C_{0}(\Omega)$. The norm of $\psi$ as a linear functional is the total variation of $\mu$, that is,

$$
\|\psi\|=|\mu|(\Omega) .
$$

Moreover, $\psi$ is positive if and only if the measure $\mu$ is non-negative.

We will introduce now some results which make the proof of the density of norm-attaining operators between two spaces of the kind $C(K)$ easier. For that, as mentioned above, we will work with functions $\omega^{*}$-continuous instead of operators.

Lemma 3.3.4 Let $K$ be a compact, Hausdorff topological space and $V$ an open subset of $K$. Then, the mapping from $\mathscr{M}(K)$ to $\mathbb{R}$ defined by

$$
\mu \mapsto|\mu|(V)
$$

is $\omega^{*}$-lower semicontinuous. ${ }^{a}$

${ }^{a}$ Let $X$ be a topological space, $x_{0} \in X$ and $f: X \rightarrow \mathbb{R} \cup\{-\infty,+\infty\}$ an extended real-valued function. We say that $f$ is lower semicontinuous at $x_{0}$ if for every $\varepsilon>0$ there exists a neighborhood $U$ of $x_{0}$ such that

- $f(x) \geq f\left(x_{0}\right)-\varepsilon$ for all $x \in U$ when $f\left(x_{0}\right)<+\infty$

- $f(x)$ tends to $+\infty$ as $x$ tends towards $x_{0}$ when $f\left(x_{0}\right)=+\infty$

Equivalently, this can be expressed as

$$
\liminf _{x \rightarrow x_{0}} f(x) \geq f\left(x_{0}\right)
$$

The only tool used in the proof of this result is Theorem 3.3.3 to get an element of $C_{0}(V)$ and extend it to an element of $C(K)$ which vanishes out of $V$. As an immediate consequence of this result we have the following one.

Lemma 3.3.5 Let $K, L$ be compact, Hausdorff topological spaces and $\mu: K \rightarrow \mathscr{M}(L) \omega^{*}$ continuous. Take $\varepsilon>0, k_{0} \in K$ and $V$ an open subset of $L$. Then, there exists an open neighbourhood of $k_{0}, U$, such that

$$
|\mu(k)|(V)>\left|\mu\left(k_{0}\right)\right|(V)-\varepsilon, \forall k \in U .
$$

Its proof, as mentioned above, is an immediate consequence of the previous lemma and the fact that $\mu$ is $\omega^{*}$-continuous.

The last tools we need in the proof of the main result of this section are the following ones.

Lemma 3.3.6 For $K, L$ as in the previous lemma, let $\mu: K \rightarrow \mathscr{M}(L)$ be a $\omega^{*}$-continuous function and $\delta>0$. Then, there exist an $\omega^{*}$-continuous function $\mu^{\prime}: K \rightarrow \mathscr{M}(L), U$ an open subset of $K, V$ an open subset of $L$ and a function $h \in C(L)$ such that 
(i) $\left|\mu^{\prime}(k)\right|(V)=0, \forall k \in U$.

(ii) $\int_{L} h d \mu^{\prime}(k) \geq\left\|\mu^{\prime}\right\|-\delta, \forall s \in U$.

(iii) $\|h\|=1$ and $|h(t)|=1$ if $t \in K \backslash V$.

(iv) $\left\|\mu-\mu^{\prime}\right\| \leq \delta$.

Lemma 3.3.7 Let $\mu: K \rightarrow \mathscr{M}(L)$ be a $\omega^{*}$-continuous function, $\varepsilon>0, U$ an open subset of $K, V$ an open subset of $L, k_{0} \in U$ and a function $h \in C(L)$ such that

(a) $|\mu(k)|(V)=0, \forall k \in U$,

(b) $\int_{L} h d \mu\left(k_{0}\right) \geq\|\mu\|-\varepsilon$,

(c) $\|h\|=1$ and $|h(t)|=1$ if $t \in K \backslash V$.

Then, given $r>2 / 3$, there exist a $\omega^{*}$-continuous function $\mu^{\prime}: K \rightarrow \mathscr{M}(L)$ and an element $k_{1} \in U$ verifying

(i) $\left|\mu^{\prime}(k)\right|(V)=0, \forall k \in U$,

(ii) $\int_{L} h d \mu^{\prime}\left(k_{1}\right) \geq\left\|\mu^{\prime}\right\|-r \varepsilon$,

(iii) $\left\|\mu-\mu^{\prime}\right\| \leq r \varepsilon$.

Once we have introduced all these results, whose proof can be found in [Rui94], we can enunciate and demonstrate the result of density of norm-attaining operators between $C(K)$ and $C(L)$.

Theorem 3.3.8 Let $K, L$ be two compact, Hausdorff topological spaces. Then, the set $N A(C(L), C(K))$ is dense in $L(C(L), C(K))$.

Proof: Let $\varepsilon>0$ and $T \in L(C(L), C(K))$. Consider its representation in $\omega^{*}(K, \mathscr{M}(L))$ as $\mu: K \rightarrow \mathscr{M}(L)$.

Take now $2 / 3<r<1$ and $\eta>0$ such that $\frac{\eta}{1-r}<\varepsilon$. Then, Lemma 3.3 .6 provides a $\omega^{*}$ continuous function $\mu_{0}: K \rightarrow \mathscr{M}(L), U$ an open subset of $K, V$ an open subset of $L$ and a function $h \in C(L)$ verifying the four properties in the enunciate of the lemma.

Fixing an element $k_{0} \in U$, Lemma 3.3.7 guarantees the existence of a function $\mu_{1}: K \rightarrow$ $\mathscr{M}(L)$ and $k_{1} \in U$ such that

$$
\begin{gathered}
\left|\mu_{1}(k)\right|(V)=0, \forall k \in U \\
\left\|\mu_{0}-\mu_{1}\right\| \leq r \eta \\
\int_{L} h d \mu_{1}\left(k_{1}\right) \geq\left\|\mu_{1}\right\|-r \eta
\end{gathered}
$$

Repeating this procedure, we can obtain by recurrence two sequences $\left\{\mu_{n}\right\}_{n \in \mathbb{N}} \subseteq \omega^{*}(K, \mathscr{M}(L))$ and $\left\{k_{n}\right\}_{n \in \mathbb{N}}$ verifying

$$
\begin{gathered}
\left\|\mu_{n-1}-\mu_{n}\right\| \leq r^{n} \eta, \\
\int_{L} h d \mu_{n}\left(k_{n}\right) \geq\left\|\mu_{n}\right\|-r^{n} \eta .
\end{gathered}
$$

Then, if for every $n \in \mathbb{N}$ we consider $T_{n}$ the operator represented by the function $\mu_{n}$, the sequence $\left\{T_{n}\right\}_{n \in \mathbb{N}}$ is a Cauchy sequence, so it converges to an operator $\hat{T} \in L(C(L), C(K))$. Now we are going to show that this operator attains its norm and is really close to $T$. 
Consider $\tau$ as the $\omega^{*}$-continuous function which represents the operator $\hat{T}$. Then, for what we have seen before,

$$
\hat{T}(h)\left(k_{n}\right)=\int_{L} h d \tau\left(k_{n}\right) \geq \int_{L} h d \mu_{n}\left(k_{n}\right)-\left\|\tau-\mu_{n}\right\| \geq\left\|\mu_{n}\right\|-r^{n} \eta-\left\|\tau-\mu_{n}\right\| .
$$

However,

$$
\lim _{n \rightarrow \infty} r^{n} \eta+\left\|\tau-\mu_{n}\right\|=0
$$

Then, if we take limits in the previous expression, we obtain

$$
\|\hat{T}(h)\| \geq\|\tau\|=\|\hat{T}\|,
$$

so $\hat{T}$ attains its norm at $h$ and

$$
\|T-\hat{T}\|=\|\mu-\tau\| \leq\left\|\mu-\mu_{0}\right\|+\sum_{n=1}^{\infty}\left\|\mu_{n}-\mu_{n-1}\right\| \leq \eta+\sum_{n=1}^{\infty} r^{n} \eta=\frac{\eta}{1-r}<\varepsilon .
$$

It is clear that, as a consequence of this theorem, we have the following result.

Result 3.3.9 Let $K$ be a compact, Hausdorff topological space. Then,

$$
\overline{N A(C(K))}=L(C(K)) \text {. }
$$

\subsubsection{C(K) SPACES WITH PROPERTY A}

In general, if $K$ is a compact, Hausdorff topological space, $C(K)$ might not have property $A$. In this subsection we are going to prove that the space $C(K)$ has property $A$ if, and only if, $K$ is finite.

For that, we need to remember a result that we enunciated when we defined property $A$. We saw in Section 3.1.1 that if $X$ has a unit ball which is the closed convex hull of a set of uniformly strongly exposed points, then $X$ has property $A$. Next, we also mentioned a pseudoreciprocal result for this one in the separable case, which states that if $X$ is separable and verifies property $A$, then the unit ball of $X$ is the absolutely closed convex hull of its strongly exposed points (Proposition 3.1.16).

As a corollary of this result, we are going to prove the following proposition.

Proposition 3.3.10 Let $K$ be a compact metric space. Then, the space $C(K)$ has property $A$ if, and only if, $K$ is finite.

Proof: As $K$ is a compact metric space, the space $C(K)$ is separable. We are going to prove that, if $K$ is infinite, the unit ball of $C(K)$ lacks extreme points.

Suppose that $f \in \mathbb{S}_{C(K)}$ is an extreme point of $C(K)$. Then, let $\mu \in \mathscr{M}(K)$ be the measure which exposes strongly $\mathbb{B}_{C(K)}$ at $f$. Therefore,

$$
1=\int_{K} f d \mu
$$


and there exists $\delta>0$ such that

$$
\|f-g\|<1
$$

when

$$
\left|\operatorname{Re} \int_{K}(f-g) d \mu\right|<\delta
$$

For being $K$ infinite and $\mu$ regular, there exists an open nonempty set $G$ such that $0<$ $|\mu|(G)<\delta / 2$. Using Urysohn's Lemma ${ }^{6}$, it is easy to find a function $g \in C(K)$ such that

$$
\|g\| \leq 1,\|f-g\|=2 \text {, and } g(t)=f(t), \forall t \in K \backslash G .
$$

However,

$$
\left|\int_{K}(f-g) d \mu\right|=\left|\int_{G}(f-g) d \mu\right| \leq|\mu|(G)\|f-g\|<\delta,
$$

that implies $\|f-g\|<1$, which is a contradiction.

Let $K$ now be infinite. Even though $C(K)$, for what we have just proved, does not have property $A$, we can find some Banach spaces $Y$ such that $\overline{N A(C(K), Y)}=L(C(K), Y)$.

For that, we need the following theorem, whose proof we are not showing here, but can be found in [Rui94].

Theorem 3.3.11 Let $K$ be a compact, Hausdorff topological space and $Y$ a Banach space. Then, given $T \in L(C(K), Y)$ weakly compact, and $\varepsilon>0$, there exists an operator $S \in$ $N A(C(K), Y)$ such that

$$
\|T-S\|<\varepsilon
$$

From this result, we can obtain some consequences. Firstly, remember that we have already seen that if $X$ is reflexive and $T \in L(X, Y)$, with $Y$ arbitrary, then $T\left(\mathbb{B}_{X}\right)$ is weakly compact, so $T$ is a weakly compact operator. Then, we have the following result.

Corollary 3.3.12 Let $K$ be a compact, Hausdorff topological space and $Y$ a reflexive, real Banach space. Then,

$$
\overline{N A(C(K), Y)}=L(C(K), Y)
$$

In particular,

\footnotetext{
${ }^{6}$ Two disjoint closed subsets $A$ and $B$ of a topological space $X$ are said to be separated by neighbourhoods if there exist neighbourhoods $U$ of $A$ and $V$ of $B$ that are also disjoint.

$A$ and $B$ are said to be separated by a function if there exists a continuous function $f$ from $X$ into the unit interval $[0,1]$ such that $f(a)=0$ for all $a \in A$ and $f(b)=1$ for all $b \in B$. Any such function is called a Urysohn function for $A$ and $B$.

A normal space is a topological space in which any two disjoint closed sets can be separated by neighbourhoods.

Urysohn's Lemma states that: A topological space is normal if, and only if, any two disjoint closed sets can be separated by a continuous function.
} 


$$
\overline{N A\left(C[0,1], \ell_{2}\right)}=L\left(C[0,1], \ell_{2}\right) \text {. }
$$

We can also deduce from the previous theorem this result:

Corollary 3.3.13 Let $Y$ be a Banach space which does not contain an isomorphic copy of $c_{0}$, and let $K$ be a compact, Hausdorff topological space. Then,

$$
\overline{N A(C(K), Y)}=L(C(K), Y) \text {. }
$$

In particular,

$$
\overline{N A\left(C[0,1], L^{1}[0,1]\right)}=L\left(C[0,1], L^{1}[0,1]\right) .
$$

Proof: In this case, every operator is weakly compact, something we can find in [DU77]. Moreover, $L^{1}$ does not contain isomorphic copies of $c_{0}$. 


\subsection{SUMMARY}

Positive Results FOR $\overline{N A(X, Y)}=L(X, Y) \quad(\overline{X Y})$

\begin{tabular}{|c|c|c|c|}
\hline$X$ & $Y$ & RESULT & COMMENTARIES \\
\hline Property A & All & Definition 3.1.1 & \\
\hline All & Property B & Definition 3.1 .2 & \\
\hline Property $\alpha$ & All & Theorem 3.1.17 & Example: $\ell_{1}$ \\
\hline All & Property $\beta$ & Theorem 3.1 .12 & $\begin{array}{l}\text { Examples: } c_{0}, c, \ell_{\infty}, C_{b}(L), \\
\text { finite dim. space whose unit ball } \\
\text { is a polyhedron }\end{array}$ \\
\hline $\begin{array}{l}\text { Its unit ball is the closed } \\
\text { convex hull of a set of unif. } \\
\text { strongly exposed points }\end{array}$ & All & Theorem 3.1 .14 & $\begin{array}{l}\text { Example: } \mathbb{R}^{2} \text { with the norm } \\
\text { whose unit ball is } \mathbb{B}_{2}+\mathbb{B}_{\infty}\end{array}$ \\
\hline Property quasi- $\alpha$ & All & Theorem 3.1.44 & Example 3.1 .48 \\
\hline All & Property quasi- $\beta$ & Theorem 3.1.38 & Example 3.1 .42 \\
\hline All & $\begin{array}{c}Y \text { renormed } \\
\text { with property } \beta\end{array}$ & Partington th. & $\begin{array}{l}\text { Every Banach space can be } \\
\text { renormed to verify property } \beta\end{array}$ \\
\hline $\begin{array}{c}X \text { renormed } \\
\text { with property } \alpha\end{array}$ & All & Schachermayer th. & $\begin{array}{l}\text { Every weakly compactly } \\
\text { generated Banach space can be } \\
\text { renormed to verify property } \alpha\end{array}$ \\
\hline $\begin{array}{c}X \text { renormed } \\
\text { with property } \alpha\end{array}$ & All & $\begin{array}{l}\text { Godun- } \\
\text { Troyanski th. }\end{array}$ & $\begin{array}{l}\text { Every Banach space with a biorthogonal } \\
\text { system with cardinality equal to dens } X \\
\text { can be renormed to verify property } \alpha\end{array}$ \\
\hline $\begin{array}{l}X \text { is isomorphic to } \\
\text { a space with the RNP }\end{array}$ & All & Corollary 3.2.21 & $\begin{array}{l}\text { A Banach space has the RNP, if, and } \\
\text { only if, every Banach space isomorphic } \\
\text { to it has property } A\end{array}$ \\
\hline$X$ has the BPp & All & Definition 3.2 .5 & \\
\hline
\end{tabular}




\begin{tabular}{|c|c|c|c|}
\hline$X$ & $Y$ & RESULT & COMMENTARIES \\
\hline $\begin{array}{l}\qquad C(K) \\
\text { with } K \text { a compact, } \\
\text { Hausdorff top. space }\end{array}$ & $\begin{array}{l}\qquad C(L) \\
\text { with } L \text { a compact, } \\
\text { Hausdorff top. space }\end{array}$ & Theorem 3.3.8 & \\
\hline $\begin{array}{c}C(K) \\
\text { with } K \text { finite }\end{array}$ & All & Proposition 3.3 .10 & $\begin{array}{l}\text { This result is a characterization: } \\
\text { If } \overline{N A(C(K), Y)}=L(C(K), Y) \\
\text { for every } Y, K \text { is finite }\end{array}$ \\
\hline $\begin{array}{l}\qquad C(K) \\
\text { with } K \text { a compact, } \\
\text { Hausdorff top. space }\end{array}$ & $\begin{array}{c}\text { Reflexive } \\
\text { and real }\end{array}$ & Corollary 3.3 .12 & $\begin{array}{l}\frac{\text { Example: }}{N A\left(C[0,1], \ell_{2}\right)}=L\left(C[0,1], \ell_{2}\right)\end{array}$ \\
\hline $\begin{array}{l}\qquad(K) \\
\text { with } K \text { a compact, } \\
\text { Hausdorff top. space }\end{array}$ & $\begin{array}{l}\text { Does not contain an } \\
\text { isomorphic copy of } c_{0}\end{array}$ & Corollary 3.3.13 & $\begin{array}{c}\frac{\text { Example: }}{N A\left(C[0,1], L^{1}[0,1]\right)}=L\left(C[0,1], L^{1}[0,1]\right)\end{array}$ \\
\hline
\end{tabular}

Positive Results for $\overline{N A(X)}=L(X)$

\begin{tabular}{|c|c|c|}
\hline$X$ & RESULT & COMMENTARIES \\
\hline Property A & Definition 3.1.1 & \\
\hline Property B & Definition 3.1.2 & \\
\hline Property $\alpha$ & Theorem 3.1.17 & Example: $\ell_{1}$ \\
\hline Property $\beta$ & Theorem 3.1.12 & $\begin{array}{l}\text { Examples: } c_{0}, c, \ell_{\infty}, C_{b}(L), \\
\text { finite dim. space whose unit ball } \\
\text { is a polyhedron }\end{array}$ \\
\hline $\begin{array}{l}\text { Its unit ball is the closed } \\
\text { convex hull of a set of unif. } \\
\text { strongly exposed points }\end{array}$ & Theorem 3.1.14 & $\begin{array}{l}\text { Example: } \mathbb{R}^{2} \text { with the norm } \\
\text { whose unit ball is } \mathbb{B}_{2}+\mathbb{B}_{\infty}\end{array}$ \\
\hline Property quasi- $\alpha$ & Theorem 3.1.44 & Example 3.1 .42 \\
\hline Property quasi- $\beta$ & Theorem 3.1.38 & Example 3.1 .48 \\
\hline
\end{tabular}




\begin{tabular}{|c|c|c|}
\hline$X$ & RESULT & COMMENTARIES \\
\hline Has the RNP & Corollary 3.2.21 & $\begin{array}{c}\text { Examples: } \ell_{1}, \ell_{p} \text { with } 1<p<\infty \\
\text { In Proposition } 3.2 .24 \text { we can see a } \\
\text { semi-converse result: If } X \cong Z \oplus Z \text { and } \overline{N A(X)}=L(X) \\
\text { for every equiv. renorming, } X \text { has the RNP }\end{array}$ \\
\hline Has the BPp & Definition 3.2.5 & \\
\hline $\begin{array}{l}\qquad C(K) \\
\text { with } K \text { a compact, } \\
\text { Hausdorff top. space }\end{array}$ & Theorem 3.3.8 & \\
\hline
\end{tabular}

NEGATIVE RESULTS FOR $\overline{N A(X, Y)}=L(X, Y) \quad(\overline{X Y})$

\begin{tabular}{|c|c|c|c|}
\hline$X$ & $Y$ & RESULT & COMMENTARIES \\
\hline \hline$X_{1}=\left(X,\|\cdot\|_{1}\right)$ & $X_{2}=\left(X,\|\cdot\|_{2}\right)$ & & If $X$ does not have the RNP, \\
\cline { 1 - 1 } $\begin{array}{c}\text { where }(X,\|\cdot\|) \text { does not have the RNP } \\
\text { and }\|\cdot\|_{1},\|\cdot\|_{2} \text { are equivalent to }\|\cdot\|\end{array}$ & Huff th. & $\begin{array}{c}\text { there exist certain equiv. renormings of } X, \\
X_{1} \text { and } X_{2}, \text { such that } \overline{N A\left(X_{1}, X_{2}\right)} \neq L\left(X_{1}, X_{2}\right)\end{array}$ \\
\hline
\end{tabular}

NEGATIVE RESULTS FOR $\overline{N A(X)}=L(X) \quad(\bar{X})$

\begin{tabular}{|c|c|c|}
\hline$X$ & RESULT & COMMENTARIES \\
\hline $\begin{array}{l}\qquad X=Y_{1} \oplus_{\infty} Y_{2} \\
\text { where }(Y,\|\cdot\|) \text { does not have the RNP, } \\
\qquad Y_{1}=\left(Y,\|\cdot\|_{1}\right) \text { and } Y_{2}=\left(Y,\|\cdot\|_{2}\right) \\
\text { with }\|\cdot\|_{1},\|\cdot\|_{2} \text { equivalent to }\|\cdot\|\end{array}$ & $\begin{array}{l}\text { Huff theorem } \\
\quad+ \\
\text { Theorem 3.2.13 }\end{array}$ & \\
\hline
\end{tabular}



COUNTEREXAMPLES BASED ON LINDENSTRAUSS

ARGUMENT

GOWERS' COUNTEREXAMPLE

NO STRICTLY CONVEX INFINITE-DIMENSIONAL

BANACH SPACE HAS PROPERTY B

OTHER COUNTEREXAMPLES

SUMMARY

\section{COUNTEREXAMPLES FOR $\overline{N A(X, Y)}=L(X, Y)$}

In this chapter, we are going to present some new counterexamples for the problem of density of norm-attaining operators, i.e., we are going to show some examples of Banach spaces $X$ and $Y$ such that $\overline{N A(X, Y)} \neq L(X, Y)$.

In particular, in virtue of Theorem 3.2.13, from these examples we will also obtain some examples of Banach spaces $X$ such that $\overline{N A(X)} \neq L(X)$.

We will begin presenting some counterexamples based on Lindenstrauss' argument, i.e., the procedure he followed to show that if $Y$ is a strictly convex Banach space, then $N A\left(c_{0}, Y\right)$ is not dense in $L\left(c_{0}, Y\right)$. First, we will present Gowers' counterexample, which consists on the construction of a space (afterwards called Gowers' space) $G$ such that if $Y$ is a strictly convex Banach space and $T$ from $G$ to $Y$ attains its norm, then $T$ is a finite rank operator. Therefore, if there exists a non-compact operator from $G$ to $Y$, we have a counterexample for the problem of density of norm-attaining operators.

Later, we will show another counterexample based on Lindenstrauss' argument, which constitutes a generalization of the two counterexamples mentioned above. In this counterexample, M. Acosta showed that no strictly convex infinite-dimensional Banach space has property $B$. Therefore, her paper provides new examples of classical Banach spaces without property $B$.

Finally, we will present other counterexamples which are not based on Lindenstrauss' argument. We will discuss an example constructed by Johnson and Wolfe of a compact, metric space $K$ such that $\overline{N A\left(L^{1}[0,1], C(K)\right)} \neq L\left(L^{1}[0,1], C(K)\right)$. For that, we will need the results already presented in Section 3.3. This counterexample is inspired on another one of Schachermayer, for the couple $\left(L^{1}[0,1], C[0,1]\right)$, which is more extense and complicated.

\section{1 COUNTEREXAMPLES BASED ON LINDENSTRAUSS' ARGUMENT}

The examples presented in this section are constructed using a method based in the one that Lindenstrauss used to show that if $Y$ is a strictly convex Banach space, then $N A\left(c_{0}, Y\right)$ is not dense in $L\left(c_{0}, Y\right)$ (see proposition 2.2.21).

Using the same tools that the ones appearing in the proof of that proposition, we can obtain, in general, the following result, which will be essential in the construction of the counterexamples 
of this section:

Lemma 4.1.1 Let $X, Y$ be Banach spaces. Suppose that $Y$ is strictly convex and for every $x_{0} \in \mathbb{S}_{X}$ the closed linear span of the set of those $x \in X$ such that $\left\|x_{0} \pm x\right\| \leq 1$ has finite codimension. Then, $N A(X, Y) \subseteq F(X, Y)$.

Remember that for the case of $X=c_{0}$ we saw that if $x_{0} \in \mathbb{S}_{c_{0}}$, there exists $N \in \mathbb{N}$ such that $\left\|x_{0} \pm \frac{1}{2} e_{n}\right\| \leq 1$ for every $n \geq N$, a particular case of this lemma.

We will begin with the counterexample that W. Gowers published in 1990. This result is very important, as it shows, in particular, that $\ell_{p}$ does not have property $B$, for $1<p<\infty$.

\subsubsection{GOWERS' COUNTEREXAMPLE}

Even though we already know quite a bit about property $A$ from the things we have studied in the previous chapter, property $B$ is still quite unknown. Isomorphically, we know, by the Partington theorem, that every Banach space can be renormed with property $\beta$, implying property $B$. However, isometrically, we know very little about the problem.

We already know, by the Huff theorem, that if every equivalent renorming of a Banach space $X$ verifies property $B$, then $X$ has the RNP. The reciprocal affirmation is not true. In 1990, W. Gowers published in [Gow90] an easy demonstration that the separable Hilbert space, $\ell_{2}$, does not have property $B$. In fact, for every $1<p<\infty, \ell_{p}$ does not have property $B$. In his demonstration, he gives a stronger result: If a Banach space $Y$ is strictly convex and contains isometrically $\ell_{p}$, with $1<p<\infty$, then $Y$ does not have property $B$.

The idea of this counterexample is similar to the one used by Lindenstrauss. If $Y$ is a strictly convex Banach space, $X$ an arbitrary Banach space and $T$ an injective operator from $X$ to $Y$ which attains its norm in $x_{0} \in \mathbb{B}_{X}$, then $x_{0}$ is an extreme point of $\mathbb{B}_{X}$. Hence, for $Y=\ell_{p}$, with $1<p<\infty$, we will consider a space $X$ without extreme points in $\mathbb{B}_{X}$ such that there exists an injective operator from $X$ to $\ell_{p}$ that is hardly approximated by injective operators. We will see that we can use the same $X$ for every $p$.

The formalism used by Gowers for defining its space is unnecessarily complicated. We will show here another formulation, extracted from [Pay93].

\section{Definition 4.1.2 - GOWERS SPACE.}

Let us consider $\ell_{\infty}$, the space of the scalar bounded sequences. Consider $\left\{\varphi_{n}\right\}$ a sequence of norms that are equivalent to the usual one. Given a finite subset $J$ of $\mathbb{N}$, we denote by $|J|$ the number of elements in $J$, and $\left\{H_{n}\right\}$ will be the sequence of partial sums of the harmonic series, i.e., $H_{n}=\sum_{k=1}^{n} k^{-1}$. Then, for $n \in \mathbb{N}$ and $x \in \ell_{\infty}$, we define

$$
\varphi_{n}(x)=\frac{1}{H_{n}} \sup \left\{\sum_{j \in J}|x(j)|: J \subset \mathbb{N},|J|=n\right\} .
$$

It is clear that $\varphi_{n}$ is equivalent to the usual norm $\|\cdot\|_{\infty}$. In fact, we have

$$
\frac{1}{H_{n}}\|x\|_{\infty} \leq \varphi_{n}(x) \leq \frac{n}{H_{n}}\|x\|_{\infty} \quad\left(x \in \ell_{\infty}\right),
$$

and, in particular, $\varphi_{1}=\|\cdot\|_{\infty}$. The Gowers space is, by definition, the vectorial subspace of $\ell_{\infty}$ 
given by

$$
G=\left\{x \in \ell_{\infty}: \lim _{n \rightarrow \infty} \varphi_{n}(x)=0\right\}
$$

with the norm

$$
\|x\|=\max \left\{\varphi_{n}(x): n \in \mathbb{N}\right\} \quad(x \in G) .
$$

This space verifies the properties from the following theorem.

\section{Theorem 4.1.3}

1. $G$ is a Banach space.

2. If, for every $n \in \mathbb{N}, e_{n}$ is the sequence defined by $e_{n}(n)=1, e_{n}(k)=0$ for $k \neq n$, then the sequence $\left\{e_{n}\right\}$ is a Schauder basis for $G$.

3. The unit ball of $G$ lacks extreme points. Moreover, if $x \in G$ and $\|x\|=1$, we can find $\delta>0$ and $m \in \mathbb{N}$ such that for $n \geq m$ we have $\left\|x \pm \delta e_{n}\right\| \leq 1$.

4. For $1<p<\infty, G \subset \ell_{p}$ (as vectorial space) and the formal identity from $G$ to $\ell_{p}$ is a continuous linear operator.

We are not proving any of these properties here, as their proofs are long and tedious; for consulting them, see [Pay93]. As a consequence of these properties, we get the following corollary.

Corollary 4.1.4 If $Y$ is a strictly convex Banach space and $T \in N A(G, Y)$, then there exists a natural number $m$ such that $T\left(e_{n}\right)=0$ for $n \geq m$. In particular, $T$ is a finite rank operator.

Proof: Suppose, without loss of generality, that $1=\|T\|=\left\|T x_{0}\right\|$, with $x_{0} \in \mathbb{S}_{G}$. Let $\delta>0$ and $m \in \mathbb{N}$ be such that, for every $n \geq m$, we have $\left\|x_{0} \pm \delta e_{n}\right\| \leq 1$. Hence,

$$
\left\|T\left(x_{0} \pm \delta e_{n}\right)\right\| \leq\|T\|=\left\|T x_{0}\right\|,
$$

and the strict convexity of $Y$ implies $T\left(e_{n}\right)=0$ for every $n \geq m$. Since the subspace generated by $\left\{e_{n}\right\}$ is dense in $G$, the image of $G$ is contained in the finite-dimensional subspace of $Y$ generated by $\left\{T e_{n}\right\}_{1 \leq n<m}$.

As a consequence of this corollary, if there exists a non-compact operator from $G$ to $Y$, then $N A(G, Y)$ is not dense in $L(G, Y)$. Hence, $Y$ does not have property $B$.

In particular, in virtue of Theorem 3.2.13, we have the following result.

Result 4.1.5 Let $Y$ be a strictly convex Banach space such that there exists a non-compact operator from $G$ to $Y$ and consider $X=Y \oplus_{\infty} G$. Then,

$$
\overline{N A(X)} \neq L(X)
$$

If we considered $c_{0}$ instead of $G$, the previous corollary was already known by Lindenstrauss, see Proposition 2.2.21. The greatest difference between these two results is that while every bounded linear operator from $c_{0}$ to a reflexive 
Banach space is compact, the fourth statement of the previous theorem provides non-compact operators from $G$ to $\ell_{p}$ for $1<p<\infty$.

From the construction of $G$, we can see that the identity operator between $G$ and $\ell_{p}$ cannot be compact (if it were compact, the canonical basis of $\ell_{p}$ would have a partial sequence convergent in norm). Then, we have the following corollary.

Corollary 4.1.6 If $Y$ is a strictly convex Banach space containing a subspace which is isomorphic to $\ell_{p}$ for any $1<p<\infty$, then $Y$ does not have property $B$.

\subsubsection{NO STRICTLY CONVEX INFINITE-DIMENSIONAL BANACH SPACE HAS PROPERTY B}

In this section, we show another counterexample for $\overline{N A(X, Y)}=L(X, Y)$ based on Lindenstrauss' argument. This one was published by M. D. Acosta in [Aco99a]. In fact, it is a generalization of the two previous results by Lindenstrauss and Gowers, as the author shows that no strictly convex infinite-dimensional Banach space has property $B$, i.e., if $Y$ is a strictly convex Banach space with infinite dimension we can find a Banach space $X$ such that $\overline{N A(X, Y)} \neq L(X, Y)$.

This paper provides new examples of classical Banach spaces without property $B$, like the Hardy space $H^{1}$, any complex infinite-dimensonal $L^{1}(\mu)$, or, in general, any infinite-dimensional predual of a von Neumann algebra. The result for $L^{1}(\mu)$ partly answers the question posed by Johnson and Wolfe in [JW79] about $L^{1}(\mu)$ having property $B$, as it is shown that the answer is negative, at least, for the complex infinite-dimensional cases.

Firstly, the author introduces the Banach space she is going to use as the domain space. For that, let us assume that $\left\{\omega_{n}\right\}$ is a decreasing sequence of positive real numbers such that $\omega=\left\{\omega_{n}\right\} \notin \ell_{1}$ and $\omega_{1}<1$.

Consider now the Banach space $Z$ of the sequences of scalars with norm:

$$
\|z\|:=\|(1-\omega) z\|_{\infty}+\|\omega z\|_{1}<\infty
$$

for every $z \in Z$, where $\|\cdot\|_{1}$ and $\|\cdot\|_{\infty}$ are the usual norms of $\ell_{1}$ and $\ell_{\infty}$, respectively. Also, denote by $\left\{e_{n}\right\}$ the sequence of functionals given by

$$
e_{n}(z)=z(n)
$$

It is clear that $e_{n} \in Z^{*}$ for any $n \in \mathbb{N}$. Then, we can prove the following lemma.

\section{Lemma 4.1.7}

1. $\left\{e_{n}\right\}$ is a 1 -unconditional basic sequence of $Z^{*}$.

2. If $X(\omega)$ is the closed linear span of $\left\{e_{n}\right\}$, then $X(\omega)^{*} \equiv Z$ and

$$
\mathbb{B}_{X(\omega)}=\overline{\operatorname{co}} E
$$

where $\mathbb{B}_{X(\omega)}$ is the closed unit ball of the space $X(\omega)$, $\overline{\text { co }}$ denotes the closure of the convex hull, and

$$
E=\left\{\theta_{m}\left(1-\omega_{m}\right) e_{m}+\sum_{i=1}^{n} \theta_{i} \omega_{i} e_{i}: m, n \in \mathbb{N},\left|\theta_{i}\right|=1, \forall i\right\}
$$


3. The basis $\left\{e_{n}\right\}$ is normalized.

We can find the proof of this lemma, as well as the proofs of the following results, in [Aco99a].

Now, we can see that the unit ball of the space $X(\omega)$ has no extreme points. Remember that, in Lindestrauss' construction, we showed that this point was essential and it was the reason for choosing $c_{0}$ as the domain space in his counterexample.

\section{Lemma 4.1.8}

1. $B_{X(\omega)}=\left\{u \in X(\omega):\left\|\frac{u}{1-\omega}\right\|_{1} \leq 1\right\}+\left\{v \in X(\omega):\left\|\frac{v}{\omega}\right\|_{\infty} \leq 1\right\}$

2. If $x_{0} \in \mathbb{B}_{X(\omega)}$ and $N \in \mathbb{N}$, there is $n \geq N$ and $\delta>0$ so that $\left\|x_{0}+\lambda e_{n}\right\| \leq 1$ for $\lambda \in \mathbb{K}$, with $|\lambda| \leq \delta$

Finally, from the two previous lemmas, we can prove the following theorem:

Theorem 4.1.9 There exists a Banach space $X$ such that, for any strictly convex infinitedimensional Banach space $Y, \overline{N A(X, Y)} \neq L(X, Y)$. In fact, $X$ can be taken as $X(\omega)$ for any $\omega \in \ell_{2} \backslash \ell_{1}$.

The same result also holds in the complex case if $Y$ is $\mathbb{C}$-strictly convex.

Proof: Fix $\omega=\left\{\omega_{n}\right\} \in \ell_{2} \backslash \ell_{1}$ such that $\left\{\omega_{n}\right\}$ is a decreasing sequence of positive real numbers and $\omega_{1}<1$. Then, by the Dvoretzky-Rogers theorem, which can be found, for instance, in [DJT95, theorem 1.2], there is a sequence $\left\{y_{n}\right\}$ of normalized vectors in $Y$ such that the series $\sum \omega_{n} y_{n}$ converges unconditionally ${ }^{1}$, so the set

$$
\left\{\sum_{n=1}^{\infty} \theta_{n} \omega_{n} y_{n}: \theta_{n} \in \mathbb{K},\left|\theta_{n}\right| \leq 1 \forall n \in \mathbb{N}\right\}
$$

is bounded [DJT95, theorem 1.9].

We want to prove that for any space $Y$ with the conditions above, we have $\overline{N A(X(\omega), Y)} \neq$ $L(X(\omega), Y)$. For that, we are going to construct an operator $T \in L(X(\omega), Y)$ such that $T \notin$ $\overline{N A(X(\omega), Y)}$.

Define $T: c_{00} \rightarrow Y$, given by

$$
T\left(\sum_{k=1}^{n} x(k) e_{k}\right)=\sum_{k=1}^{n} x(k) y_{k} .
$$

Let us check that $T$ is bounded, considering $c_{00} \subset X(\omega)$. By lemma 4.1.2, it is enough to show that $T$ is bounded in the subset $E$ of $\mathbb{B}_{X(\omega)}$, defined in the same lemma. Taking $x \in E$, it has the form

$$
x=\theta_{m}\left(1-\omega_{m}\right) e_{m}+\sum_{i=1}^{n} \theta_{i} \omega_{i} e_{i} \quad\left(\left|\theta_{i}\right|=1, m, n \in \mathbb{N}\right)
$$

so

\footnotetext{
${ }^{1}$ We say that the series $\sum_{n} x_{n}$ converges unconditionally if the sequence $\left\{x_{n}\right\}$ is unconditionally summable, i.e., $\sum_{n} x_{\sigma(n)}$ converges, regardless of the permutation $\sigma$ of the indices.
} 


$$
\|T x\| \leq 1+\left\|\sum_{i=1}^{n} \theta_{i} \omega_{i} y_{i}\right\| .
$$

As we saw previously, the series converges unconditionally. Then, $T$ is bounded in $E$. We still denote $T$ its continuous extension to $X(\omega)$. We will check that $T \notin \overline{N A(X(\omega), Y)}$.

Suppose that $S \in N A(X(\omega), Y)$. Then, $S$ attains its norm at a point $x \in \mathbb{B}_{X(\omega)}$, so, by lemma 4.1.8, there exists $n \in \mathbb{N}$ and $\delta>0$ such that

$$
\left\|x+\lambda e_{n}\right\| \leq 1, \quad|\lambda| \leq \delta .
$$

Therefore,

$$
\left\|S x+\lambda S e_{n}\right\| \leq\|S\|=\|S x\|, \quad|\lambda| \leq \delta .
$$

As $Y$ is strictly convex, or $\mathbb{C}$-strictly convex, $S e_{n}=0$. Then, since the basis $\left\{e_{n}\right\}$ is normalized, we have

$$
\|T-S\| \geq\left\|T e_{n}-S e_{n}\right\|=\left\|y_{n}\right\|=1,
$$

so $T$ cannot be approximated by norm-attaining operators.

R The operator $T$ defined in the proof above is never compact (since the set $\left\{y_{n}\right\}$ is not a relatively compact set). Therefore, this example will not constitute a counterexample for the case of norm-attaining compact operators, which will study later.

As a consequence of this theorem, we have some new examples of Banach spaces without property $B$.

- Example 4.1.10 The following Banach spaces are $\mathbb{C}$ - strictly convex. Then, none of them has property $B$.

1. Any complex infinite-dimensional space $L^{p}(\mu)(1 \leq p<\infty)$.

2. The Hardy space $H^{1}$. The Hardy space $H^{1}$ is the class of holomorphic functions $f$ on the open unit disk satisfying

$$
\sup _{0<r<1} \frac{1}{2 \pi} \int_{0}^{2 \pi}\left|f\left(r e^{i \theta}\right) d \theta\right|<\infty
$$

3. Any infinite-dimensional predual of a von Neumann algebra. A von Neumann algebra is a weakly closed $*$-algebra of bounded operators on a Hilbert space containing the identity.

Combining the results of Theorem 4.1.9 and Theorem 3.2.13, we have the following result:

Result 4.1.11 If $X(\omega)$ is defined as above, with $\omega \in \ell_{2} \backslash \ell_{1}$ and $Y$ is a strictly convex infinite dimensional Banach space (or $\mathbb{C}$-strictly convex), and we denote $Z=X \oplus_{\infty} Y$, then,

$$
\overline{N A(Z)} \neq L(Z)
$$


There is another result that appears in this paper and is worth mentioning. It is known that any Banach space can be renormed to verify property $B$ (see Theorem 3.1.20). However, by using renorming results, we can also see that almost any infinite-dimensional Banach space can be renormed without property $B$. An example of this is the following corollary of the previous theorem.

Corollary 4.1.12 Let $Y$ be a Banach space admitting a separable infinite-dimensional and complemented subspace. Then, $Y$ can be renormed without property $B$.

Proof: Let $M$ be a separable infinite-dimensional and complemented subspace of $Y$. Consider the projection

$$
P_{M}: Y \rightarrow Y
$$

such that $M=P_{M}(Y)$. As we can see in [DGZ93, chp 2, th 2.6], denote by $|\cdot|$ a strictly convex norm on $M$ equivalent to the original norm $\|\cdot\|$ of $Y$. Then, renorm $Y$ with the norm defined by:

$$
\||y|\| \mid=\max \{|P(y)|,\|y-P(y)\|\} \quad(y \in Y) .
$$

By Theorem 4.1.9, there exists an operator $T \in L(X(\omega), M)$ that cannot be approximated by norm-attaining operators. We denote by $T^{\prime}: X(\omega) \rightarrow Y$ the operator given by $T^{\prime}(x)=T(x)$. Then, it is clear that $P T^{\prime}=T$, so $T^{\prime} \notin \overline{N A(X(\omega), Y)}$ (otherwise, if $S \in N A(X(\omega), Y)$ is close to $T^{\prime}$ and satisfies $\|(I-P) S\|<\|P S\|$, then $P S$ is close to $P T^{\prime}$, which is not possible).

The paper from which we have extracted the results of this section, [Aco99a], was published one year after [Agu98], where the author proved that a stricly convex Banach space containing a symmetric basic sequence which is not equivalent to the $\ell_{1}$-basis or a normalized sequence with an upper $p$-estimate does not have property $B$. He deduced then that no infinite-dimensional uniformly convex Banach space has property $B$. This is another counterexample based on Lindenstrauss' argument.

We do not show these results, as every uniformly convex space is strictly convex in particular, so the main results of the paper are contained in the ones shown above, and we think the definitions and tools used in the results we have exposed are easier to understand.

\subsection{Other COUNTEREXAMPLES}

In this section, we are going to show an example constructed by J. Johnson and J. Wolfe of a compact, metric space $K$ such that $\overline{N A\left(L^{1}[0,1], C(K)\right)} \neq L\left(L^{1}[0,1], C(K)\right)$. For that, we will use the results of Section 3.3.

W. Schachermayer proved in [Sch83b] that the couple $\left(L^{1}[0,1], C[0,1]\right)$ also verifies this. Johnson and Wolfe used in [JW82] this example as an inspiration for their own proof, even though they thought it was too extense and complicated (although the example of Schachermayer was published in 1983, it had been constructed a year before).

For the definition of the set $K$, first we consider $K_{0}$ as the subset of $L^{\infty}[0,1] \equiv L^{1}[0,1]^{*}$ of the functions 


$$
\sum_{i=1}^{n}\left(1-2^{-i}\right) \chi_{E_{i}}
$$

where $n \in \mathbb{N}, E_{i} \subseteq[0,1], \lambda\left(E_{i}\right)<1 / 2^{-i}$ for every $i=1,2, \ldots, n$ and $E_{i} \cap E_{j}=\emptyset$ if $i \neq j$.

Then, we define $K$ as the closure in the $\omega^{*}$ topology of $K_{0}$, i.e.,

$$
K={\overline{K_{0}}}^{\omega^{*}}
$$

which is a compact metric space, due to the $\omega^{*}$ metrizability of $\mathbb{B}_{L^{\infty}[0,1]}$ and the Banach-Alaoglú theorem. ${ }^{2}$

The essential property of this space $K$ is contained in the following result.

Theorem 4.2.1 Let $\psi: K \rightarrow L^{\infty}$ be $\omega^{*}$-continuous and suppose, for each $k \in K$, that $\|\psi(k)-k\|$ $\leq \frac{1}{2}$ and $\|\psi(k)\|_{\infty} \leq 1$. Then, for each $k \in K$, and each $n \geq 2$,

$$
\mu\left(\left\{\omega \in[0,1]: \psi(k)(\omega) \geq 1-2^{-n-2}\right\}\right) \leq 16 \cdot 2^{-n},
$$

where $\mu$ denotes the Lebesgue measure.

Now let us see the applications of this result. Consider $g \in L^{\infty}[0,1]$ such that $\|g\|=1$ and $\mu(\{\omega \in[0,1]:|g(\omega)|=\|g\|=1\})>0$. The set of the points $g$ satisfying these conditions is dense in the surface of the ball of $L^{\infty}$ and they lie arbitrarily close to $S$. However, they do not satisfy the distributional condition of the previous theorem, what suggests that, in general, it is impossible to move the points in $K$ around the unit ball of $L^{\infty}$ in a $\omega^{*}$-continuous manner, with no point moved more than norm distance $1 / 2$, by a map $\psi$ such that one of the many nearby points $g$ as above is in the range of $\psi$.

For the proof of the theorem we need the following technical lemma, whose proof can be found in [JW82, Lemma 1].

Lemma 4.2.2 Suppose that $k=\sum_{i=1}^{n}\left(1-2^{-i}\right) \chi_{E_{i}} \in K_{0}$ and let $\psi$ satisfy the conditions in the previous theorem. Then,

$$
\left\|\psi(k) \chi_{E_{i}}\right\|_{\infty} \leq 1-2^{-i-2}
$$

for each $i=1, \ldots, n$.

Using this lemma, we can easily prove Theorem 4.2.1.

Proof: (of Theorem 4.2.1) We will prove it by contradiction. Let us suppose that there exist $k \in K$ and a natural $n \geq 3$ such that

$$
\mu\left(\left\{t \in[0,1]: \psi(k)(t) \geq 1-2^{-n-2}\right\}\right)>\frac{16}{2^{n}} .
$$

Then, we can find $E \subseteq[0,1]$ of measure $\mu(E)=16 \cdot 2^{-n}$ such that

\footnotetext{
${ }^{2}$ The Banach-Alaoglú theorem states that given a space $X$ which is the dual space of a normed space the closed unit ball of $X$ is compact with the $\omega^{*}$ topology.
} 


$$
\int_{E} \psi(k) d \mu \geq \mu(E)\left(1-2^{-n-2}\right) \text {. }
$$

As $K_{0}$ is $\omega^{*}$-dense in $S$ (by its definition) and $\psi$ is $\omega^{*}$-continuous, we obtain for every $\varepsilon>0$ an element $k_{0} \in K_{0}$ such that

$$
\int_{E} \psi(k) d \mu \leq \int_{E} \psi\left(k_{0}\right) d \mu+\varepsilon
$$

Now, as $k_{0} \in K_{0}$, then $k_{0}$ can be written in the form

$$
k_{0}=\sum_{j=1}^{m}\left(1-2^{-j}\right) \chi_{E_{j}}
$$

with the sets $E_{j}$ being pairwise disjoint. If we write

$$
A=\left\{t \in[0,1]: \psi\left(k_{0}\right)(t) \geq 1-2^{-n}\right\},
$$

then, by Lemma 4.2.2, we have

$$
\mu\left(A \cap E_{i}\right)=0 \text { if } i<n-2 .
$$

Moreover, if $t \in A \backslash \bigcup_{i=1}^{m} E_{i}$, then

$$
\psi\left(k_{0}\right)(t)-k_{0}(t) \geq 1-\frac{1}{2^{n}}>\frac{1}{2} .
$$

And as $\left\|\psi\left(k_{0}\right)-k_{0}\right\| \leq 1 / 2$, we obtain $\mu\left(A \backslash \bigcup_{i=1}^{m} E_{i}\right)=0$. Therefore,

$$
\mu(A) \leq \sum_{i=n-2}^{m} \mu\left(E_{i}\right)<\sum_{i=n-2}^{\infty} 2^{-i} \leq \frac{8}{2^{n}}
$$

Finally, it is clear that $\mu(E \cap A) \leq \mu(A) \leq 2^{3-k}$, and we took $E$ such that $\mu(E)=2^{4-k}$, so $\mu\left(E \cap A^{c}\right) \geq 2^{3-k}$. Therefore, using the previous expressions in boxes,

$$
\begin{aligned}
\mu(E)\left(1-\frac{1}{2^{n+2}}\right) & \leq \int_{E} \psi(k) d \mu \\
& \leq \int_{E} \psi\left(k_{0}\right) d \mu+\varepsilon \\
& =\int_{E \cap A} \psi\left(k_{0}\right) d \mu+\int_{E \cap A^{c}} \psi\left(k_{0}\right) d \mu+\varepsilon \\
& \leq \mu(E \cap A)+\left(1-\frac{1}{2^{n}}\right) \mu\left(E \cap A^{c}\right)+\varepsilon \\
& =\mu(E)-\frac{1}{2^{n}} \mu\left(E \cap A^{c}\right)+\varepsilon \\
& \leq \mu(E)-\frac{8}{2^{2 n}}+\varepsilon \\
& =\mu(E)\left(1-\frac{1}{2^{n+1}}\right)+\varepsilon
\end{aligned}
$$

And from these inequalities we deduce that $1-1 / 2^{n+2} \leq 1-1 / 2^{n+1}$ (due to the fact that $\varepsilon$ is arbitrary), which is a contradiction. 
Now, from this theorem we obtain the announced result.

Corollary 4.2.3 Define $T_{0}: L^{1}[0,1] \rightarrow C(K)$ by

$$
T_{0} f(k)=\int_{0}^{1} f(\omega) k(\omega) d \omega \text { for } f \in L^{1}[0,1] \text { and } k \in K
$$

If $T: L^{1}[0,1] \rightarrow C(K)$ is a linear operator with $\left\|T-T_{0}\right\| \leq 1 / 2$ and $\|T\| \leq 1$, then $T$ is not norm-attaining.

Proof: Consider $T \in L\left(L^{1}[0,1], C(K)\right)$ satisfying $\|T\|=1$ and $\left\|T-T_{0}\right\| \leq 1 / 2$. Let $\psi \in$ $\omega^{*}\left(K, L^{\infty}[0,1]\right)$ be the function which represents $T$, in the sense exposed in Section 3.3.

Suppose that $T$ attains its norm. Then, by Proposition 3.3.2, there exist $k \in K$ and $E \subseteq[0,1]$ such that

$$
\mu(E)>0 \text { and }|\psi(k)(t)|=1, \forall t \in E .
$$

We can take $T_{0}$ as represented by $i$, the inclusion of $S$ in $L^{\infty}[0,1]$. Then, as $\left\|T-T_{0}\right\| \leq 1 / 2$, we have $\|\psi-i\| \leq 1 / 2$; in particular, $\|\psi(k)-k\| \leq 1 / 2$ for every $k \in K$. And also, as $\|T\|=$ $1 \Rightarrow\|\psi\|=1$.

Then, using Theorem 4.2.1, we may suppose, without loss of generality, that $\psi(k)(t)=$ $-1, \forall t \in E$. And as $\|\psi(k)-k\| \leq 1 / 2$, it is clear that

$$
|\psi(k)(t)-k(t)| \leq 1 / 2, \forall t \in E .
$$

This implies that $k(t) \leq-1 / 2 \forall t \in E$.

However, the set

$$
\left\{f \in L^{\infty}[0,1]: \int_{E} f d \mu \geq 0\right\}
$$

is $\omega^{*}$-closed in $L^{\infty}[0,1]$ and contains $K_{0}$, so it contains $k \in K$ (remember that ${\overline{K_{0}}}^{\omega^{*}}$ ), which is impossible, as $k(t) \leq-1 / 2 \forall t \in E$ and $\mu(E)>0$ (so $k$ does not verify the hypothesis of the previous set).

In particular, $N A\left(L^{1}[0,1], C(K)\right)$ is not dense in $L\left(L^{1}[0,1], C(K)\right)$. Moreover, from the previous corollary and Theorem 3.2.13, we have the following result.

Result 4.2.4 Let $K$ be a compact, metric space defined as above. If we consider $X=$ $L^{1}[0,1] \oplus_{\infty} C(K)$, then

$$
\overline{N A(X)} \neq L(X)
$$




\subsection{SUMMARY}

NEGATIVE RESULTS FOR $\overline{N A(X, Y)}=L(X, Y) \quad(\overline{X Y})$

\begin{tabular}{|c|c|c|c|}
\hline$X$ & $Y$ & RESULT & COMMENTARIES \\
\hline$G$ (Gowers' space) & $\begin{array}{c}\text { A strictly convex space } \\
\text { such that there exists a } \\
\text { non-compact operator from } G \text { to } Y\end{array}$ & Corollary 4.1.4 & \\
\hline Exists $X$ & $\begin{array}{c}\text { Every strictly convex space } \\
\text { (or } \mathbb{C} \text {-strictly convex space) } \\
\text { with infinite dimension }\end{array}$ & Theorem 4.1.9 & $\begin{array}{c}\text { In fact, } X \text { can be taken as } \\
X(\omega) \text {, defined in Section } 4.1 .2\end{array}$ \\
\hline$L^{1}[0,1]$ & $\begin{array}{l}C(K) \text {, with } K \text { a } \\
\text { compact metric space }\end{array}$ & Corollary 4.2 .3 & \\
\hline
\end{tabular}

NEGATIVE RESULTS FOR $\overline{N A(X)}=L(X) \quad(\bar{X})$

\begin{tabular}{|c|c|c|}
\hline$X$ & RESULT & COMMENTARIES \\
\hline \hline $\begin{array}{c}G \oplus_{\infty} Y, \\
\text { with } Y \text { a strictly convex space } \\
\text { such that there exists a } \\
\text { non-compact operator from } G \text { to } Y\end{array}$ & Result 4.1 .5 & \\
\hline $\begin{array}{c}X \oplus_{\infty} Y, \\
\text { with } Y \text { a strictly convex space } \\
\text { (or } \mathbb{C} \text {-strictly convex space) } \\
\text { with infinite dimension }\end{array}$ & Result 4.1 .11 & $\begin{array}{c}\text { In fact, } X \text { can be taken as } \\
\text { ( }) \text { defined in Section } 4.1 .2\end{array}$ \\
\hline $\begin{array}{c}L^{1}[0,1] \oplus_{\infty} C(K), \\
\text { with } K \text { a compact metric space }\end{array}$ & Result 4.2 .4 & \\
\hline
\end{tabular}



THE APPROXIMATION PROPERTY

PROPERTIES $A^{k}$ AND $B^{k}$

POSITIVE RESULTS FOR $A^{k}$

POSITIVE RESULTS FOR $B^{k}$

COMPACT NORM-ATTAINING OPERATORS AND

THE DUNFORD-PETTIS PROPERTY

SUMMARY

BOOKS

ARTICLES

\section{COMPACT OPERATORS}

The aim of this chapter is to study the set of compact norm-attaining operators between two Banach spaces and its density in the set of compact operators between those two spaces. This problem has its origin in the problem of density of the set of norm-attaining operators between two Banach spaces, although it constitutes an interesting field of study itself.

In the second chapter of this survey, we showed the first negative example for the problem of density of the set of norm-attaining operators between two Banach spaces. This example was discovered by Lindenstrauss, and its essential point was that the closure of the set of normattaining operators between the two spaces he proposed $\left(c_{0}\right.$ and a strictly convex renorming of it, $Y$ ) is contained in the set of compact ones, so if there exists a non-compact operator from $c_{0}$ to $Y$, it cannot be approximated by norm-attaining operators.

Later, in chapter 4 , we showed some counterexamples based in the same arguments that Lindenstrauss', and all of them had in common that the technique used to prove the non-density of the set of norm-attaining operators was to find a non-compact operator which could not be a limit of norm-attaining operators.

Therefore, a natural question to ask is whether every compact operator between two Banach spaces can be approximated by norm-attaining operators. This question has been explicitly asked by Diestel and Uhl in [DU76], Johnson and Wolfe in [JW79] and, more recently, by M. Acosta in [Aco06]. In the past year, this question was answered negatively by M. Martín, in [Mar14], providing two Banach spaces and a compact operator between them which cannot be approximated by norm-attaining operators. This is one of the things we will show in this chapter.

In the first section of this chapter, we will introduce the approximation property and some properties and results about it. We will show many equivalent formulations of this property and use them to prove some results on norm-attaining compact operators. Among many other results, we will see the result by M. Martín mentioned above.

In the second section, we will define properties $A^{k}$ and $B^{k}$ for norm-attaining compact operators analogously as we did when we introduced properties $A$ and $B$ for norm-attaining operators. Later, we will show positive and negative examples of spaces verifying these properties. We will see that although many of these examples appear when we study properties $A$ and $B$ (even though we do not know whether properties $A$ and $B$ imply $A^{k}$ and $B^{k}$, respectively), there 
exist several results which are specific for properties $A^{k}$ or $B^{k}$ and not related to properties $A$ or $B$, respectively.

Finally, in the last section, we will introduce the Dunford-Pettis property and expose some results relating this property with the density of norm-attaining compact operators.

A result which goes back to the beginnings of functional analysis asserts that the compact operators on a Hilbert space are exactly those operators which are limits in norm of operators of finite rank. For Banach spaces, one part of this assertion is always true, i.e., if $X$ and $Y$ are Banach spaces and $T \in L(X, Y)$ verifies that there exists $\left\{T_{n}\right\} \subseteq F(X, Y)$ such that $\left\|T-T_{n}\right\| \rightarrow 0$, then $T$ is compact.

The converse is also true for many examples of spaces $X$ and $Y$ besides Hilbert spaces. For example, if $Y$ has a Schauder basis $\left\{y_{n}\right\}$, then, for every $T \in K(X, Y)$ we have $\left\|T-P_{n} T\right\| \rightarrow 0$, where the $\left\{P_{n}\right\}$ are the projections associated to the basis $\left\{y_{n}\right\}$. The question whether the converse assertion is true for arbitrary Banach spaces was open for a long time, until P. Enflo solved it negatively in 1973. For studying more positive and negative examples of spaces verifying that the set of compact operators between them is the same as the set of operators which are limit in norm of operators of finite rank, we introduce in the following section the concept of approximation property and study some properties about it. Some of the results presented in the following section have been extracted from [LT96].

Before beginning with the first section of this chapter, we will introduce some notation.

Notation 5.1. We will denote the set of compact norm-attaining operators between two Banach space $X$ and $Y$ as $N A K(X, Y)$ (it is clear then that $N A K(X, Y)=N A(X, Y) \cap K(X, Y)$ ).

As we have mentioned above, in this chapter we will study the problem of determining for which Banach spaces $X$ and $Y$ the set $N A K(X, Y)$ is dense in $K(X, Y)$. Analogously as we did for property $(\overline{X Y})$, we will denote this property by

$$
\overline{N A K(X, Y)}=K(X, Y) \quad\left(\overline{X Y}^{K}\right),
$$

and following this line of thought, for the case of endomorphisms, we denote this property by

$$
\overline{N A K(X)}=K(X) \quad\left(\bar{X}^{K}\right) .
$$

\section{1 The Approximation Property}

In the second chapter of this survey we defined the approximation property, in 2.2.14. Remember that we say that a Banach space $X$ has the approximation property $(A P)$ if for every compact set $K \subset X$ and every $\varepsilon>0$ there exists an operator $T: X \rightarrow X$ of finite-rank such that

$$
\|T x-x\| \leq \varepsilon
$$

for every $x \in K$. We will see later that this is equivalent to the fact that every compact operator is a limit of finite-rank operators (the converse is always true).

Every Hilbert space has this property. However, there are Banach spaces which do not. The first counterexample was published in 1973 by Per Enflo [Enf73], but a lot of work in this area had been previously done by Grothendieck. 
Grothendieck's work on the theory of Banach spaces and continuous linear operators introduced the approximation property. In a long monograph, Grothendieck proved that if every Banach space had the AP, then every Banach space would have a Schauder basis. Grothendieck thus focused the attention of functional analysts on deciding whether every Banach space have the approximation property. In 1972, Per Enflo constructed a separable Banach space that lacks both the approximation property and a Schauder basis.

Later many other counterexamples were found. In 1981, A. Szankowski proved in [Sza81] that the space of bounded endomorphisms on $\ell_{2}$ does not have the approximation property. And also the spaces $\ell_{p}$, with $p \neq 2$, and $c_{0}$ have closed subspaces that do not have the approximation property.

Before studying the approximation property, we need to introduce two general facts: One concerns the structure of compact sets in a Banach space and the other gives a concrete representation of the dual of some spaces of operators.

Proposition 5.1.1 A closed subset $K$ of a Banach space $X$ is compact if, and only if, there is a sequence $\left\{x_{n}\right\}$ in $X$ such that $\left\|x_{n}\right\| \rightarrow 0$ and $K \subset \overline{\operatorname{conv}}\left\{x_{n}\right\}$.

Proof: It is easy to check that if $\left\|x_{n}\right\| \rightarrow 0$, then the set

$$
\left\{\sum_{n=1}^{\infty} \lambda_{n} x_{n}: \lambda_{n} \geq 0, \sum_{n=1}^{\infty} \lambda_{n} \leq 1\right\}
$$

is compact and coincides with $\overline{\operatorname{conv}}\left\{x_{n}\right\}$. For the other implication, let $K$ be compact, and let $\left\{x_{i, 1}\right\}_{i=1}^{n_{1}}$ be a finite set of elements of $X$ so that

$$
2 K \subset \bigcup_{i=1}^{n_{1}} \mathbb{B}\left(x_{i, 1}, 1 / 4\right) .
$$

Consider

$$
K_{2}=\bigcup_{i=1}^{n_{1}}\left\{\left(\mathbb{B}\left(x_{i, 1}, 1 / 4\right) \cap 2 K\right)-x_{i, 1}\right\} .
$$

Then, $K_{2}$ is a compact subset of $\mathbb{B}(0,1 / 4)$. Next, pick $\left\{x_{i, 2}\right\}_{i=1}^{n_{2}}$ in $\mathbb{B}(0,1 / 2)$ so that

$$
2 K_{2} \subset \bigcup_{i=1}^{n_{2}} \mathbb{B}\left(x_{i, 2}, 1 / 4^{2}\right),
$$

and put

$$
K_{3}=\bigcup_{i=1}^{n_{2}}\left\{\left(\mathbb{B}\left(x_{i, 2}, 1 / 4^{2}\right) \cap 2 K_{2}\right)-x_{i, 2}\right\} .
$$

We continue inductively the construction of $\left\{x_{i, j}\right\}_{i=1}^{n_{j}}$ for $j=1,2,3, \ldots$. Therefore, for every $x \in K$ there is an $1 \leq i_{1} \leq n_{1}$ so that $2 x-x_{i_{1}, 1} \in K_{2}$; hence, there is an $1 \leq i_{2} \leq n_{2}$ so that $4 x-2 x_{i_{1}, 1}-x_{i_{2}, 2} \in K_{3}$. In general,

$$
x-\left(x_{i_{1}, 1} / 2+x_{i_{2}, 2} / 2^{2}+\ldots+x_{i_{k}, k} / 2^{k}\right) \in 2^{-k} K_{k+1} .
$$

It follows that $x \in \overline{\operatorname{conv}}\left\{x_{i, j}: 1 \leq i \leq n_{j}, j=1,2, \ldots\right\}$. Since $\left\|x_{i, j}\right\| \leq 2 \cdot 4^{-j+1}$, for $j>1$ and every $i \leq n_{j}$, the assertion is proved. 
Proposition 5.1.2 Let $X$ and $Y$ be Banach spaces and consider on $L(X, Y)$ the topology $\tau$ of uniform convergence on compact sets in $X^{a}$. Then, the set of bounded linear functionals on $(L(X, Y), \tau)$ consits of all functionals $\varphi$ of the form

$$
\varphi(T)=\sum_{i=1}^{\infty} y_{i}^{*}\left(T x_{i}\right), \quad\left\{x_{i}\right\}_{i=1}^{\infty} \subset X, \quad\left\{y_{i}^{*}\right\}_{i=1}^{\infty} \subset Y^{*}, \quad \sum_{i=1}^{\infty}\left\|x_{i}\right\|\left\|y_{i}^{*}\right\|<\infty .
$$

${ }^{a}$ This is the locally convex topology generated by the seminorms of the form $\|T\|_{K}=\operatorname{Sup}\{\|T x\|, x \in K\}$, where $K$ ranges over the compact subsets of $X$.

Proof: Assume that $\varphi$ has such representation. Assume also that $x_{i} \neq 0$ for every $i$. Take $\left\{\eta_{i}\right\}_{i=1}^{\infty}$ a sequence of positive scalars tending to $\infty$ so that

$$
\sum_{i=1}^{\infty} \eta_{i}\left\|x_{i}\right\|\left\|y_{i}^{*}\right\|=C<\infty .
$$

Put $K=\left\{x_{i} /\left\|x_{i}\right\| \eta_{i}\right\}_{i=1}^{\infty} \cup\{0\}$. Then, $K$ is compact and

$$
|\varphi(T)| \leq \sum_{i=1}^{\infty} \eta_{i}\left\|x_{i}\right\|\left\|y_{i}^{*}\right\|\left\|T\left(x_{i} /\left\|x_{i}\right\| \eta_{i}\right)\right\| \leq C\|T\|_{K} .
$$

Conversely, assume that $\varphi$ is a bounded linear functional on $L(X, Y)$ so that $|\varphi(T)| \leq C\|T\|_{K}$ for some constant $C$ and some compact set $K \subset X$. By Proposition 5.1.1, we may assume without loss of generality that $K=\overline{\operatorname{conv}}\left\{x_{n}\right\}_{n=1}^{\infty}$, where $\left\|x_{n}\right\| \rightarrow 0$.

Let $S: L(X, Y) \rightarrow(Y \oplus Y \oplus \ldots)_{\infty}$ be defined by $S(T)=\left(T x_{1}, T x_{2}, \ldots\right)$. Since $|\varphi(T)| \leq$ $C\|S(T)\|$, it follows that there exists a linear functional $\varphi$ defined on the closure of $S L(X, Y)$ so that $\varphi(T)=\psi(S(T))$. By the Hahn-Banach Theorem, we may extend $\psi$ to a continuous linear functional on $(Y \oplus Y \oplus \ldots)_{i} n f t y$, i.e., to an element of $\left(Y^{*} \oplus Y^{*} \oplus \ldots\right)_{1}$. Therefore, there exists a sequence $\left\{y_{n}^{*}\right\}_{n=1}^{\infty} \subset Y^{*}$ so that $\sum_{n=1}^{\infty}\left\|y_{n}^{*}\right\|<\infty$ and $\varphi(T)=\sum_{n=1}^{\infty} y_{n}^{*} T\left(x_{n}\right)$.

The next theorem is due to Grothendieck and clarifies the relation between the approximation property and the question of approximating compact operators.

Theorem 5.1.3 Let $X$ be a Banach space. The following five assertions are equivalent.

(i) $X$ has the approximation property.

(ii) For every Banach space $Y, \overline{F(X, Y)}=L(X, Y)$.

(iii) For every Banach space $Y, \overline{F(Y, X)}=L(Y, X)$.

(iv) For every choice of $\left\{x_{n}\right\}_{n=1}^{\infty} \subset X,\left\{x_{n}^{*}\right\}_{n=1}^{\infty} \subset X^{*}$ such that $\sum_{n=1}^{\infty}\left\|x_{n}^{*}\right\|\left\|x_{n}\right\|<\infty$ and $\sum_{n=1}^{\infty} x_{n}^{*}(x) x_{n}=0$ for all $x \in X$, we have $\sum_{n=1}^{\infty} x_{n}^{*}\left(x_{n}\right)=0$.

(v) For every Banach space $Y$, every $T \in K(Y, X)$ and every $\varepsilon>0$ there exists $R \in F(Y, X)$ with $\|T-R\|<\varepsilon$.

Proof: First, the equivalence $(i) \Leftrightarrow(i v)$ is a consequence of Proposition 5.1.2. Indeed, (i) means that the identity operator is in the closure, with the topology $\tau$, of $F(X)$ in $L(X)$, and this happens if and only if every $\tau$ continuous linear functional $\varphi$ on $L(X)$ which vanishes on operators of rank 1 vanishes also on the identity operator, what (iv) means. 
It is clear that $(i i)$ or $(i i i)$, taking $X=Y$, imply $(i)$. Then, we shall show that $(i)$ implies $(i i)$ and (iii). Let $T \in L(Y, X)$. Then, for every compact set $K \subset Y$ the set $T(K)$ is compact in $X$ (because $T$ is continuous). Hence, given $\varepsilon>0$, we have by $(i)$ a finite rank operator $R$ on $X$ so that

$$
\|R T y-T y\|<\varepsilon \text { for } y \in K \text {. }
$$

Since $R T$ is of finite rank, we have proved (ii). Consider now $0 \neq T \in L(X, Y)$ and let $K$ be a compact set in $X$ and $\varepsilon>0$. By $(i)$, there exists a finite rank operator $R$ on $X$ so that

$$
\|R x-x\|<\varepsilon /\|T\| \text { for } x \in K .
$$

Then, $\|T R x-T x\| \leq\|T\|\|R x-x\|<\varepsilon$ for $x \in K$ and this proves (iii).

It remains to prove the equivalence of $(i)$ and $(v)$. Assume that $(i)$ holds and let $T \in K(X, Y)$. Then, the set $K=\overline{T\left(\mathbb{B}_{Y}(0,1)\right)}$ is compact and hence, for every $\varepsilon>0$, there exists a finite rank operator $R$ on $X$ such that

$$
\|R x-x\|<\varepsilon /\|T\| \text { for } x \in K \text {. }
$$

Therefore, $\|R T-T\| \leq \varepsilon$ and, thus, $(v)$ holds.

Assume now that $(v)$ holds. We want to prove $(i)$. For that, let $K$ be a compact subset of $X$ and $\varepsilon>0$. By Proposition 5.1.2, we may assume without loss of generality that $K=\overline{\operatorname{conv}}\left\{x_{n}\right\}_{n=1}^{\infty}$, with $\left\{x_{n}\right\}$ verifying $\left\|x_{n}\right\| \rightarrow 0$ and $\left\|x_{1}\right\| \leq 1$. Consider $U=\overline{\operatorname{conv}}\left\{ \pm x_{n} /\left\|x_{n}\right\|^{1 / 2}\right\}_{n=1}^{\infty}$. Clearly, $U$ is a compact convex set in $X$ which is symmetric with respect to the origin.

Let $Y$ be the linear span of $U$ in $X$, i.e., $Y=\bigcup_{n=1}^{\infty} n U$, and introduce in $Y$ a norm $\||\cdot|||$ which makes $U$ its unit ball, i.e.,

$$
\||y|\|=\inf \{\lambda>0: y / \lambda \in U\} .
$$

It is easy to show that $(Y,\||\cdot|\| \mid)$ is a Banach space (in particular, it is complete). The formal identity map from $Y$ to $X$ is compact; hence, by (v), there exist $\left\{y_{i}^{*}\right\}_{i=1}^{m} \subset Y^{*}$ and $\left\{u_{i}\right\}_{i=1}^{m} \subset X$ so that

$$
\left\|\sum_{i=1}^{m} y_{i}^{*}(x) u_{i}-x\right\|<\varepsilon / 2 \text { for every } x \in U .
$$

Therefore, $x \in K$. It is clear that even though the $\left\{y_{i}^{*}\right\}_{i=1}^{m}$ are continuous with respect to $|\|\cdot \mid\|$, they need not to be continuous with respecto to $\|\cdot\|$ (and, thus, they are not in general restrictions of elements of $X^{*}$ to $Y$ ). In order to conclude the proof, we only have to verify that given any $y^{*} \in Y^{*}$ and $\delta>0$ there exists a $x^{*} \in X^{*}$ such that

$$
\left|y^{*}(x)-x^{*}(x)\right|<\delta \text { for } x \in K,
$$

i.e., $\left|y^{*}\left(x_{n}\right)-x^{*}\left(x_{n}\right)\right|<\delta$ for every $n$. Observe that since $x_{n} /\left\|x_{n}\right\|^{1 / 2} \in U$, we have $\left\|\left|x_{n} \|\right| \leq\right.$ $\left\|x_{n}\right\|^{1 / 2}$ for every $n$ and, thus, $\left\||| x_{n}\right\| \mid \rightarrow 0$. Therefore, there exists $n_{0} \in \mathbb{N}$ such that for $n \geq n_{0}$, we have $\left|y^{*}\left(x_{n}\right)\right|<\delta / 2$.

Put $K_{0}=2 \delta^{-1} \overline{\operatorname{conv}}\left\{ \pm x_{n}\right\}_{n=n_{0}+1}^{\infty}$ (the closures in $\|\cdot\|$ and $\||\cdot|\|$ are the same). Consider

$$
F=\left\{x: x \in \operatorname{span}\left\{x_{n}\right\}_{n=1}^{n_{0}}, y^{*}(x)=1\right\} .
$$


Then, $F$ is closed with the topology of $\|\cdot\|, K_{0}$ is compact with the same topology and $K_{0} \cap F=\emptyset$. By the geometric version of the Hahn-Banach Theorem, there is a $\|\cdot\|$-closed hyperplane $\hat{F}$ in $X$ so that $F \subset \hat{F}$ and $\hat{F} \cap K_{0}=\emptyset$.

Let $x^{*} \in X^{*}$ be such that $\hat{F}=\left\{x: x^{*}(x)=1\right\}$. Then, $x^{*}\left(x_{n}\right)=y^{*}\left(x_{n}\right)$ for $n \leq n_{0}$ and $\left|x^{*}\left(x_{n}\right)\right|<\delta / 2$ for $n>n_{0}$. Consequently,

$$
\left|x^{*}\left(x_{n}\right)-y^{*}\left(x_{n}\right)\right|<\delta \text { for every } n,
$$

as desired.

From this theorem, it is easy to prove the following equivalence.

Theorem 5.1.4 Let $X$ be a Banach space. Then, $X^{*}$ has the approximation property if, and only if, for every Banach space $Y$, every $\varepsilon>0$ and every operator $T \in K(X, Y)$, there exists a finite rank operator $R$ such that $\|T-R\| \leq \varepsilon$.

The implication to the left follows easily from the preovious theorem and the definition of approximation property. The other implication needs proving that if we have a finite dimensional subspace $D$ of the bidual of a Banach space $X$, then there exists an operator from $D$ to $X$ such that the norm of the operator is as close to 1 as we want and the operator restricted to the intersection of $X$ and $D$ is the identity.

The relation between the properties appearing in the two previous theorems is clarified in the following result.

Theorem 5.1.5 a) Let $X$ be a Banach space. If $X^{*}$ has the AP, then $X$ has the AP. In particular, if $X$ is reflexive, then $X$ has the AP if, and only if, $X^{*}$ has the AP.

b) There exists a separable Banach space having a Schauder basis whose dual is separable but fails to have the AP.

Assertion (a) follows immediately from the equivalence $(i) \Leftrightarrow(i v)$ of Theorem 5.1.3. The proof of assertion (b) uses the fact that there is a Banach space which fails to have the AP and some results about Schauder basis.

And finally, we will introduce the following characterization of the approximation property, known to A. Grothendieck, found in [Gro55], which follows easily from the compact factorization of every compact operator through a closed subspace of $c_{0}$.

Lemma 5.1.6 - GROTHENDIECK.

A Banach space $Y$ has the approximation property if, and only if, $\overline{F(X, Y)}=K(X, Y)$ for every closed subspace $X$ of $c_{0}$.

A proof of the lemma can be found in [Jar81, Theorem 18.3.2].

After introducing some results concerning the approximation property, we can go back to the problem of approximating compact operators by norm-attaining ones, as there are many results of this field which can be proved from results concerning the approximation property. The results we are presenting now have been mainly extracted from [Mar14].

In the proof of the first theorem we are presenting, we will use this result of V. Klee, which can be found in [DGZ93, Chapter II, Theorem 2.6]. 
Theorem 5.1.7 If $X$ is a separable Banach space, then $X$ admits a strictly convex equivalent renorming.

Now we can enunciate and demonstrate the following theorem, which constitutes a negative answer to the question whether the expression $\overline{N A K(X, Y)}=K(X, Y)$ is true for all $X$ and $Y$ Banach spaces.

Theorem 5.1.8 There exist compact linear operators between Banach spaces which cannot be approximated by norm-attaining operators.

Proof: Consider $X$ a closed subspace of $c_{0}$ failing the approximation property (we have already discussed that it exists; see, for instance, Enflo's example). Then, by Theorem 5.1.5, part a), $X^{*}$ also fails the approximation property. And by Theorem 5.1.4, this means that there exists a Banach space $Y$ and $T \in K(X, Y)$ which cannot be approximated by finite-rank operators.

Now, considering the closure of $T(X)$, we may suppose that $Y$ is separable. And as the approximation property has isomorphic nature, we may suppose that $Y$ is strictly convex (Theorem 5.1.7). Therefore, by Lemma 4.1.1 (in the particular case of $X$ a subspace of $c_{0}$ ), $T$ cannot be approximated by norm-attaining operators.

Next, we would like to present some ways of obtaining examples of spaces verifying the hypothesis in the previous theorem. First, with respect to the domain space, it is clear that in the previous proof we have only used the fact that $X$ is a subspace of $c_{0}$ whose dual fails the approximation property. Then, we have:

Proposition 5.1.9 For every closed subspace $X$ of $c_{0}$ such that $X^{*}$ fails the approximation property, there exists a Banach space $Y$ such that $\overline{N A K(X, Y)} \neq K(X, Y)$.

In fact, we can use a result due to W. Johnson and G. Schechtman, which appears in [JO01], that states that there exists a closed subspace of $c_{0}$ with Schauder basis whose dual fails the approximation property. Then, as a corollary of the previous proposition we have:

Corollary 5.1.10 There exist a Banach space $X$ with Schauder basis and a Banach space $Y$ such that $\overline{N A K(X, Y)} \neq K(X, Y)$.

Now, dealing with the codomain space, the idea of the proof of the previous theorem can be modified to show that for every strictly convex Banach space $Y$ without the approximation property we can obtain examples of spaces verifying the hypothesis of the theorem.

Proposition 5.1.11 Let $Y$ be a strictly convex Banach space without the approximation property. Then, there exist a Banach space $X$ and a compact linear operator from $X$ into $Y$ which cannot be approximated by norm-attaining operators.

Proof: By Lemma 5.1.6, there exists a closed subspace $X$ of $c_{0}$ such that $\overline{F(X, Y)} \neq K(X, Y)$. And again by Lemma 4.1.1, we obtain $N A(X, Y) \subset F(X, Y)$. Therefore,

$$
\overline{N A K(X, Y)} \subset \overline{F(X, Y)} \neq K(X, Y),
$$


so there exists a compact operator from $X$ into $Y$ which cannot be approximated by norm-attaining operators.

It is interesting to compare the result above with the one by M. Acosta showed in Section 4.1.2, where we saw that there exists a Banach space $X$ such that for every infinite-dimensional strictly convex Banach space $Y$, there exists a non-compact operator which cannot be approximated by norm-attaining operators.

Next, we would like to give a result for subspaces of complex $L^{1}(\mu)$ spaces. First, we need to introduce the following concept.

\section{Definition 5.1.12 - COMPLEX STRICT CONVEXITY.}

A complex Banach space $Y$ is said to be complex strictly convex if for every $y \in Y$ with $\|y\|=1$ and $z \in Y$, the condition $\|y+\theta z\| \leq 1$ for every $\theta \in \mathbb{C}$ with $|\theta|=1$ implies $z=0$.

It is clear that strictly convex complex Banach spaces are complex strictly convex, but the converse is false (for instance, $L^{1}(\mu)$ spaces are complex strictly convex, proved in [Ist84], but they are not strictly convex).

Using a similar procedure to the one used in the proof of Lemma 4.1.1, it is easy to see that:

Proposition 5.1.13 If $X$ is a closed subspace of $c_{0}$ and $Y$ is a complex strictly convex Banach space, then

$$
N A(X, Y) \subseteq F(X, Y)
$$

Therefore, the following result follows with the same proof as that of Proposition 5.1.11.

Proposition 5.1.14 Let $\mu$ be a measure and let $Y$ be a closed subspace of the complex space $L^{1}(\mu)$ without the approximation property. Then, there exists a Banach space $X$ such that $\overline{N A K(X, Y)} \neq K(X, Y)$.

We do not know whether this result is also true in the real case. However, in [Aco99b], M. Acosta showed that there exists a Banach space $X$ such that for every measure $\mu$ with $L^{1}(\mu)$ being infinite-dimensional, there is a non-compact operator from $X$ into $L^{1}(\mu)$ which cannot be approximated by norm-attaining operators.

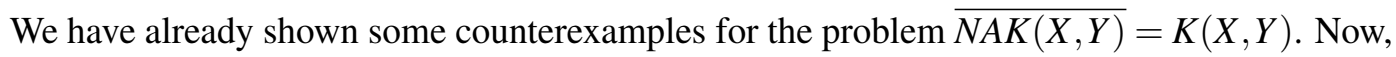
we are going to present some positive examples for it. For that, remember what we saw in Section 3.2: Every Banach space $X$ with the Radon-Nikodym property verifies that given a Banach space $Y$, every operator from $X$ to $Y$ can be approximated by compact perturbations of it attaining its norm. Therefore:

Proposition 5.1.15 Let $X$ be a Banach space with the Radon-Nikodym property. Then, for every Banach space $Y$,

$$
\overline{N A K(X, Y)}=K(X, Y)
$$


For spaces failing the Radon-Nikodym property, Diestel and Uhl showed in [DU76] that norm-attaining finite-rank operators from $L^{1}(\mu)$ into any Banach space are dense in the space of all compact operators. Johnson and Wolfe continued this study in [JW79], where they showed the same result for real $C(K)$ spaces. This result is proved using a stronger version of the approximation property of the dual. For the proof of the following results, we refer the reader to [Mar14].

Proposition 5.1.16 Let $X$ be a Banach space. Suppose that there is a net $\left\{P_{\alpha}\right\}$ of finite-rank contractive projections on $X$ such that for every $x^{*} \in X^{*},\left\{P_{\alpha}^{*} x^{*}\right\} \rightarrow x^{*}$ in norm. Then, for every Banach space $Y$,

$$
\overline{N A K(X, Y)}=K(X, Y) \text {. }
$$

Every $C(K)$ space satisfies the condition of the above proposition. And also as a consequence of this proposition, we have the following result.

Corollary 5.1.17 Let $X$ be a closed subspace of $c_{0}$ with a monotone Schauder basis. Then, for every Banach space $Y$,

$$
\overline{N A K(X, Y)}=K(X, Y) \text {. }
$$

\subsection{Properties $A^{k}$ AND $B^{k}$}

In this section, we will continue reviewing known results about density of norm-attaining compact operators. As this question is too general, we imitate what we did in Section 3.1, and introduce properties $A^{k}$ and $B^{k}$ for norm-attaining compact operators, analogously as we did in that section for norm-attaining operators. Most of the results of this section have been extracted from [Mar15].

Definition 5.2.1 - PROPERTY $A^{k}$.

Let $X$ be a Banach space. We say that $X$ has property $A^{k}$ if $\overline{N A K(X, Y)}=K(X, Y)$ for every Banach space $Y$.

\section{Definition 5.2.2 - PROPERTY $B^{k}$.}

Let $Y$ be a Banach space. We say that $Y$ has property $B^{k}$ if $\overline{N A K(X, Y)}=K(X, Y)$ for every Banach space $X$.

The first question we ask is whether properties $A$ and $B$ imply, respectively, properties $A^{k}$ and $B^{k}$. In fact, all sufficient conditions for properties $A$ and $B$ studied in this survey also imply, respectively, $A^{k}$ and $B^{k}$, as the usual way of establishing the density of norm-attaining operators is by proving that every operator can be approximated by compact perturbations of if attaining its norm.

Let us see now some examples of spaces without property $A^{k}$. 


\section{- Example 5.2.3}

- Every closed subspace of $c_{0}$ whose dual does not have the approximation property fails $A^{k}$. It is a consequence of Proposition 5.1.9.

- There exists a subspace of $c_{0}$ with Schauder basis failing property $A^{k}$. It is a consequence of Corollary 5.1.10.

Next, we would like to produce more examples of spaces without property $A^{k}$. For that, we need the following definition.

Definition 5.2.4 We say that the norm of a Banach space $X$ locally depends upon finitely many coordinates if for every $x \in X \backslash\{0\}$, there exist $\varepsilon>0$, a finite subset $\left\{f_{1}, f_{2}, \ldots, f_{n}\right\} \subseteq X^{*}$ and a continuous function $\varphi: \mathbb{R}^{n} \rightarrow \mathbb{R}$ such that $\|y\|=\varphi\left(f_{1}(y), f_{2}(y), \ldots, f_{n}(y)\right)$ for every $y \in X$ such that $\|x-y\|<\varepsilon$.

In [God01, Proposition III.3], it is proved that closed subspaces of $c_{0}$ have this property. And conversely, every infinite dimensional Banach space whose norm locally depends upon finitely many coordinates contains an isomorphic copy of $c_{0}$ (this result also appears in the same paper).

Now, with the definition we have just introduced, we are going to present more spaces without property $A^{k}$. For that, we need the following lemma, which is an extension of Lemma 4.1.1.

Lemma 5.2.5 Let $X$ be a Banach space whose norm locally depends upon finitely many coordinates and let $Y$ be a strictly convex Banach space. Then, $N A(X, Y) \subseteq F(X, Y)$.

Proof: Fix $x_{0} \in \mathbb{S}_{X}$. Then, as the norm of $X$ locally depends upon finitely many coordinates, there exist $\varepsilon>0,\left\{f_{1}, f_{2}, \ldots, f_{n}\right\} \subset X^{*}$ and a continuous function $\varphi: \mathbb{R}^{n} \rightarrow \mathbb{R}$ such that, for every $x \in X$ with $\left\|x_{0}-x\right\|<\varepsilon$,

$$
\|x\|=\varphi\left(f_{1}(x), f_{2}(x), \ldots, f_{n}(x)\right) .
$$

Consider $Z=\bigcap_{i=1}^{n} \operatorname{Ker} f_{i}$. Then, $Z$ has finite codimension. And for every $z \in Z$, with $\|z\|<\varepsilon$, it is clear that $\left\|\left(x_{0} \pm z\right)-x_{0}\right\|<\varepsilon$, so

$$
\left\|x_{0} \pm z\right\|=\varphi\left(f_{1}\left(x_{0} \pm z\right), f_{2}\left(x_{0} \pm z\right), \ldots, f_{n}\left(x_{0} \pm z\right)\right)=\varphi\left(f_{1}\left(x_{0}\right), f_{2}\left(x_{0}\right), \ldots, f_{n}\left(x_{0}\right)\right)=1,
$$

because $x_{0} \in \mathbb{S}_{X}$ and $\left\|x_{0}-x_{0}\right\|=0<\varepsilon$. Then, Lemma 4.1.1 gives the result.

Now, from this lemma, we can prove the following proposition, analogously as we did in Proposition 5.1.9.

Proposition 5.2.6 Let $X$ be a Banach space whose norm locally depends upon finitely many coordinates and whose dual fails the approximation property. Then, $X$ does not have property $A^{k}$. 
Proof: Consider $X$ verifying the previous hypothesis. Then, as $X^{*}$ fails the approximation property, there exists a Banach space $Y$ such that $\overline{F(X, Y)} \neq K(X, Y)$ (Theorem 5.1.4). Pick $T \in K(X, Y) \backslash \overline{F(X, Y)}$ and consider $Y$ as the range of $T$. Hence, by the hypothesis on $X, Y$ is separable, so it can be equivalently renormed to a strictly convex space. Thus, we may suppose that $Y$ is strictly convex.

Therefore, using the previous lemma,

$$
\overline{N A K(X, Y)} \subseteq \overline{N A(X, Y)} \subseteq \overline{F(X, Y)} \subsetneq K(X, Y),
$$

so $X$ does not have property $A^{k}$.

In fact, using this lemma we can also show some negative examples for property $A$.

Proposition 5.2.7 Let $X$ be an infinite dimensional Banach space whose norm locally depends upon finitely many coordinates. Then, $X$ does not have property $A$.

Proof: Let $Y$ be a strictly convex renorming of $X$. By the previous lemma, $N A(X, Y) \subseteq F(X, Y)$. As $X$ is infinite dimensional, the isomorphism from $X$ into $Y$ is non-compact. Therefore, it cannot be approximated by norm-attaining operators.

In particular, we get the following result for subspaces of $c_{0}$.

Corollary 5.2.8 If $X$ is an infinite dimensional closed subspace of $c_{0}, X$ does not have property $A$.

Returning to property $A^{k}$, as an application of Proposition 5.2.6, we get more examples of spaces failing property $A^{k}$. Before showing them, we have to introduce the following concept.

\section{Definition 5.2.9 - PolyHedRAL SPACE.}

A real Banach space is said to be polyhedral if the unit balls of all of its finite dimensional subspaces are polyhedra, i.e., the convex hull of finitely many points.

A typical example of polyhedral space is $c_{0}$ and, hence, its closed subspaces. For background on polyhedral spaces, consult [FLP01]. Every polyhedral Banach space admits an equivalent renorming which locally depends upon finitely many coordinates. Then, we obtain the following corollary of the previous proposition:

Corollary 5.2.10 Let $X$ be a polyhedral Banach space such that $X^{*}$ does not have the approximation property. Then, $X$ admits an equivalent renorming failing property $A^{k}$.

We have already presented some spaces failing property $A^{k}$. Now, we deal with the range space. Therefore, in the following results we will show some examples of Banach spaces failing property $B^{k}$.

Let us begin with a direct consequence of Grothendieck's Lemma. 
Proposition 5.2.11 Every strictly convex Banach space without the approximation property fails property $B^{k}$.

Proof: Let $Y$ be the strictly convex space without the AP. By Grothendieck's Lemma, 5.1.6, there exists a closed subspace $X$ of $c_{0}$ such that $\overline{F(X, Y)} \neq K(X, Y)$. And by Lemma 4.1.1, as we have already discussed several times in some previous results, $\overline{N A K(X, Y)} \neq K(X, Y)$, so $Y$ fails property $B^{k}$.

And we also have the following result, as a direct consequence of Proposition 5.1.14.

Proposition 5.2.12 Let $\mu$ be a measure and let $Y$ be a closed subspace of the complex space $L_{1}(\mu)$ without the approximation property. Then, $Y$ does not have property $B^{k}$.

\subsection{POSITIVe RESULtS FOR $A^{k}$}

In the previous pages, we have presented several negative examples for properties $A^{k}$ or $B^{k}$. Now, we are going to show some spaces verifying these properties.

Firstly, it is clear that every compact operator whose domain is reflexive attains its norm (because the unit ball of a reflexive space is $\omega$-compact). Therefore, every reflexive space has property $A^{k}$.

To get more positive examples for property $A^{k}$, we first recall that even though we still do not know whether property $A$ implies property $A^{k}$, the usual way to prove property $A$ for a Banach space $X$ is by showing that every operator from $X$ can be approximated by compact perturbations of it attaining its norm. Hence, the known examples of spaces with property $A$ actually have property $A^{k}$. In this line, as we have already seen that the main examples of spaces of this kind are spaces with the Radon-Nikodym property and those with property $\alpha$, we can see that both of these properties imply property $A$.

Proposition 5.2.13 The Radon-Nikodym property implies property $A^{k}$.

For the proof of this result, we refer the reader to [Ste86].

Proposition 5.2.14 Property $\alpha$ implies property $A^{k}$.

The proof of this result is completely analogous to the proof of Theorem 3.1.17.

By these two propositions, all the spaces presented in chapters 2 and 3 verifying either property $\alpha$ or RNP, have property $A^{k}$. Let us pass now to discuss on results which are specific of property $A^{k}$ and do not follow from property $A$. All results we know of this kind follow from the same general principle, a stronger version of the approximation property of the dual. As a consequence of Proposition 5.1.16, we have:

Proposition 5.2.15 Let $X$ be a Banach space. Suppose that there is a net $\left\{P_{\alpha}\right\}$ of finite-rank contractive projections on $X$ such that for every $x^{*} \in X^{*},\left\{P_{\alpha}^{*} x^{*}\right\} \rightarrow x^{*}$ in norm. Then, $X$ has 
property $A^{k}$.

From this result, we can obtain some examples of spaces with property $A^{k}$.

\section{- Example 5.2.16}

- For every locally compact Hausdorff space $L$, the space $C_{0}(L)$ has property $A^{k}$. The proof os this result can be easyly extended from the fact that real $C(K)$ spaces have the property given in the previous proposition.

- For every positive measure $\mu$, the space $L^{1}(\mu)$ has property $A^{k}$. This is shown in [DU76]. If the measure is finite, the above result also follows from the previous proposition. For the general case, a little bit more is needed.

- Every Banach space $X$ such that $X^{*}$ is isometrically isomorphic to $\ell_{1}$ has property $A^{k}$. This result can also be derived from the previous proposition.

- Every Banach space with a shrinking monotone Schauder ${ }^{1}$ basis has property $A^{k}$.

- Let $X$ be a Banach space with unconditional monotone Schauder basis which does not contain $\ell_{1}$. Then, $X$ has property $A^{k}$. This result is a direct consequence of the well-known fact that an unconditional Schauder basis of a Banach space is shrinking if the space does not contain $\ell_{1}$.

- Every $M$-embedded ${ }^{2}$ space with a monotone Schauder basis has property $A^{k}$.

- Every closed subspace of $c_{0}$ with monotone Schauder basis has property $A^{k}$. This comes from the fact that $c_{0}$ is a $M$-embedded space and $M$-embeddedness passes to closed subspaces.

We finish the positive examples for property $A^{k}$ by proving the following result, which constitutes a partial answer to the question whether either property $A$ of the AP of the dual are sufficient to get property $A^{k}$.

Proposition 5.2.17 Let $X$ be a Banach space having property $A$ and such that $X^{*}$ has the approximation property. Then, $X$ has property $A^{k}$.

Proof: As $X^{*}$ has the approximation property, $\overline{F(X, Y)}=K(X, Y)$ for every Banach space $Y$. And as we want to prove that $\overline{N A K(X, Y)}=K(X, Y)$ for every Banach space $Y$, we only need to prove that every finite-rank operator from $X$ can be approximated by compact norm-attaining operators.

Let $Y$ be an arbitrary Banach space and $T \in F(X, Y)$. Write $Z=T(X)$, which is finitedimensional. Then, every bounded linear operator is compact, something we discussed on the second chapter, so $N A(X, Z)=N A(X, Z) \cap K(X, Z)$ and $L(X, Z)=K(X, Z)$. And as $X$ has property $A$, we have $\overline{N A K(X, Z)}=K(X, Z)$. Therefore, we may find a sequence $\left\{T_{n}\right\} \subset$ $N A K(K, Z)$ converging to $T$, seen as an operator from $X$ into $Z$ (a finite-rank operator).

\footnotetext{
${ }^{1}$ A Schauder basis of a Banach space $X$ is said to be shrinking if its sequence of coordinate functionals is a Schauder basis of $X^{*}$.

${ }^{2} \mathrm{~A}$ Banach space $X$ is said to be $M$-embedded if $X^{\perp}$ is the kernel of an $L^{1}$-projection in $X^{*}$, i.e., $X^{*}=X^{\perp} \oplus Z$ for some $Z$ and $\left\|x^{\perp}+z\right\|=\left\|x^{\perp}\right\|+\|z\|$ for every $x^{\perp} \in X^{\perp}$ and $z \in Z$. For background, we refer the reader to [HWW93]
} 


\subsubsection{POSITIVE RESULTS FOR $B^{k}$}

Now, we are going to deal with the range space. In this subsection, we are going to present several examples of spaces verifying property $B^{k}$.

As we saw at the beginning of the survey, when we presented the classical Bishop-Phelps theorem, the first examples of a Banach space with property $B^{k}$ is the field (because we have already mentioned that when we place a finite dimensional space in the codomain, compact and bounded linear operators are the same).

To get more positive examples, and analogously to what we have done for property $A^{k}$, we start by recalling that even though it is not known whether property $B$ implies property $B^{k}$, the usual way to prove property $B$ for a Banach space $X$ is by showing that every operator into $Y$ can be approximated by compact perturbations of it attaining the norm.

This is what happens with property $\beta$, the main example of this kind. In Theorem 3.1.12, we showed that property $\beta$ implies property $B$. With a slight modification on the proof, we have the following result.

Proposition 5.2.18 Property $\beta$ implies property $B^{k}$.

We have also studied another sufficient condition for property $B$, the so-called property quasi- $\beta$. Again, the proof of the fact that property quasi- $\beta$ implies property $B$ can be adapted to the compact case.

Now, let us pass to discuss on results which are specific of property $B^{k}$ and not related to property $B$, analogously to what we did for property $A^{k}$. Most of the results we know of this kind follow from two general principles.

Proposition 5.2.19 Let $X$ be a Banach space with the approximation property. Suppose that for every finite dimensional subspace $Y$ of $X$ there exists a closed subspace $Z$ having property $B$ and such that $Y \subseteq Z \subseteq X$. Then, $X$ has property $B^{k}$.

This result applies to Banach spaces with the approximation property and satisfying that all its finite dimensional subspaces have property $B$, which is the case of the polyhedral spaces. Therefore, we have the following corollary.

Corollary 5.2.20 A polyhedral Banach space with the approximation property has property $B^{k}$.

In fact, we can extend this result to the complex case, as we observe that polyhedrality is equivalent to the fact that the norm of each finite dimensional subspace can be calculated as the maximum of the absolute value of finitely many functionals, and this implies property $\beta$ in the complex case.

Proposition 5.2.21 Let $X$ be a complex Banach space with the approximation property such that, for every finite dimensional subspace, the norm of any subspace of $X$ can be calculated as the maximum of the modulus of finitely many functionals. Then, $X$ has property $B^{k}$.

The second general principle for constructing results which are specific of property $B^{k}$ and 
not related to property $B$ is necessary due to the fact that we only know few examples of finite dimensional spaces with property $B$, and this is a limitation for Proposition 5.2.19. This second principle appeared in [JW79].

Proposition 5.2.22 A Banach space $X$ has property $B^{k}$ provided that there is a net of projections $\left\{Q_{\lambda}\right\}$ in $X$, with $\operatorname{Sup}_{\lambda}\left\|Q_{\lambda}\right\|<\infty$, such that $\left\{Q_{\lambda}(x)\right\} \rightarrow x$ in norm for every $x \in X$ and such that $Q_{\lambda}(X)$ has property $B^{k}$ for every $\lambda$.

We are going to show now some examples of spaces verifying property $B^{k}$, as a consequence of the results exposed above.

\section{- Example 5.2.23}

- Closed subspaces of $c_{0}$ (real or complex) with the approximation property have property $B^{k}$. This is a consequence of 5.2.21, because in [God01] the author proved that $c_{0}$ satisfies the condition of this proposition.

- Every predual of a real or complex $L^{1}(\mu)$ space has property $B^{k}$. This result was also shown by Johnson and Wolfe in the paper mentioned above and they proved it from Proposition 5.2.22.

- Real or complex $C_{0}(L)$ spaces have property $B^{k}$. This is a particular case of the previous one.

- For every positive measure $\mu$, the real space $L^{1}(\mu)$ has property $B^{k}$. This result is also a consequence of Proposition 5.2.19.

- Every uniform algebra ${ }^{3}$ has property $B^{k}$. This result appeared recently in [CGK13] and the proof is completely different to the previous ones in this chapter, as they do not use any kind of approximation property, but a nice complex version of Urysohn lemma constructed in the same paper.

\subsection{COMPACT NORM-ATtAINING OPERATORS AND tHE DUNFORD-PETTIS PROPERTY}

In the last section of the chapter on compact operators, we are going to study the relation between the density of compact norm-attaining operators and the Dunford-Pettis property. The results presented in this section have been mainly extracted from [Bak79].

In this section, we will study operators which attain their norm on $\mathbb{S}_{X^{* *}}$. We say that an operator $T \in L(X, Y)$ attains its norm on $\mathbb{S}_{X^{* *}}$ if $\|T\|=\left\|T^{* *} x^{* *}\right\|$ for some $x^{* *} \in X^{* *}$ with norm one. We also introduce now the following definition.

Definition 5.3.1 - StRICt DUNFORD-PettIS PROPERTY. A space $X$ is said to have the strict Dunford-Pettis property if for every Banach space $Y$ an arbitrary weakly compact operator $T \in L(X, Y)$ maps weakly Cauchy sequences to strongly Cauchy sequences.

With these definitions, and some elemental properties, we can prove the following result.

\footnotetext{
${ }^{3} \mathrm{~A}$ uniform algebra is a closed subalgebra of a complex $C(K)$ space that separates the points of $K$.
} 
Theorem 5.3.2 Let $X$ be a Banach space with $\mathbb{S}_{X^{* *}}$ sequentially compact in the $\omega^{*}$-topology of $X^{* *}$, i.e., the $\sigma\left(X^{* *}, X^{*}\right)$-topology. Then,

- If $T \in L(X, Y)$ is compact, $T$ attains its norm on $\mathbb{S}_{X^{* *}}$. Thus, every compact operator with reflexive domain $X$ attains its norm on $\mathbb{S}_{X}$.

- If $T \in L(X, Y)$ is weakly compact and $X$ has the strict Dunford-Pettis property, $T$ attains its norm on $\mathbb{S}_{X^{* *}}$. Therefore, if $Y$ is reflexive, every operator attains its norm on $\mathbb{S}_{X^{* *}}$.

Proof: We will only prove the second part of the theorem, as the proof for the first one is completely analogous. Consider a sequence $\left\{x_{n}\right\} \subseteq \mathbb{S}_{X}$ verifying $\|T\|<\left\|T x_{n}\right\|+1 / n$. Let $J$ be the canonical embedding of $X$ into $X^{* *}$. Since $\left\{J x_{n}\right\} \subseteq \mathbb{S}_{X^{* *}}$, there exists a subsequence (which we also call $\left\{x_{n}\right\}$, for simplicity), and a $x^{* *} \in \mathbb{S}_{X^{* *}}$ such that $\left\{J x_{n}\right\} \rightarrow x^{* *}$ in the $\sigma\left(X^{* *}, X^{*}\right)$-topology.

The sequence $\left\{x_{n}\right\}$ is weakly Cauchy in $X$, so, as $X$ has the strict Dunford-Pettis property, $\left\{T x_{n}\right\}$ is a Cauchy sequence in $Y$, then it is norm-convergent to some $y \in Y$. Since $\left\{J x_{n}\right\}$ is $\sigma\left(X^{* *}, X^{*}\right)$-convergent to $x^{* *}$ and $\left\{T x_{n}\right\}$ is norm-convergent (in particular weakly-convergent) to $y$, we have $T^{* *} x^{* *}=J y$.

Therefore,

$$
\left\|T x_{n}\right\| \rightarrow\|y\|=\left\|T^{* *} x^{* *}\right\|
$$

what implies that $\|T\|=\left\|T^{* *} x^{* *}\right\|$

A consequence of [Ste78, Theorem 3] is that if $\mathbb{S}_{X}$ has the RNP and we consider $\delta>0$, then every $T \in L(X, Y)$ may be written as $T=T_{1}+T_{2}$, where $T_{2}$ attains its norm on $\mathbb{S}_{X}$ and $T_{1}$ has rank one with $\left\|T_{1}\right\|<\delta$. A similar result can be proven from the previous theorem and [Lin63, Theorem 1].

Corollary 5.3.3 If $X$ is a Banach space with $\mathbb{S}_{X^{* *}} \sigma\left(X^{* *}, X^{*}\right)$-sequentially compact and $\delta>0$, every $T \in L(X, Y)$ may be written as $T=T_{1}+T_{2}$, where both attain their norm on $\mathbb{S}_{X^{* *}}$ and $T_{1}$ is compact with $\left\|T_{1}\right\|<\delta$.

Consider now $Y$ a weakly sequentially complete space and $X=c$, the space of convergent sequences. In [DS58, p. 515], the authors show that, in these conditions, every operator $T \in L(X, Y)$ is compact. Therefore, since $X=c, X^{*}$ is separable, so the first part of the previous theorem gives that every operator attains its norm on $\mathbb{S}_{X^{* *}}$, and the same can occur under the hypotheses of the second part.

Such cases render Theorem 1 of [Lin63] trivial, making it desirable to find useful subsets of norm-attaining operators which are dense in the operator space. An example of this situation is shown in [Bak79], where the author considers $X^{* *} / J(X)$ separable and $Y=\ell_{1}$ and uses the fact that, for such spaces, $L\left(X, \ell_{1}\right)$ consists entirely of compact operators [McW70, Theorem 5].

Let $X^{*} \otimes_{\lambda} Y$ denote the tensor product of $X^{*}$ and $Y$ equipped with the least crossnorm $\lambda$ [Sha50]. The assignment $\left(\sum f_{i} \otimes y_{i}\right)(x)=\sum f_{i}(x) y_{i}$ defines an isometric isomorphism of $X^{*} \otimes_{\lambda} Y$ onto the space $F(X, Y)$. Let $\left\{e_{i}\right\}$ be the usual unit vector basis of $\ell_{1}$ and consider

$$
P_{0}\left(X, \ell_{1}\right)=\left\{\sum_{i=1}^{n} f_{i} \otimes e_{i}: f_{i} \in X^{*}\right\}
$$


where $n$ is arbitrary and $f_{i}$ attains its norm on $\mathbb{S}_{X} . P_{0}\left(X, \ell_{1}\right)$ is not equivalent to the tensor product of two norm dense subsets. Then, the following result is verified.

Theorem 5.3.4 If $X^{* *} / J(X)$ is separable,

$$
\overline{P_{0}\left(X, \ell_{1}\right)}=L\left(X, \ell_{1}\right) \text {. }
$$

For the proof of this theorem, the author uses that if $X$ is a Banach space for which $X^{* *} / J(X)$ is separable, then $\mathbb{S}_{X^{* *}}$ is $\sigma\left(X^{* *}, X^{*}\right)$-sequentially compact. 


\subsection{SUMMARY}

$$
\text { Positive ReSULTS FOR } \overline{N A K(X, Y)}=K(X, Y) \quad\left(\overline{X Y}^{K}\right)
$$

\begin{tabular}{|c|c|c|c|}
\hline$X$ & $Y$ & RESULT & COMMENTARIES \\
\hline \hline $\begin{array}{c}\text { Radon-Nikodym } \\
\text { property }\end{array}$ & All & Proposition 5.1.15 & \\
\hline $\begin{array}{c}\text { There is a net }\left\{P_{\alpha}\right\} \text { of } \\
\text { finite-rank contractive projections } \\
\text { such that }\left\{P_{\alpha}^{*}\right\} \text { converges to Id } \\
\text { in the strong operator topology }\end{array}$ & All & Proposition 5.1.16 & All \\
\hline $\begin{array}{c}\text { Closed subspace of } c_{0} \\
\text { with a monotone Schauder basis }\end{array}$ & All & Definition 5.2.1 & This is a direct consequence \\
\hline of the previous result
\end{tabular}

$$
\text { Positive results for } \overline{N A K(X)}=K(X) \quad\left(\bar{X}^{K}\right)
$$

\begin{tabular}{|c|c|c|}
\hline$X$ & RESULT & COMMENTARIES \\
\hline \hline $\begin{array}{c}\text { Radon-Nikodym } \\
\text { property }\end{array}$ & Proposition 5.1.15 & \\
\hline $\begin{array}{c}\text { There is a net }\left\{P_{\alpha}\right\} \text { of } \\
\text { finite-rank contractive projections } \\
\text { such that }\left\{P_{\alpha}^{*}\right\} \text { converges to Id } \\
\text { in the strong operator topology }\end{array}$ & Proposition 5.1.16 \\
\hline $\begin{array}{c}\text { Closed subspace of } c_{0} \\
\text { with a monotone Schauder basis }\end{array}$ & Corollary 5.1.17 & $\begin{array}{c}\text { This is a direct consequence } \\
\text { of the previous result }\end{array}$ \\
\hline Property $A^{k}$ & Definition 5.2.1 & \\
\hline Property $B^{k}$ & Definition 5.2.2 & \\
\hline
\end{tabular}


EXAMPLES OF SPACES WITH PROPERTY $A^{k}$

( HENCE, POSITIVE RESULTS FOR $\left(\overline{X Y}^{K}\right)$ FOR EVERY $Y$ AND POSITIVE RESULTS FOR $\left(\bar{X}^{K}\right)$ )

\begin{tabular}{|c|c|}
\hline$X$ & RESULT \\
\hline $\begin{array}{l}\text { Radon-Nikodym } \\
\text { property }\end{array}$ & Proposition 5.2.13 \\
\hline Property $\alpha$ & Proposition 5.2.14 \\
\hline $\begin{array}{l}\text { There is a net }\left\{P_{\alpha}\right\} \text { of } \\
\text { finite-rank contractive projections } \\
\text { such that }\left\{P_{\alpha}^{*}\right\} \text { converges to Id } \\
\text { in the strong operator topology }\end{array}$ & Proposition 5.2.15 \\
\hline $\begin{array}{l}\qquad C_{0}(L) \text {, with } L \\
\text { a locally compact Hausdorff space }\end{array}$ & Example 5.2.16 \\
\hline $\begin{array}{l}L^{1}(\mu) \\
\text { with } \mu \text { a positive measure }\end{array}$ & Example 5.2.16 \\
\hline $\begin{array}{l}X \text { such that } X^{*} \\
\text { is isometrically isomorphic to } \ell_{1}\end{array}$ & Example 5.2.16 \\
\hline $\begin{array}{l}\text { Space with a shrinking } \\
\text { monotone Schauder basis }\end{array}$ & Example 5.2.16 \\
\hline $\begin{array}{l}\text { Space with unconditional monotone } \\
\text { Schauder basis which does not contain } \ell_{1}\end{array}$ & Example 5.2.16 \\
\hline $\begin{array}{l}M \text {-embedded space with a } \\
\text { monotone Schauder basis }\end{array}$ & Example 5.2.16 \\
\hline $\begin{array}{l}\text { Closed subspace of } c_{0} \text { with } \\
\text { monotone Schauder basis }\end{array}$ & Example 5.2.16 \\
\hline $\begin{array}{l}X \text { a space with property } A \\
\text { such that } X^{*} \text { has the AP }\end{array}$ & Proposition 5.2.17 \\
\hline
\end{tabular}




\section{EXAMPLES OF SPACES WITH PROPERTY $B^{k}$ ( HENCE, POSITIVE RESULTS FOR $\left(\overline{X Y}^{K}\right)$ FOR EVERY $X$ AND POSITIVE RESULTS FOR $\left(\bar{X}^{K}\right)$ )}

\begin{tabular}{|c|c|}
\hline$X$ & RESULT \\
\hline Property $\beta$ & Proposition 5.2.18 \\
\hline $\begin{array}{l}X \text { with the AP such that } \\
\text { for every finite dimensional subspace } \\
Y \text { of } X \text {, there exists a closed subspace } Z \\
\text { with property } B \text { such that } Y \subseteq Z \subseteq X\end{array}$ & Proposition 5.2.19 \\
\hline $\begin{array}{l}\text { Polyhedral space } \\
\text { with the approximation property }\end{array}$ & Corollary 5.2.20 \\
\hline $\begin{array}{l}X \text { a complex space with the AP such that } \\
\text { for every finite dimensional subspace } \\
\text { its norm can be calculated as the maximum } \\
\text { of the modulus of finitely many functionals }\end{array}$ & Proposition 5.2.21 \\
\hline $\begin{array}{c}\text { There is a net }\left\{Q_{\lambda}\right\} \text { of } \\
\text { projections with } \sup _{\lambda}\left\|Q_{\lambda}\right\|<\infty \\
\text { such that }\left\{Q_{\lambda}\right\} \rightarrow x \text { in norm for every } x \in X \\
\text { and } Q_{\lambda}(X) \text { has property } B^{k} \text { for every } \lambda\end{array}$ & Proposition 5.2.22 \\
\hline $\begin{array}{l}\text { (Real or complex) closed subspace of } c_{0} \\
\text { with the approximation property }\end{array}$ & Example 5.2.23 \\
\hline Predual of a (real or complex) $L^{1}(\mu)$ & Example 5.2.23 \\
\hline (Real or complex) $C_{0}(L)$ & Example 5.2.23 \\
\hline $\begin{array}{l}\text { The real space } L^{1}(\mu) \\
\text { for every positive measure } \mu\end{array}$ & Example 5.2.23 \\
\hline Uniform algebra & Example 5.2.23 \\
\hline
\end{tabular}


NEGATIVE RESULTS FOR $\overline{N A K(X, Y)}=K(X, Y) \quad\left(\overline{X Y}^{K}\right)$

\begin{tabular}{|c|c|c|c|}
\hline$X$ & $Y$ & RESULT & COMMENTARIES \\
\hline \hline $\begin{array}{c}\text { Closed subspace of } c_{0} \\
\text { such that } X^{*} \text { fails the AP }\end{array}$ & Exists $Y$ & Proposition 5.1.9 & $\begin{array}{c}\text { If } X \text { is a closed subspace of } c_{0} \\
\text { such that } X^{*} \text { fails the AP, there exists } Y \\
\text { such that } \overline{N A K(X, Y)} \neq K(X, Y)\end{array}$ \\
\hline $\begin{array}{c}\text { Exists } X \\
\text { with Schauder basis }\end{array}$ & Exists $Y$ & Corollary 5.1.10 & $\begin{array}{c}\text { There exist } X \text { with Schauder basis } \\
\text { and } Y \text { such that } \overline{N A K(X, Y)} \neq K(X, Y)\end{array}$ \\
\hline Exists $X$ & $\begin{array}{c}\text { Strictly convex space } \\
\text { without the AP }\end{array}$ & Proposition 5.1.11 & $\begin{array}{c}\text { If } Y \text { is a strictly convex space } \\
\text { without the AP, there exists } Y \\
\text { such that } \overline{N A K(X, Y)} \neq K(X, Y)\end{array}$ \\
\hline Exists $X$ & $\begin{array}{c}\text { Closed subspace of } \\
\text { complex } L^{1}(\mu) \text { without } \\
\text { the AP, with } \mu \text { a measure }\end{array}$ & Proposition 5.1.14 & $\begin{array}{c}\text { If } \mu \text { is a measure and } Y \text { is a } \\
\text { closed subspace of the complex } \\
L^{1}(\mu), \text { there exists } X \text { such that } \\
\overline{N A K(X, Y)} \neq K(X, Y)\end{array}$ \\
\hline
\end{tabular}

NEGATIVE RESULTS FOR $\overline{N A K(X)}=K(X) \quad\left(\bar{X}^{K}\right)$

\begin{tabular}{|c|c|c|}
\hline$X$ & RESULT & COMMENTARIES \\
\hline \hline $\begin{array}{c}X \oplus_{\infty} Y \\
\text { for any of the four cases } \\
\text { in the previous chart }\end{array}$ & Theorem 3.2.13 & $\begin{array}{c}\text { If } \overline{N A K(X, Y)} \neq K(X, Y), \\
\text { then } \overline{N A K\left(X \oplus_{\infty} Y\right)} \neq K\left(X \oplus_{\infty} Y\right)\end{array}$ \\
\hline
\end{tabular}





\section{BIBLIOGRAPHY}

\section{BOOKS}

[Agu95] F. J. Aguirre. Algunos problemas de optimización en dimensión infinita: Aplicaciones lineales y multilineales que alcanzan su norma. Universidad de Granada: Tesis Doctoral, 1995 (cited on pages 59, 60).

[Ban01] S. Banach. Theory of Linear Operations. American Mathematical Society, 2001 (cited on page 73).

[CG97] J. Castillo and M. González. Three-space Problems in Banach Space Theory. Springer Verlag, 1997 (cited on page 74).

[DGZ93] R. Deville, G. Godefroy, and V. Zizler. Smoothness and renormings in Banach spaces. New York: Longman: Pitman Monographs, Surveys in Pure, and Applied Mathematics, vol. 64, 1993 (cited on pages 93, 104).

[Die84] J. Diestel. Sequences and Series in Banach spaces. Springer Verlag: Graduate Texts in Mathematics, vol. 92, 1984 (cited on page 28).

[DJT95] J. Diestel, H. Jarchow, and A. Tonge. Absolutely summing operators. Cambridge University Press: Cambridge Studies in Advanced Mathematics, vol. 43, 1995 (cited on page 91).

[DU77] J. Diestel and J. Uhl. Vector Measures. Providence, Rhode Island: Amer. Math. Soc., Mathematical Surveys No. 15, 1977 (cited on pages 63-65, 71, 82).

[DS58] H. Dunford and J. T. Schwartz. Linear Operators I. N.Y.: Inter-Science Pub., 1958 (cited on page 114).

[DC01] J. Duoandikoetxea and D. Cruz-Uribe. Fourier analysis. American Mathematical Society, 2001 (cited on page 21).

[Háj+07] P. Hájek et al. Biorthogonal systems in Banach spaces. Springer Verlag: CMS Books in Mathematics. Canadian Mathematical Society, 2007 (cited on pages 54, 56).

[HWW93] P. Harmand, D. Werner, and D. Werner. M-ideals in Banach spaces and Banach algebras. Volume 1547. Springer-Verlag, Berlin: Lect. Notes in Math., 1993 (cited on page 111). 
[Hol75] R.B. Holmes. Geometric functional analysis and its applications. New York: Springer Verlag, 1975 (cited on page 58).

[Ist84] V.I. Istratescu. Strict Convexity and Complex Strict Convexity. Volume 89. Marcel Dekker, New York: Lect. Notes Pure Appl. Math., 1984 (cited on page 106).

[Jar81] H. Jarchow. Locally Convex Spaces. Teubner, Stuttgart, 1981 (cited on page 104).

[LT96] J. Lindenstrauss and L. Tzafriri. Classical Banach Spaces I. Springer Verlag, 1996 (cited on page 100).

[Pay93] R. Payá. PhD Course: Some optimization problems in Banach spaces. Normattaining operators. University of Granada, 1993 (cited on pages 53, 88, 89).

[Rui94] M. Ruiz-Galán. Operadores que alcanzan su norma. Universidad de Granada: Memoria de Licenciatura, 1994 (cited on pages 37, 79, 81).

[Sha50] R. Shatten. A theory of cross spaces. Volume 26. Annals of Math. Studies, 1950 (cited on page 114).

\section{ARTICLES}

[Aco99a] M.D. Acosta. "Denseness of norm-attaining operators into strictly convex spaces". In: Proc. Royal Soc. Edinburgh.129A (1999), pages 1107-1114 (cited on pages 90, 91, 93).

[Aco99b] M.D. Acosta. "Norm attaining operators into $L_{1}(\mu)$ ". In: Contemporary Mathematics.232 (1999), pages 1-11 (cited on page 106).

[Aco06] M.D. Acosta. "Denseness of norm attaining mappings". In: RACSAM.100 (2006), pages 9-30 (cited on page 99).

[AAP96] M.D. Acosta, F.J. Aguirre, and R. Payá. "A new sufficient condition for the denseness of norm attaining operators”. In: Rocky Mountain J. Math. 26 (1996), pages 407-418 (cited on pages 58-60).

[ABR03] M.D. Acosta, J. Becerra-Guerrero, and M. Ruiz-Galán. "Characterizations of the reflexive spaces in the spirit of James' Theorem”. In: Cont. Math.321 (2003), pages 1-14 (cited on page 76).

[Agu98] F. J. Aguirre. "Norm-attaining operators into strictly convex Banach spaces". In: J. Math. Anal. Appl.222 (1998), pages 431-437 (cited on pages 47, 93).

[Bak79] J.M. Baker. "A note on compact operators which attain their norm”. In: 82.2 (1979), pages 319-321 (cited on pages 113, 114).

[BP60] C. Bessaga and A. Pelczynski. "Banach spaces not isomorphic to their cartesian squares I”. In: Bull. Acad. Polon. Sci.8 (1960), pages 77-80 (cited on page 74).

[BP61] P. Bishop and R. Phelps. "A proof that every Banach space is subreflexive". In: Bull. Amer. Math. Soc.67 (1961), pages 97-98 (cited on page 19).

[BP63] P. Bishop and R. Phelps. "The support functionals of a convex set". In: Convexity, Proc. Symp. Pure Math., 7, Amer. Math. Soc. (1963), pages 27-35 (cited on page 19).

[Bol70] E. Bollobás. "An extension to the theorem of Bishop and Phelps". In: Bull. London Math. Soc.2 (1970), pages 181-182 (cited on page 19).

[Bou97] J. Bourgain. "On dentability and the Bishop-Phelps property". In: Israel J. Math., 28 (1997), pages 265-271 (cited on pages 63, 65, 71). 
[CGK13] B. Cascales, A.J. Guirao, and V. Kadets. "A Bishop-Phelps-Bollobás type theorem for uniform algebras”. In: Adv. Math.240 (2013), pages 370-382 (cited on page 113).

[CS08] Y. S. Choi and H.G. Song. "Property (quasi- $\alpha$ ) and the denseness of norm attaining operators". In: Math. Nachr., 281 (2008), pages 1264-1272 (cited on pages 60, 61, $63)$.

[Cla36] J. Clarkson. “Uniformly convex spaces". In: Trans. Amer. Math. Soc.,40 (1936), pages 396-414 (cited on page 31).

[DP74] W.J. Davis and R.R. Phelps. "The Radon-Nikodym Property and dentable sets in Banach spaces”. In: Proc. Amer. Math. Soc.45 (1974), pages 119-122 (cited on page 76).

[DU76] J. Diestel and J.J. Uhl. "The Radon-Nikodym theorem for Banach space valued measures". In: Rocky Mountain J. Math.6 (1976), pages 1-46 (cited on pages 99, 107, 111).

[Enf73] P. Enflo. "A counterexample to the approximation property in Banach spaces". In: Acta Math.130 (1973), pages 309-317 (cited on page 100).

[FLP01] V.P. Fonf, J. Lindenstrauss, and R. Phelps. "Infinite dimensional convexity". In: Handboook of the Geometry of Banach spaces.I (2001), pages 599-670 (cited on page 109).

[God01] G. Godefroy. "The Banach space $c_{0}$ ". In: Extracta Math.16 (2001), pages 1-25 (cited on pages 108, 113).

[GT93] B.V. Godun and S. Troyanski. "Renorming Banach spaces with fundamental biorthogonal systems". In: Contemp. Math.144 (1993), pages 119-126 (cited on page 54).

[Gow90] W. Gowers. "Symmetric block bases of sequences with large average growth". In: Israel J. Math.69 (1990), pages 129-149 (cited on page 88).

[GM97] W.T. Gowers and B. Maurey. "Banach spaces with small spaces of operators". In: Math. Annal.307 (1997), pages 543-568 (cited on page 75).

[Gro55] A. Grothendieck. "Produits tensoriels topologiques et espaces nucléaires". In: Mem. Amer. Math. Soc.16 (1955), 140 pp (cited on page 104).

[Hol73] J. R. Holub. "Reflexivity of $L(E, F)$ ". In: Proc. AMS.39 (1973), pages 175-177 (cited on page 28).

[Huf80] R. Huff. "On non-density of norm-attaining operators". In: Rev. Roum. Math. Pures et Appl.25 (1980), pages 239-241 (cited on pages 67, 71).

[JW79] J. Johnson and J. Wolfe. "Norm attaining operators". In: Studia Math.65 (1979), pages 7-19 (cited on pages 26, 90, 99, 107, 113).

[JW82] J. Johnson and J. Wolfe. "Norm attaining operators and simultaneously continuous retractions". In: Proc. Amer. Math. Soc.86 (1982), pages 609-612 (cited on pages 93,94$)$.

[JO01] W.B. Johnson and T. Oikhberg. "Separable lifting property and extensions of local reflexivity”. In: Illinois J. Math.45 (2001), pages 123-137 (cited on page 105).

[Kai95] K.E. Kaibkhanov. "On Banach spaces that are not isomorphic to their Cartesian squares”. In: Mat. Zametki.57 (1995), pages 534-541 (cited on page 75). 
[Lin63] J. Lindenstrauss. "On operators which attain their norm”. In: Israel J. Math 1 (1963), pages 139-148 (cited on pages 32, 34, 44, 47, 49, 50, 114).

[Mar14] M. Martín. “Norm-attaining compact operators”. In: J. Funct. Anal.267 (2014), pages 1585-1592 (cited on pages 67, 99, 104, 107).

[Mar15] M. Martín. "The version for compact operators of Lindenstrauss properties $A$ and B'. In: (to appear) RACSAM (2015) (cited on page 107).

[MMP06] M. Martín, J. Merí, and R. Payá. "On the intrinsic and the spatial numerical range”. In: J. Math. Anal. Appl.318 (2006), pages 175-189 (cited on page 28).

[McW70] R.D. McWilliams. "On certain Banach spaces which are $\omega *$-sequentially dense in their second duals". In: Duke J. Math.37 (1970), pages 121-126 (cited on page 114).

[Mor97] J.P. Moreno. "Geometry of Banach spaces with $(\alpha, \varepsilon)$-property or $(\beta, \varepsilon)$-property". In: Rocky Mountain, Journal of Mathematics.27.1 (1997), pages 241-256 (cited on page 52).

[Neg84] S. Negrepontis. "Banach spaces and Topology". In: Handbook of Set-Theoretic Topology (K. Kunen and J. Vaughan, Eds.), North-Holland, Amsterdam (1984), pages 1045-1142 (cited on page 58).

[Par82] J. Partington. "Norm attaining operators". In: Israel J. Math., 43 (1982), pages 273 276 (cited on page 51).

[Phe74] R. Phelps. "Dentability and extreme points in Banach spaces". In: J. Funct. Anal. 16 (1974), pages 78-90 (cited on page 69).

[Pli80] A.N. Plichko. "Constructions of bounded fundamental and total biorthogonal systems from unbounded systems". In: Dokl. Akad. Nauk USSR.254 (1980), pages 1923 (cited on page 56).

[PZ90] R. A. Poliquin and V. E. Zizler. "Optimization of convex functions on $\omega^{*}$-compact sets”. In: Manuscripta Math.68 (1990), pages 249-270 (cited on page 37).

[Ros69] H.P. Rosenthal. "On quasi-complemented subspaces of Banach spaces, with an appendix on compactness of operators from $L^{p}(\mu)$ to $L^{r}(v)$ ". In: J. Functional Analysis.4 (1969), pages 176-214 (cited on page 27).

[Sch83a] W. Schachermayer. "Norm attaining operators and renormings of Banach spaces". In: Israel J. Math., 44 (1983), pages 201-212 (cited on pages 50, 52, 53).

[Sch83b] W. Schachermayer. "Norm attaining operators on some classical Banach spaces". In: Pac. J. Math.105 (1983), pages 427-438 (cited on page 93).

[Sem60] Z. Semadeni. "Banach spaces non-isomorphic to their Cartesian square". In: Bull. Acad. Polon. Sci.8 (1960), pages 81-84 (cited on page 75).

[She85] S. Shelah. "Uncountable constructions for B.A. e. c. groups and Banach spaces". In: Israel J. Math.51 (1985), pages 273-297 (cited on page 57).

[Ste78] C. Stegall. "Optimization of functions on certain subsets of Banach spaces". In: Math. Ann.236 (1978), pages 171-176 (cited on pages 69, 114).

[Ste86] C. Stegall. "Optimization and differentiation in Banach spaces". In: Linear Alg. and Appl.84 (1986), pages 191-211 (cited on pages 69, 71, 110).

[Sza81] A. Szankowski. " $B(H)$ does not have the approximation property". In: Acta Math.147 (1981), pages 89-108 (cited on page 101).

[Sza86] S.J. Szarek. "A superreflexive Banach space which does not admit complex structure”. In: Proc. Amer. Math. Soc.97 (1986), pages 437-444 (cited on page 75). 
[Ziz73] V. Zizler. "On some extremal problems in Banach spaces". In: Math. Scand. 32 (1973), pages 214-224 (cited on page 34). 



\section{Index}

\section{A}

Approximation property

Atom .................................. 18

Atomic measure ................. 18

\section{B}

Biorthogonal system

.42

\section{C}

Complex strict convexity

\section{D}

Dentability

.52

\section{$\mathbf{F}$}

\section{Functional}

Norm-attaining $\ldots \ldots \ldots \ldots \ldots \ldots . \ldots$

Fundamental biorthogonal system

42

\section{L}

Long Schauder basis

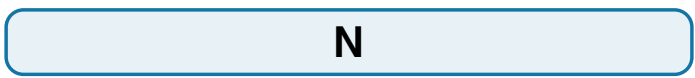

Norming set 48

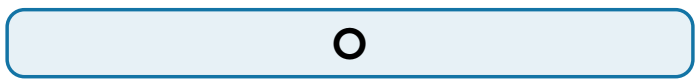

Operator

Compact.................... 17

Finite-rank .................... 18

Norm-attaining $\ldots \ldots \ldots \ldots \ldots \ldots 12$

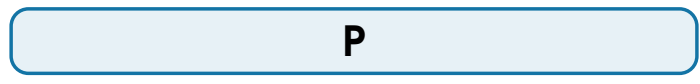

Property

$A^{k} \ldots \ldots \ldots \ldots \ldots \ldots \ldots \ldots 9 . \ldots 95$

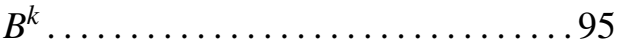

$\alpha \ldots \ldots \ldots \ldots \ldots \ldots \ldots \ldots, \ldots \ldots \ldots$

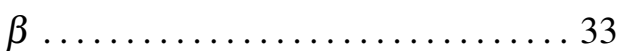

quasi $\alpha \ldots \ldots \ldots \ldots \ldots \ldots \ldots . \ldots 49$

quasi $-\beta \ldots \ldots \ldots \ldots \ldots \ldots \ldots . \ldots 46$

A ........................ 32

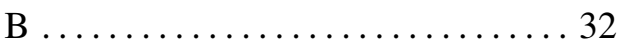

Bishop-Phelps.................52

Radon-Nikodym...............52

Strict Dunford-Pettis ............. 101 


\section{$\mathbf{R}$}

Rademacher system ................ 20

\section{S}

Shrinking Schauder basis ............ 98

Slice.........................56

Space

Gowers ..................... 72

James ...................... 62

M-embedded...................999

Polyhedral ....................... 97

Quasi-reflexive .................61 61

Reflexive .................... 10

Strongly exposed ................. 56

Support mappings ..................11

\section{T}

Theorem

Banach-Alaoglú ............... 26

Bishop-Phelps ................. 11

Bishop-Phelps-Bollobàs ........... 12

Bourgain....................53

Closed Graph ................. 15

Goldstine ................... 26

Hahn-Banach. .................8

Huff ........................ 55

James ..................... 10

Lindenstrauss-Zizler .............. 27

Partington.......................39

Radon-Nikodym................52

Riesz-Markov ................. 65

Schachermayer.............. 40

\section{U}

Uniformly strongly exposed points

W

Weakly compactly generated .40 